\title{
RDUS
}

Revue de DROIT UNIVERSITÉ DE SHERBROOKE

Titre : $\quad$ LE TRAITEMENT JURIDIQUE DU MINEUR SUICIDAIRE

Auteur(s) : $\quad$ Denise BOULET

Revue: $\quad$ RDUS, 2001-2002, volume 32, numéro 2

Pages: $\quad 317-426$

ISSN : $\quad 0317-9656$

Éditeur: $\quad$ Université de Sherbrooke. Faculté de droit.

URI : $\quad$ http://hdl.handle.net/11143/12328

DOI : https://doi.org/10.17118/11143/12328 
Page vide laissée intentionnellement. 
ARTICLE

\section{LE TRAITEMENT JURIDIQUE DU MINEUR SUICIDAIRE}

par Denise BOULET ${ }^{*}$

Au cours des dernières années, la fréquence du suicide chez les mineurs a grimpé d'une façon alarmante et ce, même s'ils sont pris en charge par le réseau de la santé et des services sociaux. Les moyens que s'est donnés le législateur pour protéger les jeunes suicidaires sont-ils adéquats? Les intervenants du réseau de la santé et des services sociaux savent-ils comment les mettre en oeuvre? Pour répondre à ces questions, l'auteure examine les trois avenues juridiques applicables aux mineurs suicidaires et démontre que les lois sont cohérentes et complémentaires. Par la suite, elle analyse le cadre d'intervention actuel et constate l'absence de concertation, l'incompréhension des lois et leur mauvaise application à l'intérieur du réseau médical et social. Par conséquent, la solution juridique au problème de l'incidence élevée du suicide ne réside pas dans des amendements législatifs. Comme voie de solution, l'auteure explique de quelle façon les établissements et les intervenants devraient appliquer les lois dans la gestion du risque suicidaire. Enfin, elle énonce quels éléments devraient être inclus dans les protocoles d'intervention qui permettraient de mieux coordonner les services, espérant ainsi pouvoir contribuer à diminuer le nombre de jeunes qui meurent par suicide au Québec.

In recent years, the incidence of suicide by minors has assumed alarming proportions even though these cases fall within the purview of the health and social services system. Have lawmakers put in place sufficient measures to adequately protect suicidal minors? Do health and social workers know how to properly carry them out? In answer to these questions, the writer examines three legal avenues applicable to suicidal minors and demonstrates that the laws in place are coherent and complementary. She then analyses the present framework for suicide intervention and points out a lack of concert as well as a misunderstanding and misapplication of legislation within the social and medical system itself. As a result, she concludes that a juridical solution to the problem of a high incidence of suicide among minors does not lie in legislative amendments. The writer indicates how both the system and its workers should apply existing law in the management of suicide risks. Finally, she brings out elements that should be included in all intervention plans in order to ensure better co-operation between services in the hope that this will contribute to decreasing the incidence of death by suicide of minors in Quebec.

*. $\quad$ Avocate. 


\section{SOMMAIRE}

INTRODUCTION

PARTIE PRÉLIMINAIRE : LES TROUBLES DE COMPORTEMENT CHEZ LE MINEUR . . . . . . 324

i) Un problème mixte $\ldots \ldots \ldots \ldots \ldots 324$

ii) Les établissements et leur mission . . . . 327

PARTIE I : LA PROTECTION DU MINEUR SUICIDAIRE: UNE RÉALITÉ LÉGISLATIVE BIEN

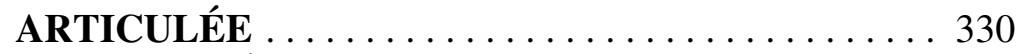

SECTION 1: LE RÉGIME DE DROIT COMMUN POUR

LES SOINS DE SANTÉ DES MINEURS _... . 330

A. Le consentement substitué ou le consentement personnel du mineur . . . . . . . . . . . . 334

i) L'effet de l'âge sur le consentement . . . 334

ii) La suprématie de principe de l'autorité parentale ................. 336

B. L'administration des soins de santé sans le consentement du mineur . . . . . . . . . . . . 340

i) L'intervention judiciaire $\ldots \ldots \ldots \ldots 340$

ii) Les cas d'urgence ............ 343

SECTION 2 : LES RÉGIMES D'EXCEPTION . . . . . . . . . 344

A. En vertu de la Loi sur la protection de la jeunesse ..................... 344

i) Les mesures d'urgence ......... . 349

ii) L'évaluation de la situation ........ 351

iii) L'intervention judiciaire ......... 353

iv) Les mesures applicables ......... 355

B. En vertu de Loi sur la protection des personnes dont l'état mental présente un danger pour ellesmêmes ou pour autrui . . . . . . . . . . 358 i) La notion de dangerosité $\ldots \ldots \ldots 359$ 
ii) La garde préventive et la garde provisoire ................ 362

iii) Les conditions d'obtention d'une ordonnance de garde en établissement . . 363

iv) Les effets de la garde en établissement . . 365

PARTIE II : POUR UNE MEILLEURE PROTECTION : L'UTILISATION DES RESSOURCES DANS LE RESPECT DES LOIS . . . . . . . . . . . . . . . . . . 368

SECTION 1: LA PRATIQUE : LE MYTHE DE LA PROTECTION .................... 369

A. Une analyse critique du cadre d'intervention actuel ....................... 371

i) La confusion dans les rôles $\ldots \ldots \ldots 372$

ii) La pratique en vase clos ........ 385

B. Une analyse critique des correctifs suggérés par le milieu . . . . . . . . . . . . . . . . 392

i) La position du Collège des médecins . . 392

ii) La position de l'Association des centres jeunesse du Québec . . . . . . . . . . . . 398

SECTION 2 : LA RÉCONCILIATION DES RÔLES . . . . . 404

A. Réapprendre sa mission ............. 404

i) Évaluer les besoins et orienter les personnes .................. 405

ii) Le dépistage précoce $\ldots . \ldots \ldots \ldots 409$

iii) La complémentarité à l'intérieur du réseau .................4 411

B. L'intervention étatique en dernier recours ..... 411

i) L'intervention du DPJ comme mesure d'exception . . . . . . . . . . . . . . . 412

- L'obligation de signaler . . . . . . 413

- La problématique de nature psychosociale ........... 415

ii) L'intervention du tribunal si nécessaire ..................... 417

- $\quad$ Pour les mineurs de moins de 14 ans ................ 419 
- $\quad$ Pour le mineur de 14 ans et plus

...............4 420

CONCLUSION ......................... 421 


\section{INTRODUCTION}

Les comportements dangereux des mineurs peuvent se manifester de plusieurs façons. Mentionnons, à titre d'exemples, l'agressivité, l'automutilation, la consommation excessive d'alcool ou de drogues, les idéations et les gestes suicidaires. Le phénomène du suicide chez les adolescents a récemment pris une place médiatique importante et des proportions inquiétantes au cours des dernières années. Ce serait même devenu la troisième cause de décès chez les moins de 20 ans. La clientèle des centres jeunesse constitue un groupe particulièrement à risque ${ }^{1}$.

Bien que des travaux de recherche aient permis de démontrer que des liens étroits existent entre certains désordres psychiatriques à l'adolescence et le suicide réussi ${ }^{2}$, la perspective est tout autre en ce qui a trait à l'étude des gestes autodestructeurs et des tentatives de suicide qui n'aboutissent pas au décès. En effet, suivant le docteur Patricia Garel ${ }^{3}$, «dans la majorité de ces cas, on ne peut identifier de pathologie psychiatrique aiguë» ${ }^{4}$. Ainsi, ces comportements n'appartiennent pas spécifiquement au champ de la psychiatrie et n'ont donc pas à être systématiquement pris en charge par le réseau de la santé. Ils peuvent être la manifestation d'une inadaptation relative et temporaire ne nécessitant aucun soin médical mais plutôt une intervention sociale ou psychologique qui, elle, relève a priori des services sociaux ${ }^{5}$.

Par ailleurs, une étude effectuée par le docteur Pierre Gagné, psychiatre, et publiée en 1994, ne laisse aucun doute, selon l'auteur, sur l'incidence élevée, qui serait de l'ordre de $90 \%$, de troubles mentaux chez les suicidés adolescents et adolescentes. Or, seulement 54 des 355 cas étudiés avaient été mis en contact

1. F. Maranda, «Désordres psychiatriques et suicide à l'adolescence» (1995) 5:4 Psychiatrie, recherche et intervention en santé mentale de l'enfant 382; Association des centres jeunesse du Québec, Le phénomène du suicide chez les jeunes; la prévention et l'intervention dans les centres jeunesse, novembre 1995 [non publié, archivé à l'Association des centres jeunesse du Québec à Montréal] à la p. 4.

2. Maranda, ibid.

3. P.Garel, «Comportements suicidaires à l'adolescence et intervention de crise» (1995) 5:4 Psychiatrie, recherche et intervention en santé mentale de l'enfant 412.

4. Ibid. à la p. 413.

5. $\quad$ Association des centres jeunesse du Québec, supra note 1 à la p. 19. 
avec des professionnels de la santé, six ont bénéficié d'un suivi soutenu et prolongé, et $0.5 \%$ d'un traitement pharmacologique ${ }^{6}$. Ces données amènent l'auteur à conclure que la majorité des adolescents qui se suicident au Québec sont «malades et la détresse psychologique qu'on peut observer chez eux dans les jours qui précèdent le décès apparaît être le reflet de la maladie plutôt que la cause elle-même du geste suicidaire» ${ }^{7}$. De plus, une enquête menée par un comité formé par l'Association des centres d'accueil du Québec en 1992 dans 35 centres de réadaptation a recensé, sur une période de deux ans, 289 tentatives de suicide parmi les jeunes qui y recevaient des services. Pendant ces deux années, huit autres jeunes se sont enlevé la vie ${ }^{8}$.

Sans être en mesure de prétendre, et encore moins de démontrer, que la mauvaise application de la législation est en partie responsable de ces constats alarmants, force nous est de relever que, depuis plusieurs années, les adolescents se suicident et ce, même s'il sont pris en charge par la société. Or, au Québec, trois avenues juridiques peuvent être empruntées pour tenter de protéger les mineurs ayant des problèmes de santé mentale de nature à mettre leur sécurité en danger et refusant les traitements qui leur sont proposés ${ }^{9}$. Ils peuvent être dirigés vers la Cour supérieure par le biais des dispositions du Code civil du Québec, vers la Direction de la protection de la jeunesse et pris en charge suivant la Loi sur la protection de la jeunesse ${ }^{10}$ et, enfin, leur cas peut être régi

6. P. Gagné, «Le suicide chez les moins de 20 ans» (1995) 5:4 Psychiatrie, recherche et intervention en santé mentale de l'enfant 375 à la p. 377.

7. Ibid. Plusieurs autres études permettent de soutenir que des liens étroits existent entre le suicide réussi à l'adolescence et des désordres psychiatriques. Voir notamment D. Brent, «Facteurs de risque associés au suicide à l'adolescence : revue des recherches» (1995) 5:4 Psychiatrie, recherche et intervention en santé mentale de l'enfant 360. Ainsi, d'autres auteurs sont d'avis que «le suicide dans nos sociétés occidentales est pratiquement toujours pathologique $[. .$.$] » et «on constate dans la majorité des cas la présence d'une maladie$ affective» : voir P. Lalonde et al., Psychiatrie clinique approche bio-psycho-sociale, Montréal, Gaëtan Morin, 1988 à la p. 599.

8. Y. Samson, Le phénomène du suicide chez les adolescents en centre de réadaptation, Commission des centres de réadaptation pour les jeunes en difficultés d'adaptation au Québec, 1992 [non publié, archivé à l'Association des centres d'accueil du Québec à Montréal] à la p. 5.

9. Pourrait également s'ajouter la Loi sur les jeunes contrevenants, L.R.C. 1985, c. Y-1 dont nous ne traiterons pas, n'ayant choisi d'aborder la question que sous l'aspect civil.

10. L.R.Q. c. P-34.1 [ci-après L.P.J.]. 
par le biais des dispositions de la Loi sur la protection des personnes dont l'état mental présente un danger pour elles-mêmes ou pour autrui ${ }^{11}$.

Eu égard à ce qui précède, il nous apparaît qu'orienter les adolescents atteints d'une maladie psychiatrique vers la Direction de la protection de la jeunesse est une erreur clinique avant d'être une erreur juridique et qu'elle peut s'avérer fatale, particulièrement si ces adolescents présentent un danger pour eux-mêmes. De la même façon, orienter vers les services de santé un adolescent qui manifeste une inadaptation relative et temporaire ne nécessitant aucun soin médical, mais plutôt une intervention psychosociale est également une erreur.

Il faut donc comprendre les lois en vigueur et voir comment elles doivent être appliquées afin d'être en mesure de diriger l'adolescent vers les portes du réseau susceptibles de répondre à la problématique en cause. Ceci permettra de distinguer les adolescents atteints d'une véritable pathologie psychiatrique des adolescents aux prises avec des problèmes psychosociaux, afin que les premiers aient accès aux soins de santé auxquels ils ont droit et que les seconds aient accès aux services sociaux requis par leur état.

Quel est l'intérêt d'une telle distinction? Quelle voie les intervenants du réseau devraient-ils privilégier et surtout, y a-t-il un intérêt thérapeutique ou juridique à distinguer? Nous croyons que cet intérêt est triple. Le premier, et le plus important, est de sauver des vies. Un adolescent ayant une pathologie de nature psychiatrique qui l'amène à avoir des idées suicidaires peut commettre des gestes autodestructeurs et en mourir, particulièrement s'il n'est pas vu par un médecin, donc non diagnostiqué et non traité. Or, la dépression, pour ne prendre que cet exemple, est une maladie qui peut frapper à tout âge et peut être traitée efficacement, entre autres, par médication. Non traitée, elle pourrait conduire au suicide. Le deuxième intérêt est de répondre aux besoins suivant la problématique en cause et de faire ainsi une utilisation intelligente des ressources de plus en plus rares dans le réseau. Le troisième intérêt est de respecter les autres droits des mineurs, particulièrement leur droit à l'intégrité

11. L.R.Q. c. P-38.001 [ci-après L.P.P.D.]. Suivant P.L. 39, $2^{\mathrm{e}}$ sess., $35^{\mathrm{e}}$ lég., Québec, 1997 (adopté le 17 décembre 1997, sanctionné le 18 décembre 1997, L.Q. 1997, c. 75 et entré en

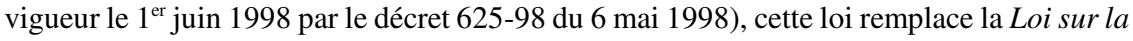
protection du malade mental, L.R.Q. c. P-41. 
et à l'inviolabilité de leur personne de même que leur droit à la liberté en ne leur imposant pas des hébergements en établissement qui seraient trop longs ou des interventions professionnelles non requises par leur situation.

Dans une partie préliminaire, nous exposerons comment les troubles de comportement pouvant conduire au suicide peuvent ouvrir deux portes dans notre réseau, soit celle des services de santé et celle des services sociaux. Par la suite, dans la première partie, nous ferons un survol théorique du régime de droit applicable aux mineurs, ce qui nous permettra, dans la deuxième partie, de cerner les difficultés qui se posent en pratique afin de faire ressortir les failles du système actuel. Pour ce faire, nous exposerons des exemples qui illustrent à quel point les dispositions législatives relatives aux soins de santé des mineurs sont mal comprises et surtout mal appliquées ${ }^{12}$. Enfin, dans la deuxième partie, nous cernerons les balises du cadre d'intervention applicable aux mineurs suicidaires à la lumière de la législation actuellement en vigueur. En conclusion, nous tenterons de faire des suggestions quant aux correctifs à apporter.

\section{PARTIE PRÉLIMINAIRE : LES TROUBLES DE COMPORTEMENT CHEZ LE MINEUR}

\section{i) Un problème mixte}

Une tentative de suicide est souvent le signe de graves difficultés sociales, interpersonnelles ou psychiatriques, telles la dépression ou le trouble du comportement ${ }^{13}$. Or, le trouble du comportement est une manifestation pouvant conduire à la fois à un diagnostic psychiatrique et à un motif

12. Nous avons effectué ces vérifications en partie alors que la L.P.P.D., ibid., n'était pas encore adoptée. Cependant, les nouvelles dispositions ne visent pas spécifiquement cette problématique et il y a tout lieu de croire qu'elles n'apporteront pas de changement significatif à la pratique déjà instaurée.

13. Classifiés respectivement sous les Troubles de l'humeur et le Trouble des conduites dans American Psychiatric Association, DSM-IV : Manuel diagnostique et statistique des troubles mentaux, trad. par J.-D. Guelfi et al., Paris, Masson 1996 (désigné par les praticiens et dans ce texte sous le sigle DSM-IV). Voir également Association des centres d'accueil du Québec, supra note 8; Association des centres jeunesse du Québec, supra note 1 à la p. 14 et $\mathrm{s}$. 
d'intervention suivant la Loi sur la protection de la jeunesse. En effet, la situation d'un adolescent suicidaire peut être signalée, évaluée et retenue en vertu de plusieurs alinéas des articles 38 et $38.1^{14}$. Ces articles stipulent que la sécurité ou le développement d'un enfant peut être compromis en l'absence de soins appropriés, s'il manifeste des troubles de comportement sérieux que les parents ne parviennent pas à contrôler ou s'il a besoin d'une prise en charge en établissement.

Par ailleurs, en psychiatrie, les praticiens utilisent le DSM IV ${ }^{15}$ qui représente le consensus d'experts et le résultat du regroupement d'un nombre considérable de données empiriques sur les critères diagnostiques et les formes cliniques de la maladie mentale : «[I]l est utilisé par des psychiatres, d'autres médecins, des psychologues, des travailleurs sociaux, des infirmières, des thérapeutes occupationnels et de réhabilitation, des conseillers et d'autres professionnels de la santé et de la santé mentale» ${ }^{16}$.

Parmi les Troubles habituellement diagnostiqués pendant la première enfance, la deuxième enfance ou l'adolescence, on y retrouve les Comportements perturbateurs, soit le «Trouble des conduites [sic] caractérisé par un ensemble de conduites dans lequel sont bafoués les droits fondamentaux d'autrui ou les normes et les règles sociales correspondant à l'âge du sujet ${ }^{17}$, le Trouble oppositionnel avec provocation, caractérisé par un ensemble de conduites négativistes, hostiles et provocatrices et, enfin, le Trouble comportement perturbateur non spécifié qui «s'applique aux troubles caractérisés par des conduites ou des comportements oppositionnels et provocateurs qui ne remplissent pas tous les critères» ${ }^{18}$ des deux premiers. De plus, le manuel comporte une section intitulée «Autres situations qui peuvent faire l'objet d'un examen clinique». Cette section traite des problèmes que peuvent présenter les enfants et les adolescents qui nécessitent une consultation mais qui ne sont pas définis comme des troubles mentaux, par exemple, les

14. L.P.J., supra note 10 . Voir également la partie I :, section 2 : «Les régimes d'exception», ci-dessous.

15. American Psychiatric Association, supra note 13.

16. Ibid. à la p. XXI.

17. Ibid. à la p. 102.

18. Ibid. à la p. 112. 
problèmes relationnels, les problèmes scolaires ou le comportement antisocial.

À première vue, ces troubles pourraient tous se qualifier sous la notion de troubles de comportement prévue à la Loi sur la protection de la jeunesse. L'absence de consultation médicale aura nécessairement pour effet qu'aucun diagnostic médical ne sera pas posé et, par voie de conséquence, la pathologie ne sera pas traitée. Dans certains cas, cette omission pourrait coûter la vie au jeune patient, particulièrement si ces difficultés cachent des idéations suicidaires ou des comportements potentiellement dangereux ${ }^{19}$.

En effet, le Trouble oppositionnel avec provocation peut survenir au cours d'un Trouble de l'humeur ${ }^{20}$ et avoir été précédé du Trouble des conduites. Les idées de suicide, les tentatives de suicide et les suicides surviennent à un taux relativement élevé chez les sujets ayant un Trouble des conduites et peuvent être associés à un ou plusieurs troubles mentaux dont les Troubles de l'humeur et le Trouble lié à une substance ${ }^{21}$. Parmi les Troubles de l'humeur, il y a notamment les Troubles dépressifs, le Trouble de l'humeur induit par une substance et le Trouble de l'humeur non spécifié, qui peuvent être précédés d'épisodes dépressifs majeurs au cours desquels les idées de mort, l'idéation suicidaire ou les tentatives de suicide sont fréquentes ${ }^{22}$. «La conséquence la plus

19. Consulter à ce sujet l'étude du docteur P. Gagné, supra note 6. À titre d'exemple, mentionnons le cas d'un adolescent décédé dans un centre de réadaptation à l'âge de 16 ans d'une asphyxie par pendaison qui a fait l'objet d'une enquête publique présidée par la Coroner Anne-Marie David dont le rapport a été rendu public le 18 novembre 1998. Au cours de l'enquête, la preuve a révélé que ce jeune présentait des comportements qui auraient pu permettre de poser le diagnostic de trouble des conduites ou de trouble de l'humeur et ce, plus d'une année avant son suicide. Suivant cette même preuve, les indices de dépression majeure nécessitant une consultation en psychiatrie ont commencé à se manifester plusieurs mois avant son suicide. Dans les jours précédents ce suicide, il connaissait un épisode de dépression majeure (page 32) nécessitant une consultation d'urgence en psychiatrie (page 44). Malgré deux tentatives de suicide dont une qualifiée de «sérieuse» par les intervenants, le jeune n'a pas vu de médecin et a réussi son troisième geste suicidaire deux jours plus tard. A.-M. David, «Rapport d'enquête publique sur le décès de Y. B.-L.», 27 octobre 1998 [non publié, archivé au Bureau du Coroner à Ste-Foy]; voir également la partie II :, section 1 : «La pratique : le mythe de la protection», ci-dessous.

20. American Psychiatric Association, supra note 13 à la p.109.

21. Ibid. à la p. 104.

22. Ibid. à la p. 379. Voir également Association des centres jeunesse du Québec, supra note 1 à la p. 14 et s.. 
grave d'un Épisode dépressif majeur est la tentative ou le suicide "réussi" ${ }^{23}$. Le Trouble dépressif majeur peut débuter à tout âge et atteindre les enfants prépubères de même que les adolescents ${ }^{24}$; il est associé à une mortalité élevée. En effet, jusqu'à $15 \%$ des sujets meurent de suicide ${ }^{25}$. La Dépendance à une substance peut contribuer au déclenchement ou à l'exacerbation de ce trouble ${ }^{26}$.

On voit donc que le problème est mixte et la solution pour réduire le taux alarmant de suicide chez les adolescents et adolescentes est certainement complexe. D'un point de vue purement légal, ce problème et sa solution ont pour toile de fond le rôle spécifique des intervenants œuvrant dans les établissements et appelés à intervenir auprès des jeunes qui peuvent présenter un risque suicidaire. Ce rôle est tributaire, en premier lieu, de la mission donnée aux établissements par le législateur dans la Loi sur les services de santé et les services sociaux ${ }^{27}$, tel que nous le verrons ci-après.

\section{ii) Les établissements et leur mission}

La mission des établissements de santé est élaborée à l'article 81 de la Loi sur les services de santé et les services sociaux ${ }^{28}$ qui se lit comme suit :

Art. 81 :

La mission d'un centre hospitalier est d'offrir des services diagnostiques et des soins médicaux généraux et spécialisés. À cette fin, l'établissement qui exploite un tel centre reçoit, principalement sur référence, les personnes qui requièrent de tels services ou de tels soins, s'assure que leurs besoins soient évalués et que les services requis, $y$ compris les soins infirmiers et les services psychosociaux spécialisés, préventifs ou de réadaptation, leur soient offerts à l'intérieur de ses installations ou, si nécessaire, s'assure qu'elles soient dirigées le plus

23. American Psychiatric Association, supra note 13 à la p. 380.

24. Ibid. à la p. 402.

25. Ibid. à la p. 401.

26. Ibid. à la p. 403.

27. L.R.Q. c. S-4.2 [ci-après L.S.S.S.S].

28. Ibid. 
tôt possible vers les centres, les organismes ou les personnes les plus aptes à leur venir en aide. [nos italiques.]

Cet article indique clairement que la mission d'un centre hospitalier est en premier lieu d'offrir des services diagnostiques et des soins médicaux. Face à un jeune patient suicidaire, l'établissement doit évaluer les besoins médicaux et cette évaluation doit nécessairement et exclusivement être faite par un médecin $^{29}$. Elle ne relève donc pas de la Direction de la protection de la jeunesse. Par la suite, l'établissement doit fournir, à l'intérieur de ses installations, les services requis, incluant les soins infirmiers et les services psychosociaux spécialisés. Ces services sont donc nécessairement accessoires aux services médicaux. S'il existe des personnes, des centres ou des organismes plus aptes à venir en aide au jeune, le centre hospitalier doit le diriger vers ces autres ressources. Pour orienter un adolescent qui manifeste des troubles de comportement ou qui verbalise des idées suicidaires vers une ressource qui n'est pas hospitalière, il faut donc que la problématique médicale soit éliminée ou, à tout le moins, qu'il ait été déterminé que cette problématique ne requiert pas une hospitalisation, mais simplement un suivi à l'externe. Dans ce cas, une collaboration et un suivi doivent être assurés pour que la prise en charge s'effectue de façon continue et personnalisée ${ }^{30}$.

L'adolescent aux prises avec des idées suicidaires ou manifestant des troubles de comportement pourrait faire l'objet d'un signalement à la direction de la protection de la jeunesse. Dans un tel cas, l'évaluation des besoins et les services offerts relèveraient du centre de protection de l'enfance et de la jeunesse $^{31}$. La mission de cet établissement est élaborée à l'article 82 de la Loi sur les services de santé et les services sociaux qui se lit comme suit :

29. Loi médicale, L.R.Q. c. M-9, art. 31, 43. Voir nos commentaires sous les notes 181 et 182, ci-dessous.

30. L.S.S.S.S., supra note 27, art. 5.

31. Ci-après C.P.E.J. 


\section{Art. 82 :}

La mission d'un centre de protection de l'enfance et de la jeunesse est d'offrir dans la région des services de nature psychosociale, y compris des services d'urgence sociale, requis par la situation d'un jeune en vertu de la Loi sur la protection de la jeunesse (chapitre P-34.1) et de la Loi sur les jeunes contrevenants (Lois révisées du Canada (1985), chapitre Y-1) ainsi qu'en matière de placement d'enfants, [...].

À cette fin, l'établissement qui exploite un tel centre s'assure que les besoins des personnes qui requièrent de tels services soient évalués et que les services requis par elles-mêmes ou par leur famille leur soient offerts soit directement, soit par les centres, les organismes ou les personnes les plus aptes à leur venir en aide. [nos italiques.]

Ainsi, la mission du C.P.E.J. est d'offrir des services de nature psychosociale, d'hébergement d'enfants et d'évaluation des besoins à cet égard. Les services diagnostiques, les soins médicaux et généralisés de même que l'évaluation des besoins relativement à la santé ne relèvent donc pas de la Direction de la protection de la jeunesse ni du C.P.E.J. Ces derniers n'ont pas à dispenser ces services qui sont, tel qu'indiqué, dans la mission exclusive des centres hospitaliers ${ }^{32}$. Ils doivent cependant s'assurer que ces services soient offerts.

Cette trame de fond ayant été élaborée, nous verrons comment le législateur a dressé, avec deux lois additionnelles ${ }^{33}$ et par des amendements au Code civil du Québec ${ }^{34}$, un système législatif complet de nature à répondre aux besoins des jeunes en détresse et qui peuvent mettre leur vie en danger en commettant un geste suicidaire.

32. $\quad$ L.S.S.S.S., supra note 27 , art. 81.

33. L.P.J., supra note 10; L.P.P.D., supra note 11.

34. Art. 28-33. 


\section{PARTIE I : LA PROTECTION DU MINEUR SUICIDAIRE: UNE RÉALITÉ LÉGISLATIVE BIEN ARTICULÉE}

Dans cette première partie, nous ferons un survol des dispositions législatives applicables à l'ensemble du réseau des services de santé et des services sociaux qui concernent la situation des mineurs suicidaires. D'abord, dans la première section, nous exposerons les règles de droit qui régissent la situation de tous les mineurs lorsqu'il est question de leur prodiguer des soins. Ensuite, dans la seconde section, nous exposerons deux lois d'exception et expliquerons dans quelles circonstances elles pourraient au besoin être appliquées, en sus du régime de droit commun, à la situation spécifique du mineur suicidaire.

\section{SECTION 1 : LE RÉGIME DE DROIT COMMUN POUR LES SOINS DE SANTÉ DES MINEURS}

$\mathrm{Au}$ Québec, le consentement aux soins de santé est régi par les dispositions du Code civil du Québec et ce, pour toute personne, quel que soit son âge ${ }^{35}$. La notion de santé, suivant le Code civil du Québec, couvre les aspects physique, mental, et psychologique ${ }^{36}$. Par conséquent, les soins de santé visent la réhabilitation de toute forme de pathologie pouvant donner ouverture à des idées ou à des gestes suicidaires ${ }^{37}$. La Loi sur les services de santé et les services sociaux élargit la notion de santé à l'aspect social ${ }^{38}$. Sauf dans les cas urgents $^{39}$, le droit civil exige que le consentement soit obtenu avant

35. É. Deleury et D. Goubau, Le droit des personnes physiques, $2^{\mathrm{e}}$ éd., Cowansville (Qc), Yvon Blais, 1997 aux pp. 5, 9, 23, 91 et s.

36. R. c. Morgentaler, [1988] 1 R.C.S. 30.

37. Pour une description et une classification de ces pathologies, voir la partie préliminaire «Les troubles de comportement chez le mineur», ci-dessus et American Psychiatric Association, supra note 13.

38. L.S.S.S.S., supra note 27, art. 1. De plus, suivant la définition donnée par l'Organisation mondiale de la santé, la notion de santé est «un état de complet bien-être physique, mental et social, [elle] ne consiste pas seulement en une absence de maladie ou d'infirmité» dans J. Hogart, Vocabulaire de la santé publique, Copenhague (Danemark), Organisation mondiale de la santé, Bureau régional de l'Europe, 1977 à la p. 208, cité dans P. LesageJarjoura, J. Lessard, et S. Philips-Nootens, Éléments de responsabilité civile médicale, Cowansville (Qc), Yvon Blais, 1995 à la p. 176, note 399.

39. Art. 13 C.c.Q. Voir la sous-section B. au point ii), «Les cas d'urgence», ci-dessous. 
l'administration de soins et ce, «quelle qu'en soit la nature, qu'il s'agisse d'examens, de prélèvements, de traitements ou de toute autre intervention ${ }^{40}$. Cette exigence repose sur l'article 10 C.c.Q. en vertu duquel «toute personne est inviolable et a droit à son intégrité». Ce principe, également reconnu dans la Charte des droits et libertés de la personne ${ }^{41}$, est fondamental ${ }^{42}$ et le législateur n'y tolère que peu d'exceptions ${ }^{43}$. Par conséquent, nul ne peut porter atteinte à la personne, même si elle est mineure et suicidaire, ni lui administrer des soins sans son consentement libre et éclairé, sauf dans les cas prévus par la loi ${ }^{44}$.

Pour être en mesure de donner un consentement libre et éclairé, la personne doit être capable et apte à le faire ${ }^{45}$. L'aptitude est la règle et est présumée dans notre droit ${ }^{46}$. Ainsi, quelle que soit la maladie dont souffre la personne, incluant la maladie mentale ${ }^{47}$, et quels que soient les symptômes

40. Art. 11 C.c.Q. Le Code de déontologie des médecins, R.R.Q. 1981, c. M-9, r.4, art. 2.03.28 énonce le même principe.

41. L.R.Q. c. C-12, art. 1; Lesage-Jarjoura, Lessard et Philips-Nootens, supra note 38 à la p. 131.

42. Ibid., Deleury et Goubau, supra note 35 à la p. 92.

43. La doctrine et la jurisprudence ont reconnu ce droit à l'autodétermination. Voir notamment A. Bernardot et R.P. Kouri, La responsabilité civile médicale, Sherbrooke (Qc), Éditions R.D.U.S., 1980 aux pp. 129-137; Lesage-Jarjoura, Lessard et Philips-Nootens, supra note 38 à la p. 131; A. Mayrand, L'inviolabilité de la personne humaine, Montréal, Wilson \& Lafleur, 1975 à la p. 15 et s.; Beausoleil c. La Communauté des Soeurs de la Charité de la Providence, [1965] B.R. 37 [ci-après Beausoleil]; Nancy B. c. Hôtel-Dieu de Québec, [1992] R.J.Q. 361 (C.S.) [ci-après Nancy B.]; Malette c. Shulman (1990), 72 O.R. (2 $) 417$ (1990), 37 O.A.C. 281 [ci-après Malette]; Manoir de la Pointe Bleue (1978) inc. c. Corbeil, [1992] R.J.Q. 712 (C.S.) [ci-après Manoir de la Pointe Bleue (1978) inc.].

44. Article 10 C.c.Q. Cette exception vise trois situations soit l'urgence, les cas où un consentement substitué peut être obtenu et les cas où une autorisation judiciaire est requise. Voir la section 2 :, sous-section A. aux points i) à iv), ci-dessous; Deleury et Goubau, supra note 35 aux pp. 94-95.

45. Deleury et Goubau, ibid. aux pp. 102-104, 332 . Sujet de droit à part entière, suivant l'article 1 C.c.Q., le mineur a la pleine jouissance de ses droits civils. Nous verrons cependant que son incapacité d'exercice, et donc la possibilité de donner seul un consentement aux soins de santé, est modulée en fonction de son âge : voir la section 1 :, sous-sectin A. aux points i) et ii), ci-dessous; Deleury et Goubau, ibid aux pp. 114-121.

46. $\quad$ Art. 4 et 154 C.c.Q. Deleury et Goubau, ibid. aux pp. 332-333.

47. Cité de la santé de Laval c. Lacombe, [1992] R.J.Q. 58 (C.S.) [ci-après Lacombe] et Institut Philippe Pinel de Montréal c. Blais, [1991] R.J.Q. 1969 (C.S.) [ci-après Blais]. 
manifestés par cette personne, incluant des attitudes violentes ou dangereuses ${ }^{48}$, elle sera présumée apte à consentir aux soins de santé qui la concernent. En cas de doute, le médecin doit évaluer, avant de $\operatorname{traiter}^{49}$, si la personne est effectivement apte.

Le Code civil du Québec n'élabore pas de critères permettant de déterminer si une personne est apte ou non à consentir à des soins. Dans une décision rendue en $1991^{50}$, la Cour supérieure a adopté un test en cinq volets, test mis de l'avant par la Nouvelle-Écosse, qui permet d'apprécier l'inaptitude à cet égard. Ce test a été repris en 1994 par la Cour d'appel et consiste à répondre aux questions suivantes :

1. Le patient réalise-t-il que le psychiatre l'examine pour déterminer sa capacité et comprend-il le sens de ce terme?

2. Le patient comprend-il la nature de la maladie pour laquelle on lui propose le traitement en question?

3. Le patient comprend-il la nature et le but du traitement?

4. Le patient saisit-il les risques et les avantages du traitement s'il le subit?

5. Le patient saisit-il les risques et les avantages du traitement s'il ne le subit pas? ${ }^{51}$

On voit donc que le but de ce test est de déterminer si le patient est en mesure de donner un consentement libre et éclairé aux traitements qui lui sont proposés ou de les refuser ${ }^{52}$. Ceci implique que «le patient doit être informé par son médecin de sa condition de façon à prendre une décision en pleine connaissance de cause; le patient doit être capable de recevoir et de comprendre

48. Institut Philippe Pinel de Montréal c. G.(A.), [1994] R.J.Q. 2523 (C.A.) [ci-après G.(A.)].

49. Ibid.; Deleury et Goubau, supra 35 à la p. 98.

50. Blais, supra note 47.

51. G.(A.), supra note 48 à la p. 2533.

52. Douglas Hospital Center c. C.T., [1993] R.J.Q. 1128 (C.S.) à la p. 1131 [ci-après C.T.]; Hôpital Charles-Lemoyne c. Forcier, [1992] R.D.F. 257 (C.S.) [ci-après Forcier]. 
l'information; et enfin, le patient doit être en mesure de prendre une décision et de l'exprimer ${ }^{53}$. En cas d'inaptitude de la personne, la loi autorise le recours à un consentement substitué qui peut être donné par le représentant, les proches parents ou une personne significative pour le patient inapte ${ }^{54}$.

En vertu de ces principes juridiques, à moins que la personne ne soit déclarée inapte, son refus d'être traitée doit être respecté ${ }^{55}$ car elle a le droit de décider de quelle façon elle souhaite disposer de son corps. Elle peut même prendre de mauvaises décisions ou des décisions qui peuvent apparaître déraisonnables. Par exemple, dans l'affaire bien connue de Nancy $B .{ }^{56}$, son refus de traitement fut respecté par le tribunal même s'il risquait d'entraîner sa mort et ce, malgré le fait que le tribunal n'était pas en accord avec ce choix. Également, dans l'affaire Malette c. Shulman, ${ }^{57}$ en Ontario, une action pour voies de fait contre un médecin fut accueillie parce qu'il avait donné une transfusion sanguine à sa patiente, témoin de Jéhovah, malgré une note écrite trouvée dans le porte-monnaie de celle-ci et indiquant spécifiquement qu'elle refusait toute transfusion sanguine.

Ces deux exemples, de même que les notions énoncées ci-dessus, concernent des personnes majeures. Nous verrons, dans les pages qui suivent, comment ces principes peuvent, en partie, s'appliquer aux mineurs.

53. Lacombe, supra note 47 à la p. 60. Voir également C.T. ibid.; Blais, supra note 47; Forcier, ibid.

54. $\quad$ Art. 15 C.c.Q.

55. $\quad$ Blais, supra note 47 et Forcier, supra note 52.

56. Supra note 43.

57. Ibid. Pour une analyse de cette question, voir R.P. Kouri et C. Lemieux, «Les témoins de Jéhovah et le refus de certains traitements : problèmes de forme, de capacité et de constitutionnalité découlant du Code civil du Québec» (1995) 26 R.D.U.S. 77. 


\section{A. Le consentement substitué ou le consentement personnel du mineur}

\section{i) L'effet de l'âge sur le consentement}

Pour le mineur de moins de 14 ans, le consentement aux soins de santé est donné par le titulaire de l'autorité parentale ou par le tuteur ${ }^{58}$. Ce mineur est donc frappé, à cet égard, comme pour toute sa vie juridique d'ailleurs, d'une incapacité totale d'exercice ${ }^{59}$. L'article 12 C.c.Q. impose au parent ou au tuteur d'agir dans le seul intérêt du mineur ${ }^{60}$. Dans cette situation, il s'agit donc d'un régime de représentation «prévu par la loi» et mentionné à l'article 4 C.c.Q. Ainsi, l'enfant suicidaire de moins de 14 ans pourra recevoir des soins ou être hospitalisé avec le seul consentement du titulaire de l'autorité parentale ou de son tuteur.

En ce qui a trait au mineur de 14 ans et plus, le Code civil du Québec lui reconnaît la possibilité de donner un consentement valide à ses soins et traitements sans qu'il soit nécessaire d'obtenir l'autorisation du titulaire de l' autorité parentale ${ }^{61}$, ceci dans la mesure, bien entendu, où il est apte à le faire ${ }^{62}$. En cas d'inaptitude, l'autorité parentale reprend sa place. La loi reconnaît au

58. Art. 14, al.1 C.c.Q. : «Le consentement aux soins requis par l'état de santé du mineur est donné par le titulaire de l'autorité parentale ou par le tuteur».

59. Deleury et Goubau, supra note 35 à la p. 340.

60. Cette primauté est bien établie dans notre droit et est codifiée par l'article 33 C.c.Q., voir la sous-section B. au point i) «L'intervention judiciaire», ci-dessous. Voir également N. Girard, Le consentement du mineur aux soins médicaux, Cowansville (Qc), Yvon Blais, 1993 à la p. 12; A. Mayrand, «Égalité en droit familial québécois» (1985) 19 R.J.T. 249 à la p. 277; Re : Goyette : Centre de services sociaux du Montréal Métropolitain, [1983] C.S. 429 (Qc) [ci-après Re : Goyette]; Protection de la jeunesse-884, [1998] R.J.Q. 816 (C.S.).

61. Art. 14, al.2 C.c.Q. : «Le mineur de quatorze ans et plus peut, néanmoins, consentir seul à ces soins. Si son état exige qu'il demeure dans un établissement de santé ou de services sociaux pendant plus de douze heures, le titulaire de l'autorité parentale ou le tuteur doit être informé de ce fait». Voir également Lesage-Jarjoura, Lessard et Philips-Nootens, supra note 38 à la p. 176; Mayrand, supra note 43, à la p. 65; Girard, ibid. à la p. 3; R. P. Kouri, «Le consentement aux soins médicaux à la lumière du projet de loi 20» (1987) 18 R.D.U.S. 27 à la p. 36; Bernardot et Kouri, supra note 43 aux pp. 105-106.

62. Girard, supra note 60 à la p. 10; Protection de la jeunesse-884, supra note 60. 
mineur le droit de recevoir des services de santé ${ }^{63}$ et impose aux médecins et aux établissements l'obligation de lui fournir ces soins ${ }^{64}$.

L'article 14 C.c.Q. permet que les parents aient un accès limité aux informations relatives aux soins de santé de leur enfant, mais ceci ne vaut que pour l'exception qui y est mentionnée, c'est à dire qu'ils doivent être informés de l'hébergement de leur enfant dans un établissement pour une période supérieure à 12 heures ${ }^{65}$. Ils ne peuvent, selon certains, être informés des motifs à l'origine de ce maintien en établissement, à moins que le mineur n'y consente $^{66}$, car le mineur de 14 ans et plus a droit au respect du secret professionnel et son dossier est confidentiel ${ }^{67}$. Les parents peuvent en réalité avoir accès à son dossier malgré le refus de ce dernier et à certaines conditions, dans la mesure où cet accès ne serait pas de nature à causer un préjudice à la santé du mineur ${ }^{68}$.

Bien que le mineur apte de 14 ans et plus puisse consentir seul aux soins qui le concernent, il ne possède pas, à cet égard, un droit absolu ni le droit corollaire de refuser les soins en toutes circonstances. En effet, alors que le droit civil reconnaît au majeur capable et apte un droit de refus absolu de soins et de traitements $^{69}$, en dehors des cas prévus par la loi, le droit civil n'accorde au

63. Girard, ibid. aux pp. 59-60; L.S.S.S.S., supra note 27, art. 5, 7; L.P.J., supra note 10, art. 8. Ce droit a été confirmé dans l'affaire Re : Goyette, supra note 60. Voir également A. Lajoie, P.A. Molinari et J.M. Auby, Traité de droit de la santé et des services sociaux, Montréal, Presses de l'Université de Montréal, 1981 à la p. 81 et s.

64. L.S.S.S.S., supra note 27, art. 7, 13; Code de déontologie des médecins, supra note 40, art. 2.03.47; Lajoie, Molinari et Auby, ibid. à la p. 421 et s; Girard, supra note 60 aux pp. 6164.

65. Lesage-Jarjoura, Lessard et Philips-Nootens, supra note 38 à la p. 176; Mayrand, supra note 43 aux pp. 54, 62; Girard, supra note 60 aux pp. 80-85.

66. $\quad$ Girard, supra note 60 à la p. 83.

67. L.S.S.S.S., supra note 27, art. 18.

68. Ibid., art. 21.

69. Supra notes 43, 55-57. Voir également Couture-Jacquet c. Montreal Children's Hospital, [1986] R.J.Q. 1221 (C.A.) à la p. 1227 [ci-après Couture-Jacquet] (en obiter); Beausoleil, supra note 43. Malgré certaines décisions rendues en 1983 et en 1984 par la Cour supérieure dans un contexte particulier, (Institut Philippe Pinel de Montréal c. Dion, [1983] C.S. 438 [ci-après Dion]; Québec (P.G.) c. Hôpital Notre-Dame et Niemec, [1984] C.S. 426 (Qc)) la tendance récente de la jurisprudence est à l'effet que la volonté du majeur capable doit être respectée, quelles que soient les conséquences de l'absence de soins sur sa santé. 
mineur qu'un droit de refus relatif ${ }^{70}$. En effet, ce dernier ne jouit pas pleinement du droit à l'autodétermination reconnu aux adultes aptes ${ }^{71}$. Comme nous le verrons ci-dessous ${ }^{72}$, l'autorité parentale ou le tribunal, selon les circonstances, peuvent écarter son refus.

\section{ii) La suprématie de principe de l'autorité parentale}

Ainsi, les devoirs imposés aux parents par l'article 599 C.c.Q. cristallisent au plan législatif la protection du mineur. Assurer les soins et les traitements requis par l'état de santé de l'enfant fait partie de leurs devoirs ${ }^{73}$, reconnus également dans la Loi sur la protection de la jeunesse $e^{74}$ de même que dans le Code criminel $^{75}$.

L'autorité parentale est un concept d'ordre public, et les parents ne peuvent se décharger complètement de leurs responsabilités concernant les soins de santé requis par l'état de leur enfant ${ }^{76}$ :

Dans ce contexte, les parents ne sauraient, par convention, se décharger complètement de l'autorité parentale qui leur est dévolue par la loi - autorité qui leur impose des devoirs tout en leur accordant des droits - pour s'en remettre uniquement à d'autres. ${ }^{77}$

Le tribunal ne doit pas s'attribuer le droit de prendre les décisions à la place des parents ni les leur imposer et il n'interviendra que si la décision du

Voir Nancy B., supra note 43; Manoir de la Pointe Bleue (1978) inc., supra note 43. Voir également Lesage-Jarjoura, Lessard et Philips-Nootens, supra note 38 à la p. 146.

70. Lesage-Jarjoura, Lessard et Philips-Nootens, ibid. à la p. 182; Girard, supra note 60 aux pp. 180-184; Kouri et Lemieux, supra note 57 à la p. 92; Protection de la jeunesse-884, supra note 60 .

71. Girard, ibid. à la p. 180; Deleury et Goubau, supra note 35 à la p. 114.

72. Voir la sous-section B. au point i) «Par l'intervention judiciaire», ci-dessous.

73. Girard, supra note 60 aux pp. 9-10.

74. Supra note 10, art. 2.2. «La responsabilité d'assumer le soin, l'entretien et l'éducation d'un enfant incombe en premier lieu à ses parents».

75. Loi concernant le droit criminel, L.R.C. 1985, c. C-46, art. 215.

76. Re : Goyette, supra note 60 à la p. 432.

77. C. L'Heureux-Dubé, «La garde conjointe, concept acceptable ou non?» (1979) 39 R. du B. 835 à la p. 848; Re : Goyette, ibid. à la p. 432. 
titulaire de l'autorité parentale est déraisonnable ${ }^{78}$ ou constitue un manquement grave à son obligation ${ }^{79}$ :

(...) Le titulaire de l'autorité parentale est, en premier lieu et à l'exclusion de tout autre, celui qui a le pouvoir de décider au lieu et place de cet enfant. Il doit agir dans son meilleur intérêt, mais ce n'est que si, compte tenu de tous les facteurs en cause, sa décision s'avère déraisonnable et contraire aux intérêts de l'enfant qu'une Cour de justice peut intervenir. Il ne s'agit pas alors pour la Cour de se substituer au titulaire de l'autorité parentale et de décider à sa place mais de corriger une décision manifestement erronée. ${ }^{80}$

Ainsi, pour le mineur suicidaire de moins de 14 ans, le consentement aux soins de santé sera donné par le titulaire de l'autorité parentale ou par le tuteur. En pratique, ces derniers pourront consentir à des soins psychiatriques ou pharmacologiques de même qu'à une hospitalisation en cas de risque suicidaire. En cas d'empêchement ou de refus injustifié de celui qui peut consentir à des soins requis par l'état de santé du mineur de moins de 14 ans, l'autorisation du tribunal sera nécessaire pour le traiter en application de l'article 16 al. 1 C.c.Q. ${ }^{81}$

78. Art. 16, al. 1 C.c.Q.; Girard, supra note 60 à la p. 16.

79. Sur les moyens que s'est donné le législateur en cas de manquement des parents, voir la section 2 : «Les régimes d'exception», ci-dessous. Voir également Girard, ibid. à la p. 24, qui est d'opinion que le manquement par les parents en regard des soins et traitements médicaux requis par l'état de santé de leur enfant mineur donne sans doute ouverture à l'application de la Loi sur la protection de la jeunesse. À la p. 28, l'auteure indique que «confronté à un refus des parents qu'il considère déraisonnable, le médecin traitant ne doit pas hésiter et doit porter les faits à l'attention du directeur de la protection de la jeunesse». Avec respect pour l'opinion contraire, nous croyons qu'un tel signalement ne doit pas être systématique. Le médecin doit tenir compte des autres lois en vigueur et tout aussi applicables dans un tel cas, dont la Loi sur les services de santé et les services sociaux, supra note 27, particulièrement les art. 81-82 de même que l'article 16 C.c.Q.

80. Couture-Jacquet, supra note 69 à la p. 1227; Girard, supra note 60 à la p. 16.

81. Sur cette question, on peut également consulter Mayrand, supra note 43 à la p. 58 et s. Voir par exemple Protection de la jeunesse-332, [1988] R.J.Q. 1666 (C.S.). Dans cette affaire, le tribunal avait à se pencher sur une demande d'autorisation pour opérer un enfant atteint de trisomie 21 et de malformations cardiaques. Cette demande était présentée par le Directeur de la protection de la jeunesse en vertu de l'article 42 de la Loi sur la protection de la santé publique, aujourd'hui remplacée par l'article 16 C.c.Q. Les parents refusaient de consentir à l'intervention chirurgicale de leur enfant âgé de quelques jours. Cette intervention était le seul moyen qui pouvait lui permettre de survivre. Le juge, tout en 
Pour un mineur apte de 14 ans et plus, ce n'est qu'en cas d'urgence que le consentement du titulaire de l'autorité parentale ou du tuteur sera suffisant pour lui administrer des soins ${ }^{82}$ sans son consentement et même malgré son refus $^{83}$. Le concept d'urgence n'est pas défini dans la loi ${ }^{84}$. Les auteurs s'entendent cependant pour affirmer qu'il s'agit d'une situation qui ne peut être différée, où la vie ou l'intégrité corporelle est menacée et où des soins immédiats s'imposent pour les préserver ${ }^{85}$. Dans ce contexte, nous nous demandons quelle serait la limite aux soins qui pourraient être imposés par le biais d'un consentement substitué au mineur de 14 ans et plus qui présente un risque suicidaire. Dans la mesure où «la principale raison d'être de l'inviolabilité de la personne est la conservation de la vie» ${ }^{86}$ et que «[1'] atteinte à une personne pour lui éviter la mort est sans doute licite» ${ }^{87}$, il nous apparaît

reconnaissant que la vie est le plus grand bien que possède un être humain, rejetait la requête, ne pouvant se convaincre que la décision des parents était un décision injustifiée en regard de l'intérêt de l'enfant. Sur le rôle du tribunal appelé à trancher une telle question et sur la nature de la responsabilité des titulaires de l'autorité parentale, le juge s'exprime ainsi, à la p. 1669 : «La décision que les parents ont prise ne m’apparaît pas avoir été une décision capricieuse, basée uniquement sur leur situation personnelle et basée sur le fait que malheureusement leur enfant est né avec le handicap mental qu'on a décrit. À mon avis, la décision des parents, qui sont les seuls à pouvoir la prendre au point de départ, je le répète le tribunal n'intervient que si cette décision n'est pas justifiée par le meilleur intérêt de l'enfant -, cette décision fut éclairée au sens où l'entend la Cour suprême du Canada dans l'arrêt Reibl c. Hughes, et ce, [...] par un ensemble de personnes hautement qualifiées pour les éclairer.» Un peu plus tard, dans un cas de spina-bifida et d'hydrocéphalie qui, s'il n'était pas traité à brève échéance, entraînerait la mort de l'enfant né quelques jours plus tôt, le juge a également refusé d'accorder l'ordonnance requise et a rejeté la requête, indiquant que «la décision des parents a été éclairée, consciente et responsable». : Commission de protection des droits de la jeunesse du Québec c. T., [1990] R.J.Q. 1674 (C.S.). Voir également Couture-Jacquet, supra note 69, au même effet. Voir cependant l'affaire Re : Goyette, supra note 60 dans laquelle les soins de santé on été ordonnés par le tribunal malgré le refus des parents. Voir enfin Kouri et Lemieux, supra note 57 et R.P. Kouri, «Blood transfusions, Jehovah's witnesses and the rule of inviolability of the human body» (1974) 5 R.D.U.S. 156.

82. Art. 16, al. 2 C.c.Q.

83. Lesage-Jarjoura, Lesssard et Philips-Nootens, supra note 38 aux pp. 182, 184.

84. Ibid. à la p. 167.

85. Ibid. aux pp. 167-168; Bernardot et Kouri, supra note 43 aux pp. 137-138.

86. Mayrand, supra note 43 à la p. 19.

87. Ibid. 
qu'en présence d'un risque immédiat, le mineur pourrait être hospitalisé ${ }^{88}$ sans qu'il soit nécessaire d'obtenir une ordonnance. Quant à savoir si, dans le cadre de cette hospitalisation, d'autres soins, tel un traitement médicamenteux, pourraient être imposés à ce mineur pour diminuer le risque suicidaire, la réponse n'est pas claire car la jurisprudence et la doctrine sont partagées. Ainsi, le professeur Crépeau, bien qu'il ne se prononce pas spécifiquement sur cette question, accorde au droit de refus un caractère absolu ${ }^{89}$ alors que monsieur le juge Mayrand justifie l'intervention forcée dans les cas où cela est nécessaire pour protéger la personne ${ }^{90}$. La jurisprudence oscille entre le droit absolu à l'autodétermination ${ }^{91}$ et le caractère relatif qu'elle confère à ce droit, en certaines circonstances ${ }^{92}$. Pour notre part, nous croyons que l'économie générale de notre droit tend à protéger le mineur malgré lui ${ }^{93}$. Néanmoins, nous croyons que la question de l'administration des soins au-delà de ce qui est strictement nécessaire pour sauver la vie du mineur suicidaire devrait être soumise au

88. C'est d'ailleurs ce qui est prévu dans la Loi sur la protection des personnes dont l'état mental présente un danger pour elles-mêmes ou pour autrui, supra note 11, pour les 72 premières heures en cas de danger grave et immédiat. De plus, l'article 26 C.c.Q. relatif à la garde en établissement prévoit que le consentement à cette garde, pour le mineur, peut être donné par le titulaire de l'autorité parentale, sans faire de distinction quant à l'âge. Il nous apparaît donc que si le mineur présente un danger pour lui-même, l'hospitalisation pourra avoir lieu suivant le consentement du titulaire de l'autorité parentale seulement, même si le mineur de 14 ans et plus ne consent pas. Voir la section 2 : «Les régimes d'exception», cidessous.

89. P.A. Crépeau, «Le consentement du mineur en matière de soins et traitements médicaux ou chirurgicaux selon le droit civil canadien» (1974) 52 R. du B. can. 247 commenté par Lesage-Jarjoura, Lessard et Philips-Nootens, supra note 38 à la p. 145.

90. Mayrand, supra note 43 à la p. 48. Commenté par Lesage-Jarjoura, Lessard et PhilipsNootens, ibid. à la p. 145.

91. Nancy B., supra note 43 et Malette, supra note 43.

92. Blais, supra note 47; Dion, supra note 69. Ces deux décisions, de même que les affaires Nancy B., supra note 43 et Mallette, supra note 43, concernent cependant la situation de personnes majeures.

93. Par l'effet de la Loi sur la protection de la jeunesse, supra note 10, et de l'art. 16 C.c.Q. Voir également Deleury et Goubau, supra note 35 à la p. $114:$ :le mineur de quatorze ans et plus, à moins d'être inapte, peut consentir seul aux soins requis par son état de santé. Toutefois, le Code civil introduit des nuances. Sa "capacité médicale" s'apprécie en regard de la nature des soins [...] sans que jamais, pourtant, on lui reconnaisse une pleine autonomie» et à la p. $116:$ «[...] le droit du mineur de 14 ans et plus de refuser des soins requis par son état de santé s'analyse aujourd'hui comme un droit relatif.». 
tribunal $^{94}$, en application de l'article 16 C.c.Q. dont nous traiterons ci-dessous, ou de la Loi sur la protection de la jeunesse ${ }^{95}$.

\section{B. L'administration des soins de santé sans le consentement du mineur}

\section{i) L'intervention judiciaire}

Sauf urgence ${ }^{96}$, les soins requis par l'état de santé du mineur suicidaire apte de 14 ans et plus qui les refuse ne pourront lui être imposés que par le biais d'une intervention judiciaire initiée en vertu de l'article 16 al. 2 C.c.Q. :

L'autorisation du tribunal est nécessaire (...) pour soumettre un mineur âgé de quatorze ans et plus à des soins qu'il refuse, à moins qu'il n'y ait urgence et que sa vie ne soit en danger ou son intégrité menacée, auquel cas le consentement du titulaire de l'autorité parentale ou du tuteur suffit.

Ainsi, malgré le fait que le mineur reste sous l'autorité de ses père et mère jusqu'à sa majorité ou jusqu'à son émancipation ${ }^{97}$, dans l'état actuel du droit, son refus ne sera pas subordonné à cette autorité parentale mais plutôt à l'intervention du tribunal ${ }^{98}$. Appelé à statuer, ce dernier devra prendre l'avis d'experts, du titulaire de l'autorité parentale, de toute personne qui manifeste un intérêt pour le jeune concerné et devra, sauf impossibilité, recueillir l'avis de celui-ci ${ }^{99}$. De plus, cette décision devra être prise dans le seul intérêt du mineur et tenir compte, dans la mesure du possible, de sa volonté ${ }^{100}$. Avant d'ordonner que les soins soient prodigués, le tribunal devra s'assurer qu'ils seront bénéfiques et opportuns dans les circonstance et que les risques qu'ils peuvent

94. Dans le même sens, voir Lesage-Jarjoura, Lessard et Philips-Nootens, supra note 38 à la p. 184 et Girard, supra note 60 à la p. 182.

95. Voir la section 2 : «Les régimes d'exception», ci-dessous.

96. Art. 13 et 16 C.c.Q.; voir également le point ii) «Les cas d'urgence», ci-dessous et supra notes $82-85$

97. Art. 598 C.c.Q.

98. Girard, supra note 60 à la p. 182; Protection de la jeunesse-884, supra note 60 à la p. 829.

99. Art. 23 C.c.Q.; Girard, supra note 60 à la p. 185.

100. Art. 12 C.c.Q.; Protection de la jeunesse-884, supra note 60. 
présenter ne sont pas hors de proportion avec les bienfaits qu' on en espère ${ }^{101}$. Ce tribunal sera la Cour supérieure, mais nous verrons plus loin que des soins de santé pourront également être imposés au mineur par la Cour du Québec, Chambre de la jeunesse, en application de la Loi sur la protection de la jeunesse $e^{102}$.

En ce qui a trait au refus de traitement, la situation juridique du mineur apte de 14 ans et plus est donc un «état intermédiaire entre la majorité et la minorité» ${ }^{103}$. En effet, le Code civil du Québec introduit certaines nuances ${ }^{104}$. Bien qu'il puisse consentir seul aux soins requis par son état de santé, sa capacité en matière médicale sera modulée ${ }^{105}$ et la possibilité de refuser dépendra de la nature des soins proposés. S'il refuse de recevoir des soins requis par son état de santé, le tribunal pourra les lui imposer, en vertu de l'article 16 al. 2 C.c.Q.

Pour statuer, la Cour appliquera en outre l'article 33 C.c.Q. qui se lit comme suit :

\section{Art. 33 :}

Les décisions concernant l'enfant doivent être prises dans son intérêt et dans le respect de ses droits.

Sont pris en considération, outre les besoins moraux, intellectuels, affectifs et physiques de l'enfant, son âge, sa santé, son caractère, son milieu familial et les autres aspects de sa situation.

101. Ibid.

102. Supra note 10, par le biais des art. 76.1 ou 91 ; voir la section 2 :, sous-section A. «En vertu de la Loi sur la protection de la jeunesse», ci-dessous.

103. Deleury et Goubau, supra note 35 à la p. 114.

104. Ibid., Girard, supra note 60 aux pp. 185-189.

105. Ibid. À titre d'exemple, voir Protection de la jeunesse-310, [1988] R.J.Q. 1135 (Trib. jeun.), dans laquelle le tribunal, bien qu'il n'aborde pas la question sous l'angle de l'aptitude à consentir ni sous l'angle de l'article 14 C.c.Q., en arrive indirectement à cette conclusion en décidant que la décision de l'adolescente de 14 ans de mettre fin à sa grossesse était réfléchie et éclairée. Par conséquent, elle était apte. 
Dans une affaire récente ${ }^{106}$, la Cour supérieure devait trancher une requête présentée par le Directeur de la protection de la jeunesse pour autorisation de soins médicaux sur une mineure de 14 ans. Celle-ci, soutenue dans sa décision par ses parents, refusait, pour des motifs religieux, l'intervention chirurgicale qui pourrait corriger une déviation importante de la colonne vertébrale. La preuve révélait que, sans traitement, l'enfant présenterait éventuellement une insuffisance respiratoire assez sévère pour la limiter globalement, suivie d'une insuffisance cardiaque et de son décès plus que probable avant l'âge de 40 ans $^{107}$. L'intervention ne lui procurerait que $50 \%$ d'amélioration de sa condition déjà détériorée, mais permettrait d'arrêter la progression de la maladie et de mener une vie presque normale. Enfin, suivant la preuve, il était médicalement déraisonnable de refuser l'intervention dont les avantages dépassaient largement les risques prévisibles ${ }^{108}$. Le tribunal, évaluant l'aptitude de l'enfant à donner un consentement libre et éclairé, conclut à cet égard que «[sa foi en Dieu] est aveugle et nuit à [son] aptitude d'exprimer un refus libre d'une personne (mineure) capable de discernement» ${ }^{109}$. Quant à la capacité de l'enfant de refuser les soins, le tribunal considère que «la décision de M n'est pas raisonnable et qu'elle n'a pas la capacité de discerner entre l'intervention directe de Dieu sur son avenir et la réalité.(...) M est trop convaincue que Dieu la guérira pour convaincre le Tribunal [sic] que sa décision est celle d'une personne douée de discernement et de maturité suffisante pour prendre une décision aussi importante pour son avenir. Sa décision n'est pas libre» ${ }^{110}$.

Ainsi, en ce qui a trait aux mineurs de moins de 14 ans, l'État ne devrait intervenir sous la forme d'une intervention judiciaire initiée en vertu de l'article 16 al.1 C.c.Q. qu'en cas de manquement du titulaire de l'autorité parentale ${ }^{111}$. Pour ce qui est du mineur de 14 ans et plus, l'intervention judiciaire devrait être

106. Protection de la jeunesse-884, supra note 60.

107. Ibid. à la p. 819.

108. Ibid. à la p. 827.

109. Ibid. à la p. 831 .

110. Ibid. Pour une analyse de l'impact des convictions religieuses sur le respect du refus du mineur de consentir aux soins de santé, voir Kouri et Lemieux, supra note 57 et Kouri, supra note 81 .

111. Mayrand, supra note 43 à la p. 61; Deleury et Rivet, «La protection de l'enfant en droit social québécois» [1978] 9 R.D.U.S. 16, à la p. 61. 
sollicitée, par le biais du deuxième alinéa de l'article 16 C.c.Q., lorsqu'on cherche à le soumettre à des soins qu'il refuse ${ }^{112}$. Nous verrons cependant qu'en pratique, ces règles sont parfois mises de côté par l'intervention systématique du Directeur de la protection de la jeunesse ou de la Cour du Québec, Chambre de la jeunesse. En effet, l'État peut également intervenir en lieu et place des parents pour protéger un mineur qui risque de présenter un danger pour luimême et ce, en vertu de la Loi sur la protection de la jeunesse. Il faut cependant que les conditions prescrites par cette loi d'exception soient remplies, tel que traité ci-après, à la section 2 .

\section{ii) Les cas d'urgence}

Les articles 13 et 16 C.c.Q. constituent des exceptions législatives au principe d'inviolabilité de la personne. Ils permettent de prodiguer des soins médicaux sans le consentement du mineur suicidaire de 14 ans et plus et sans égard à son aptitude ${ }^{113}$, lorsque sa vie est en danger ou son intégrité menacée ${ }^{114}$.

Ainsi, c'est l'article 13 C.c.Q. qui permettra de sauver la vie d'un mineur qui aurait tenté d'y mettre fin, quel que soit son âge et dans la mesure où il n'est pas possible d'obtenir en temps utile son consentement ou celui des parents. Cependant, suivant le second alinéa de l'article 13 C.c.Q., les soins ne doivent pas être inusités ni devenus inutiles ni leurs conséquences être intolérables pour la personne $e^{115}$.

Également, suivant le second alinéa de l'article 16 C.c.Q., le consentement du titulaire de l'autorité parentale ou du tuteur sera suffisant pour traiter le mineur âgé de 14 ans et plus lorsque sa vie est en danger ou son intégrité menacée. Ainsi, dans les cas qui le permettent et suivant le degré de l'urgence, ce consentement substitué doit être obtenu.

112. Supra note 98.

113. Deleury et Goubau, supra note 35 à la p. 110.

114. Bernardot et Kouri, supra note 43 aux pp. 137-140; Lesage-Jarjoura, Lessard et PhilipsNootens, supra note 38 aux pp. 167-170; Girard, supra note 60 à la p. 59 et s.

115. Lesage-Jarjoura, Lessard et Philips-Nootens, supra note 38 à la p. 168; Deleury et Goubau, supra note 35 à la p. 110. 
Que devrait faire le médecin traitant face à un double refus, soit celui du titulaire de l'autorité parentale ou du tuteur, et celui du mineur suicidaire alors que l'urgence de la situation ne lui permet pas de saisir le tribunal? Cette situation, qui nous apparaît par ailleurs peu probable dans la problématique sous étude, pourrait se présenter dans le cas où une transfusion sanguine, par exemple, est requise sans délai pour maintenir en vie le mineur suicidaire. S'il refuse la transfusion en raison de ses convictions religieuses et est appuyé par ses parents, le médecin pourrait-il ou devrait-il administrer ce traitement malgré ce double refus? Le Code civil du Québec ne répond pas à cette question. Pour certains auteurs, le mineur de 14 ans et plus ne peut pas refuser une intervention qui serait nécessaire pour lui sauver la vie ${ }^{116}$. Les soins devraient donc être administrés ${ }^{117}$, nonobstant le refus des autres personnes habilitées à décider ${ }^{118}$, et le tribunal ultérieurement saisi pour légitimer l'intervention en cours. Pour d'autres cependant, le libellé du Code civil du Québec ne semble pas permettre de passer outre au refus du mineur de 14 ans et plus appuyé par celui des parents ${ }^{119}$. Il n'y a donc pas de réponse absolue.

\section{SECTION 2 : LES RÉGIMES D'EXCEPTION ${ }^{120}$}

\section{A. En vertu de la Loi sur la protection de la jeunesse}

L'article 2 de la Loi sur la protection de la jeunesse ${ }^{121}$ prévoit que celleci «s'applique à un enfant dont la sécurité ou le développement est ou peut être

116. Kouri, supra note 81 à la p. 168.

117. Kouri et Lemieux, supra note 57 à la p. 98; Kouri, supra note 81 à la p. 167; Mayrand, supra note 43 à la p. 61. Pour ces auteurs, le refus des parents en regard de leur enfant de moins de 14 ans ne saurait non plus empêcher l'équipe de soins de donner une transfusion sanguine.

118. Kouri et Lemieux, supra note 57 à la p. 98.

119. J.P. Ménard, «Les nouvelles règles relatives au consentement aux actes médicaux» dans Service de la formation permanente du Barreau du Québec, Congrès annuel du Barreau du Québec (1991), Montréal, Barreau du Québec, 1991, 667 à la p. 684 qui affirme que «la formulation de l'alinéa 2 de l'article 19 .4 C.c.B.C. [l'article 16 al.2 C.c.Q.] [...] ne semble pas permettre que l'on passe outre au refus des parents ou du tuteur de consentir». Voir également Protection de la jeunesse-738, [1995] R.D.F. 372 (C.Q.).

120. Voir, pour une analyse de cette question, Deleury et Rivet, supra note 111 à la p. 29 et s.

121. Supra note 10. 
considéré comme compromis». L'article 1c) définit l'enfant comme étant une personne de moins de 18 ans.

La Loi sur la protection de la jeunesse régit tous les aspects du processus portant sur la protection de l'enfant. Elle précise les responsabilités et les devoirs du Directeur de la protection de la jeunesse ${ }^{122}$, la procédure à suivre devant le tribunal ${ }^{123}$ et les exigences en matière de preuve. Quant aux objectifs de cette loi, les articles 2.3 et 3 indiquent que le but principal est d'assurer la protection de l'enfant en mettant fin aux situations qui compromettent sa sécurité ou son développement, le tout dans son intérêt et dans le respect de ses droits $^{124}$. Elle vise à assurer cette protection par des interventions de nature à promouvoir l'intérêt de l'enfant et la responsabilité des parents à cet égard, en favorisant l'implication de la communauté ${ }^{125}$. En ce sens, l'intervention de l'État doit avoir pour but de rétablir, dans les meilleurs délais, l'équilibre rompu $^{126}$.

C'est le législateur qui définit, par l'article 38 de la loi, les situations où le Directeur de la protection de la jeunesse doit intervenir, c'est-à-dire lorsque la sécurité ou le développement d'un enfant est considéré comme compromis. Cet article se lit en partie comme suit :

122. Particulièrement aux art. 31-37, 41 et 45-71, supra note 10.

123. C'est la Cour du Québec, chambre de la jeunesse, qui a juridiction dans les matières relatives à la jeunesse et ce, suivant l'article 36.1 C.p.c.

124. Sous cet aspect, il est à noter que la Loi sur la protection de la jeunesse reprend les principes énoncés aux articles 32 et 33 C.c.Q. La Cour suprême du Canada a eu l'occasion de se pencher sur l'interprétation de dispositions législatives semblables dans une décision rendue en vertu de la Loi sur les services à l'enfance et à la famille de l'Ontario, L.R.O. 1990, c. C-11 dans l'affaire C.M. c. Catholic Children's Aid Society of Metropolitan Toronto, [1994] 2 R.C.S. 165. Elle indiquait que les différentes étapes de la procédure et les garanties qui gouvernent l'ensemble du processus prévu à cette loi devaient toujours être interprétés à la lumière de son objectif, soit promouvoir l'intérêt véritable de l'enfant, sa protection et son bien-être.

125. L.P.J., supra note 10, art. 2.3-2.4.

126. Ibid., art. 2.4 . 


\section{Art. 38 :}

Aux fins de la présente loi, la sécurité ou le développement d'un enfant est considéré comme compromis :

$[\ldots]$

b) si son développement mental ou affectif est menacé par l'absence de soins appropriés ou par l'isolement dans lequel il est maintenu ou par un rejet affectif grave et continu de la part de ses parents;

c) si sa santé physique est menacée par l'absence de soins appropriés;

$[\ldots]$

e) s'il est gardé par une personne dont le comportement ou le mode de vie risque de créer pour lui un danger moral ou physique;

h) s'il manifeste des troubles de comportement sérieux et que ses parents ne prennent pas les moyens nécessaires pour mettre fin à la situation qui compromet la sécurité ou le développement de leur enfant ou n'y parviennent pas. [...] $]^{127}$

L'article 38 crée une présomption simple, donc réfragable, de compromission, c'est-à-dire que si l'enfant se trouve dans l'une ou l'autre des situations qu'il énumère, sa sécurité ou son développement est considéré comme compromis. L'enfant, les parents ou toute autre personne agissant comme titulaire de l'autorité parentale, le cas échéant, ${ }^{128}$ devra alors tenter de renverser la présomption en démontrant, par l'ensemble des circonstances, que la sécurité ou le développement n'est pas compromis.

127. Ibid.

128. Ibid., art. $1 \mathrm{e})$. 
Par ailleurs, la loi prévoit d'autres situations où la sécurité ou le développement peut être considéré comme compromis, mais la seule preuve que l'enfant est dans une ou l'autre de ces situations ne sera pas suffisante pour conclure à la compromission. Il faudra, en plus, déterminer que la sécurité ou le développement de l'enfant est compromis en raison du fait qu'il est dans cette situation. C'est ce que prévoit l'article 38.1 qui se lit en partie comme suit :

\section{Art. 38.1 :}

La sécurité ou le développement de l'enfant peut être considéré comme compromis :

a) s'il quitte sans autorisation son propre foyer, une famille d'accueil ou une installation maintenue par un établissement qui exploite un centre de réadaptation ou un centre hospitalier alors que sa situation n'est pas prise en charge par le directeur de la protection de la jeunesse;

\section{$[\ldots]$}

c) si ses parents ne s'acquittent pas des obligations de soin, d'entretien et d'éducation qu'ils ont à l'égard de l'éducation de leur enfant ou ne s'en occupent pas d'une façon stable, alors qu'il est confié à un établissement ou à une famille d'accueil depuis un an. ${ }^{129}$

Ces articles de loi, de même que tout le mécanisme de protection de l'enfant, sont mis en œuvre par le biais d'un signalement transmis au Directeur de la protection de la jeunesse par toute personne qui a un motif raisonnable de croire que la sécurité ou le développement d'un enfant est compromis. Ce signalement peut provenir de voisins, des parents ou des enfants eux-mêmes, des intervenants sociaux, scolaires ou du réseau de la santé. L'article 39 se lit comme suit : 


\section{Art. 39 :}

Tout professionnel qui, par la nature même de sa profession, prodigue des soins ou toute autre forme d'assistance à des enfants et qui, dans l'exercice de sa profession, a un motif raisonnable de croire que la sécurité ou le développement d'un enfant est ou peut être considéré comme compromis au sens de l'article 38 ou au sens de l'article 38.1, est tenu de signaler sans délai la situation au directeur; la même obligation incombe à tout employé d'un établissement, à tout enseignant ou à tout policier qui, dans l'exercice de ses fonctions, a un motif raisonnable de croire que la sécurité ou le développement d'un enfant est ou peut être considéré compromis au sens de ces dispositions.

$[\ldots]$

Toute personne autre qu'une personne visée au premier alinéa qui a un motif raisonnable de croire que la sécurité ou le développement d'un enfant est ou peut être considéré comme compromis au sens des paragraphes $a, b, c, d, e, f$, ou $h$, de l'article 38 ou au sens de l'article 38.1, peut signaler la situation au directeur.

Les premier et deuxième alinéas s'appliquent même à ceux liés par le secret professionnel, sauf à l'avocat qui, dans l'exercice de sa profession, reçoit des informations concernant une situation visée à l'article 38 ou 38.1 . $^{130}$

L'alinéa 1 de cet article crée une obligation légale ${ }^{131}$. Ceci signifie que dès que la personne a un motif raisonnable de croire que la sécurité ou le développement de l'enfant est compromis, elle doit signaler la situation. Tout doute sur l'opportunité ou non de faire un signalement doit se résoudre en faveur de l'enfant. Cependant, le premier réflexe d'un intervenant en présence d'un enfant ayant des difficultés ne doit pas être de se tourner vers le Directeur de la protection de la jeunesse mais vers le parent. Dans la mesure où le parent est

130. Ibid.

131. Commission scolaire Baldwin-Cartier c. Commission de la protection de la jeunesse (7 décembre 1990), Montréal 500-05-011419-908, J.E. 91-338 (C.S.). 
présent, concerné, prend ses responsabilités et prend les décisions qui s'imposent dans l'intérêt de son enfant, il n'y a pas lieu de signaler la situation au Directeur de la protection de la jeunesse car il n'y a pas alors de compromission au sens de l'article 38 .

\section{i) Les mesures d'urgence}

Alors qu'en vertu du droit commun, l'urgence est l'exception, sous le régime de la Loi sur la protection de la jeunesse, elle marque souvent le point de départ de l'intervention du Directeur de la protection de la jeunesse et est donc fréquemment la porte d'accès aux services sociaux. En effet, sur réception d'un signalement, le Directeur de la protection de la jeunesse doit en premier lieu déterminer si des mesures d'urgence s'imposent ${ }^{132}$. À ce titre, le directeur peut :

Art. $46:(\ldots)$

a) retirer immédiatement l'enfant du lieu où il se trouve;

b) confier l'enfant sans délai à un établissement qui exploite un centre de réadaptation ou un centre hospitalier, à une famille d'accueil, à un organisme approprié ou à toute autre personne;

Lorsque la mesure retenue est de confier l'enfant à un établissement visé au paragraphe $\mathrm{b}$ du premier alinéa, le directeur doit préciser si la mesure comporte un hébergement. L'établissement désigné est tenu de recevoir l'enfant. [nos italiques.]

Cet article permet donc une hospitalisation, sans consentement, sans ordonnance et sans examen psychiatrique ${ }^{133}$. Dans un tel cas, c'est le Directeur qui autorisera la prestation de services médicaux et des autres soins qu'il jugerait nécessaires et ce, sans le consentement des parents ni l'ordonnance du

132. L.P.J., supra note 10 , art. 45.

133. Ibid. Cet article est analogue à l'article 7 L.P.P.D., supra note 11, traité plus loin. 
tribunal ${ }^{134}$. Ceci pourra perdurer tant et aussi longtemps qu'il n'y aura pas d'opposition de la part des parents ou de l'enfant ${ }^{135}$ :

\section{Art. 47 :}

L'enfant doit être consulté sur l'application des mesures d'urgence; ses parents doivent l'être également dans toute la mesure du possible.

Si les parents ou l'enfant s'opposent à l'application des mesures d'urgence, le directeur peut les y contraindre. Il doit toutefois soumettre le cas au tribunal dans les plus brefs délais. Le directeur ne peut jamais appliquer des mesures d'urgence pendant plus de vingtquatre heures, sans obtenir une ordonnance qui en constate la nécessité. Une telle ordonnance peut être rendue par le greffier lorsque le juge est absent ou empêché d'agir et qu'un retard risquerait de causer un dommage sérieux à l'enfant. La décision du tribunal ou du greffier ne peut avoir d'effet pour une durée supérieure à cinq jours ouvrables.

Lorsque le délai de vingt-quatre heures se termine un samedi ou un jour non juridique, que le juge et le greffier sont absents ou empêchés d'agir et que leur interruption risque de causer un dommage sérieux à l'enfant, le directeur peut, sans ordonnance, prolonger l'application des mesures d'urgence qui se terminent alors le premier jour juridique qui suit. ${ }^{136}$

À cette étape, le tribunal ne doit que constater l'urgence et se prononcer sur la nécessité des mesures et n'a donc pas à déterminer si la sécurité ou le développement de l'enfant est compromis ${ }^{137}$. D'ailleurs, dans la majorité des cas, ce ne serait pas possible puisque le Directeur n'a pas encore procédé à l'évaluation de la situation, vu l'urgence, et n'est donc pas en mesure de se prononcer sur la situation des parents. Pour les mêmes raisons, le tribunal n'a

134. L.P.J., supra note 10, art. 48.

135. C. Q., Chambre de la jeunesse, Mingan, no 650-41-000015-889, 20 novembre 1989, j. Sirois.

136. L.P.J., supra note 10.

137. J.F. Boulais, Loi sur la protection de la jeunesse, Texte annoté, $3^{\mathrm{e}}$ éd., Société d'information juridique, Montréal, 1995 à la p. 219. 
donc pas à procéder à une enquête. Une preuve sur affidavit pourrait être jugée suffisante. La pratique à cet égard varie considérablement d'un district judiciaire à l'autre et suivant la disponibilité des juges. Étant cependant une mesure privative de liberté importante, le juge ou le greffier doit en constater la nécessité ${ }^{138}$.

C'est par le biais de ces deux articles et de l'article 62 que le Directeur de la protection de la jeunesse pourra imposer à un centre hospitalier de recevoir, d'héberger et de procéder à l'évaluation d'un adolescent qui présente un risque suicidaire. Il pourrait également avoir recours aux dispositions de la Loi sur la protection des personnes dont l'état mental présente un danger pour elles-mêmes ou pour autrui, traitées plus loin ${ }^{139}$. Le législateur a ainsi donné aux intervenants œuvrant en protection de la jeunesse les outils nécessaires à une intervention rapide et bien ciblée. L'intervention, à cette étape, ne devrait cependant pas aller au-delà de ce qui serait requis pour sauver la vie de l'enfant ou pour assurer la protection de son intégrité physique. En droit, les mesures à cet égard sont donc essentiellement les mêmes que celles prévues pour un adulte sous garde et il ne saurait être question de forcer l'enfant à entreprendre un traitement. Cependant, cela serait possible au stade des mesures provisoires et au stade des mesures finales ordonnées par le tribunal à l'issue de l'enquête sur la compromission, tel que nous le verrons plus loin.

\section{ii) L'évaluation de la situation}

Par la suite, le Directeur et les membres de son personnel qu'il autorise à cette fin procéderont à l'évaluation du signalement reçu en enquêtant sur la situation de l'enfant et sur ses conditions de vie ${ }^{140}$. La personne chargée de l'enquête décidera ensuite si la sécurité ou le développement de l'enfant est

138. Ibid. Contra : C. Q., Chambre de la jeunesse, Québec, no. 200-49-000091-88, 22 avril 1988.

139. Supra note 11. Voir la section 2 : au point B. «En vertu de la Loi sur la protection des personnes dont l'état mental présente un danger pour elles-mêmes ou pour autrui», cidessous.

140. L.P.J., supra note 10, art. 35.1. 
compromis $^{141}$ et elle devra prendre une décision quant à l'orientation de cet enfant vers le régime des mesures volontaires ou vers la voie judiciaire ${ }^{142}$.

La loi n'impose pas de délai au Directeur pour procéder à l'évaluation d'un signalement. Cependant, lorsque aux termes de l'enquête qu'il aura tenue en vertu de l'article 35.1, le Directeur est d'avis que la sécurité ou le développement d'un enfant est compromis, il doit saisir le tribunal dans les dix jours de sa décision si aucune entente sur des mesures volontaires n'est intervenue ${ }^{143}$.

Nous nous attarderons au régime de la judiciarisation étant donné que, sous le régime des mesures volontaires, il n'y a pas d'opposition du jeune et de ses parents et que les mesures applicables pour corriger la situation sont exactement les mêmes sous les deux régimes ${ }^{144}$. Mentionnons simplement qu'en cas de refus ou d'opposition du jeune de 14 ans et plus ou de ses parents, le Directeur doit saisir le tribunal de la situation de l'enfant ${ }^{145}$. En effet, l'enfant de 14 ans et plus doit non seulement adhérer aux mesures volontaires, mais il en est une partie signataire. Son refus entraînera le dépôt d'une procédure judiciaire et l'intervention du tribunal et ce, même si les parents acceptent de signer de telles mesures ${ }^{146}$. À cet égard, on retrouve donc les mêmes principes que sous le Code civil du Québec, à l'article 16, quant aux soins de santé qui ne pourront, sans intervention judiciaire, être imposés à l'enfant de 14 ans et plus advenant son refus. De la même façon, on ne pourra imposer à un enfant de 14 ans et plus des services psychosociaux auxquels il n'adhère pas.

141. Ibid., art. 49.

142. Ibid., art. 50.

143. Ibid., art. 52.

144. Ibid., art. 54, 91.

145. Ibid., art. 52 .

146. Ibid., art. 53.1. 


\section{iii) L'intervention judiciaire}

Entre le moment du dépôt de la déclaration de compromission et de l'audition devant le tribunal sur cette déclaration, la sécurité ou le développement de l'enfant peuvent rendre nécessaire l'application de mesures provisoires. Ce serait le cas lorsqu'un signalement a été retenu et que, en attente de l'audition, la situation s'est détériorée. Par exemple, à la suite de l'évaluation de la situation d'un adolescent, le Directeur saisit le tribunal pour le motif qu'il présente des troubles de comportement sérieux que les parents ne parviennent pas à corriger. Subséquemment, l'adolescent pose des gestes autodestructeurs ou verbalise des idées suicidaires. Dans de telles circonstances, une requête pour mesures provisoires pourra être déposée en vertu des articles 76.1 et $79^{147}$ :

\section{Art. 76.1 :}

Le tribunal peut, s'il l'estime nécessaire pour la sécurité ou le développement de l'enfant, rendre toute ordonnance pour l'exécution, pendant l'instance, de l'une ou de plusieurs des mesures applicables en vertu de l'article 91 .

Le tribunal peut, à tout moment, réviser cette décision.

\section{Art. 79 :}

En application de l'article 76.1, le tribunal ordonne l'hébergement obligatoire provisoire de l'enfant par une famille d'accueil ou un établissement qui exploite un centre de réadaptation si, après étude de la situation, il en vient à la conclusion que le maintien ou le retour de l'enfant chez ses parents ou à son lieu de résidence, risque de lui causer un tort sérieux. [...]

Une mesure d'hébergement obligatoire ne peut excéder 30 jours. Cependant, si les faits le justifient, le tribunal peut ordonner une seule prolongation pour une période d'au plus trente jours.

147. Ibid. 
Cette requête ne constitue pas un recours autonome et doit être reliée à une instance en cours, même si, dans les faits, l'audition de cette instance n'a pas débuté. Ainsi, un enfant ou un adolescent qui présente des comportements dangereux pourra faire l'objet d'une requête en vertu de ces articles. Le tribunal pourra alors ordonner toutes les mesures prévues à l'article 91 et notamment ordonner que l'enfant soit confié à un centre hospitalier pour une durée qu'il déterminera afin qu'il y reçoive les soins de santé dont il a besoin. De façon concurrente, l'enfant pourra faire l'objet d'une requête pour examen clinique psychiatrique en vertu de la Loi sur la protection des personnes dont l'état mental présente un danger pour elles-mêmes ou pour autrui, sur laquelle nous reviendrons, afin que la dangerosité soit évaluée par un médecin.

Le tribunal pourra également ordonner que l'enfant soit confié à un centre de réadaptation. Dans ce cas, l'hébergement ne pourra pas excéder 30 jours et ce délai peut être prolongé pour une période d'au plus 30 jours si les faits le justifient. Ces délais sont de rigueur et, bien que dans certains cas, il pourrait être de l'intérêt de l'enfant de les prolonger, le tribunal ne peut les excéder ${ }^{148}$. Cependant, dans les faits, les parties consentent fréquemment à leur prolongation et le tribunal prendra acte de ce consentement.

$\mathrm{Au}$ stade des mesures provisoires, le tribunal pourra ordonner exactement les mêmes mesures que celles qui seraient recherchées au terme de l'enquête sur la compromission de l'enfant, à l'exception d'une évaluation psychologique, expressément prévue à l'article 86 et qui ne peut avoir lieu qu'une fois la sécurité ou le développement de l'enfant déclaré compromis. Or, les règles de preuve ne sont pas les mêmes au stade des mesures provisoires puisque la preuve par ouï-dire est admissible ${ }^{149}$ alors qu'elle ne l'est pas lors de l'enquête au fond ${ }^{150}$. Par ailleurs, cette procédure n'a pas à être signifiée et une demande peut même être verbale, sur permission du tribunal ${ }^{151}$, ce dernier

148. $\quad$ Protection de la jeunesse-359, [1989] R.D.F. 70 (C.A.).

149. T.J., St-François, no. 450-41-000317-85, 20 juin 1985, j. Fauteux.

150. Sous réserve des règles relatives au témoignage d'un enfant prévues dans la loi. Voir L.P.J., supra note 10, art. 85.1-85.6.

151. Règles de pratique de la Cour du Québec (Chambre de la jeunesse) en matière civile et en matière d'adoption, R.R.Q. 1981, c. T-16, r. 8.1, art. 24, 26. 
pouvant fonder sa décision sur les allégations de la procédure principale ${ }^{152}$. Les parents doivent cependant être avisés sans délai qu'une mesure provisoire concernant leur enfant est prise et ce, suivant les termes de l'article 79. Cette formulation permet donc d'inférer que le législateur entrevoyait que l'audition pouvait être tenue ex parte. Dans les faits, le tribunal vérifiera si les parents ont été avisés et acceptera à cet égard la preuve d'un avis verbal.

L'enquête devant la Cour du Québec, Chambre de la jeunesse, est de nature civile et les règles applicables au fardeau de preuve sont celles relatives à la prépondérance des probabilités. Le tribunal est saisi par le dépôt d'une déclaration assermentée indiquant les faits qui peuvent justifier son intervention ${ }^{153}$ et signifiée par courrier recommandé au moins dix jours avant sa présentation $^{154}$.

L'article 77 indique que le juge doit procéder lui même à toute l'enquête qui donne ouverture à sa décision. Cette enquête doit néanmoins se dérouler en la forme judiciaire et les règles de preuve doivent recevoir application. Le juge devra donc entendre les différents témoignages et juger de leur crédibilité. Dans la majorité des cas, le Directeur sera la partie requérante et aura donc le fardeau de la preuve. Les parents et l'enfant seront les parties intimées et pourront, bien entendu, faire entendre leurs propres témoins.

\section{iv) Les mesures applicables}

Au terme de son enquête sur la compromission, le tribunal peut rejeter la déclaration de compromission ou l'accueillir et rendre l'une ou plusieurs des mesures prévues à l'article 91, lequel se lit en partie comme suit :

\section{Art. 91 :}

Si le tribunal en vient à la conclusion que la sécurité ou le développement de l'enfant est compromis, il peut, pour la période qu'il

152. C.S., Chicoutimi, no. 150-24-000003-81, 12 mars 1982, j. Trotier.

153. L.P.J., supra note 10, art. 75.

154. Ibid., art.76. 
détermine, ordonner l'exécution de l'une ou de plusieurs des mesures suivantes :

g) que l'enfant soit confié à un établissement qui exploite un centre hospitalier ou à un centre local de services communautaires ou à un organisme afin qu'il y reçoive les soins et l'aide dont il a besoin;

$[\ldots]$

i) que l'enfant reçoive certains services de santé;

j) que l'enfant soit confié à un établissement qui exploite un centre de réadaptation ou à une famille d'accueil, choisi par l'établissement qui exploite le centre de protection de l'enfance et de la jeunesse;

$[\ldots]$

Le tribunal peut, en outre :

$[\ldots]$

b) retirer aux parents l'exercice de l'autorité parentale;

$[\ldots]$

d) faire toute autre recommandation qu'il estime dans l'intérêt de l'enfant; ${ }^{155}$

$[\ldots$.

Pour un enfant dangereux pour lui-même, le tribunal pourra rendre une ordonnance de soins de santé suivant le paragraphe i) de l'article 91 accompagnée ou non d'une ordonnance d'hébergement en centre hospitalier si une preuve médicale démontre que ce sont là les mesures qui seraient de nature

155. Ibid. 
à mettre fin à la situation qui compromet sa sécurité ou son développement ${ }^{156}$. $\mathrm{Si}$, par ailleurs, la preuve est à l'effet que la problématique requiert davantage des soins de rééducation, d'encadrement ou de socialisation, alors l'ordonnance rendue en vertu du paragraphe j) de l'article 91 irait vers un hébergement en centre de réadaptation. En fait, le choix des mesures variera en fonction de la preuve, des motifs de compromission retenus par le tribunal, des besoins du jeune et de sa situation familiale. Le tribunal possède à cet égard des pouvoirs larges qui ont été déclarés constitutionnels par la Cour suprême ${ }^{157}$. Ces pouvoirs s'exerceront de façon concurrente aux pouvoirs similaires de la Cour supérieure dans la mesure où les soins de santé qui seraient ordonnés auraient un lien avec le motif de compromission ${ }^{158}$.

Sous le régime de la Loi sur la protection de la jeunesse, le juge peut ordonner que soient administrés des soins de santé à un mineur et ce, quel que soit son âge ${ }^{159}$ et à toutes les étapes du processus ${ }^{160}$. Il peut également le confier à un centre hospitalier ${ }^{161}$. En l'absence d'une ordonnance spécifique, les intervenants du centre hospitalier ne pourront lui administrer des soins de santé que si la vie ou l'intégrité de l'adolescent est menacée et que son consentement ne peut être obtenu en temps utile ${ }^{162}$. Ce serait le cas, par exemple, d'un adolescent qui a posé un geste suicidaire.

Ainsi, au stade des mesures provisoires, suivant les articles 76.1, 79 et 91, des soins de santé pourront être administrés à l'enfant sans son consentement

156. Protection de la jeunesse-599, [1993] R.J.Q. 611 (C.Q.).

157. B. (R.) c. Children's Aid Society of Metropolitan Toronto, [1995] 1 R.C.S. 315.

158. Kredl c. Québec (P.G.), [1966] R.C.S. 320. Ces pouvoirs judiciaires s'exerceront aussi de façon concurrente à ceux de la Cour du Québec, issus des art. 26-31 C.c.Q. et de la Loi sur la protection des personnes dont l'état mental présente un danger pour elles-mêmes ou pour autrui, dans les cas où le mineur présente un danger. Voir la sous-section B. «En vertu de la Loi sur la protection des personnes dont l'état mental présente un danger pour ellesmêmes ou pour autrui», ci-dessous.

159. L.P.J., ibid., art. 1, 76.1 et 91; Protection de la Jeunesse-310, supra note 105 où le Tribunal de la Jeunesse a autorisé une adolescente de 14 ans à obtenir un avortement non thérapeutique et a ordonné que ce soin lui soit prodigué.

160. L.P.J., ibid., art. $46-47$ et le point i) «Les mesures d'urgence», ci-dessus.

161. Le Directeur de la protection de la jeunesse a aussi ce pouvoir : L.P.J., supra note 10, art. 62.

162. Art. 13 C.c.Q. 
si le tribunal l'estime nécessaire pour sa sécurité ou son développement. Deux dispositions le prévoient expressément, soit l'article 91g) et l'article 91 i) qui permettraient d'imposer au mineur une cure de désintoxication, des traitements de nature psychiatrique ou psychologique et même des traitements pharmacologiques ${ }^{163}$. Ces mesures pourront également être ordonnées à titre de mesure finale pour mettre fin à la situation de compromission.

\section{B. En vertu de Loi sur la protection des personnes dont l'état mental présente un danger pour elles-mêmes ou pour autrui}

Tel qu'indiqué ${ }^{164}$, l'article 10 C.c.Q. permet de porter atteinte à l'intégrité d'une personne et de lui administrer des soins de santé sans son consentement si cette atteinte est prévue par la loi. Ainsi en est-il des articles 13, 14 et 16 C.c.Q. et des dispositions de la Loi sur la protection de la jeunesse. La Loi sur la protection des personnes dont l'état mental présente un danger pour elles-mêmes ou pour autrui et les articles 26 à 31 C.c.Q. relatifs à la garde en établissement permettent, par contre, de priver une personne de sa liberté, mais non de la traiter contre son gré. Ces dispositions s'appliquent à toute personne sans distinction fondée sur l'âge, sauf en ce qui a trait au consentement. Pour les mineurs, le consentement à la garde en établissement peut être donné par le titulaire de l'autorité parentale ${ }^{165}$. L'article 26 C.c.Q., ne fait pas la distinction prévue à l'article 14 C.c.Q. qui instaure un régime particulier pour les mineurs de 14 ans et plus. Il faut donc en conclure que le consentement à la garde en établissement du mineur qui présente un danger sera donné par le titulaire de l'autorité parentale et ce, quel que soit l'âge de ce mineur. Nous croyons qu'il n'y a pas lieu de faire de distinction là où le législateur n'en fait pas.

163. C.Q., Chambre de la jeunesse, Mingan, no. 650-41-000080-925, 8 janvier 1993, j. Tremblay.

164. Voir la section 1 : «Le régime de droit commun pour les soins de santé des mineurs», cidessus.

165. Art. 26 C.c.Q. 


\section{i) La notion de dangerosité}

La Loi sur la protection des personnes dont l'état mental présente un danger pour elles-mêmes ou pour autrui complète les dispositions du Code civil $d u$ Québec. Ces textes énoncent les critères et les causes permettant de déterminer la nécessité d'une mesure privative de liberté pour la personne dont l'état mental présente un danger. C'est par le développement de la jurisprudence sur la notion de dangerosité que nous pouvons en saisir le sens. Pourra être considérée comme dangereuse la personne qui a des intentions ou des manifestations suicidaires, ${ }^{166}$ la personne qui s'automutile, la personne qui n'a pas un bon contact avec la réalité et dont les mécanismes de défense sont fragiles ${ }^{167}$, la personne qui a peu de contrôle sur elle-même ou qui présente des épisodes d'agressivité et si les médicaments n'ont que des effets négligeables sur son état ${ }^{168}$. Cette dangerosité ne doit pas être hypothétique ou potentielle ${ }^{169}$. Il doit s'agir d'un danger grave, réel, actuel et raisonnablement prévisible ${ }^{170}$. Il s'agit d'une évaluation dans une perspective à court terme et non à long terme.

Cette loi met donc en place les mécanismes de protection pour la personne suicidaire. Pour ce faire, le médecin doit évaluer l'état mental de la personne et déterminer si elle est dangereuse pour elle même. Ce processus médical complexe repose sur un ensemble de facteurs qui interagissent et doivent être considérés dans leur ensemble ${ }^{171}$. Il n'existe pas de définition formelle et précise du risque suicidaire ni de critères absolus tant dans la loi ou dans la jurisprudence que dans la littérature médicale.

Suivant la jurisprudence, le médecin a une obligation spécifique en matière de prévention et l'appréciation du risque est la première étape dans la détermination de la prévisibilité du geste. Ainsi, le médecin pourra engager sa

166. Protection du malade mental-19, [1976] C.A.S. 356 (C. affaires sociales).

167. Protection du malade mental-7, 7 (1977) C.A.S. 235 (C. affaires sociales).

168. Protection du malade mental-1, [1989] C.A.S. 593 (C. affaires sociales).

169. Protection du malade mental-16, [1980] C.A.S. 764 (C. affaires sociales).

170. Protection du malade mental-1, [1988] C.A.S. 245 (C. affaires sociales).

171. P.A. Lafleur, «Le patient psychiatrique dangereux : définition, description, évaluation.» dans Service de la formation du Barreau du Québec dans Développement récents en droit de la santé mentale (1998), Cowansville (Qc), Yvon Blais, 1998, 21. Voir également Lalonde et al., supra note 7 aux pp. 597 et 1261. 
responsabilité professionnelle lorsque le suicide ou la tentative de suicide était prévisible et qu'il n'a pas pris les moyens pour le prévenir ${ }^{172}$. Cette appréciation doit se faire conformément aux normes reconnues par la profession ${ }^{173}$ qui exigent un examen clinique psychiatrique qui comportera une histoire clinique psychiatrique, l'examen des comportements antérieurs de la personne, le diagnostic posé, puisque ce diagnostic constitue un élément important dans l'appréciation du risque, la collaboration et la réponse du patient au traitement proposé. Une personne pourra être qualifiée de suicidaire si elle a commis une tentative de suicide ou si elle a eu des idéations sérieuses définies par des critères reliés à la fréquence, la durée, l'intensité et à l'élaboration de plans concrets $^{174}$. Le médecin devra ensuite poser un diagnostic concernant l'intensité du risque ${ }^{175}$, établir un plan de traitement et prendre les mesures appropriées pour en empêcher l'actualisation ${ }^{176}$, y compris faire les démarches requises pour obtenir une ordonnance de garde en établissement, au besoin ${ }^{177}$. De plus, le médecin devra élaborer dans son plan de traitement les mesures de surveillance appropriées à son évaluation du risque et de la prévisibilité du geste ${ }^{178}$. C'est l'établissement, par le biais de son personnel, qui devra exécuter les ordonnances médicales et aura la charge de surveiller le patient conformément

172. De Bogyay c. Royal Victoria Hospital, [1987] R.R.A. 613 (C.A.) [ci-après De Bogyay]; Cloutier c. Le Centre hospitalier de l'Université Laval, [1986] R.J.Q. 615 (C.S.) [ci-après Cloutier], conf. par [1990] R.J.Q. 717 (C.A.); Corporation de l'Hôtel-Dieu de Salaberry de Valleyfield c. Audette, [1977] C.A. 587 (Qc) [ci-après Audette]; Genest c. Théroux-Bergeron et autres, [1976] C.A. 604 (Qc) [ci-après Genest]; Painchaud c. Hôpital Charles Lemoyne, [1998] R.R.A. 426 (C.S.) [ci-après Painchaud]; J.P. Ménard et D. Martin, Le cadre juridique du traitement psychiatrique, Étude préparée pour le département d'administration de la santé de la Faculté de médecine de l'Université de Montréal dans le cadre du programme de formation continue, décembre 1980 [non publié]. Voir également Lalonde et al., ibid. à la p. 599.

173. Cloutier, ibid. et Lalonde et al., ibid. à la p. 1271.

174. Ibid.; Audette, supra note 172; Genest, supra note 172; De Bogyay, supra note 172 et Painchaud, supra note 172. Voir également Lalonde et al., ibid à la p. 599.

175. Cloutier, ibid. et Painchaud, ibid.

176. Ibid.; Audette, supra note 172; Genest, supra note 172; De Bogyay, supra note 172; Ménard et Martin, supra note 172 .

177. Cloutier, supra note 172; Richard-Thibault c. Hôpital St-Michel Archange, J.E. 81-855. (C.A.) [ci-après Richard Thibault].

178. De Bogyay, supra note 172; Cloutier, ibid; Audette, supra note 172. 
à la prescription émise et d'assurer sa sécurité ${ }^{179}$. Pour exécuter ses obligations de surveillance, l'établissement doit prendre les mesures qui s'imposent pour protéger le patient et prévenir un accident ou un suicide raisonnablement prévisible $^{180}$.

Dans la Loi sur la protection des personnes dont l'état mental présente un danger pour elles-mêmes ou pour autrui, le législateur a édicté que l'évaluation de la dangerosité doit être effectuée d'abord par un psychiatre et s'il est impossible d'obtenir les services d'un psychiatre en temps utile, l'examen peut être fait par tout autre médecin ${ }^{181}$. Le législateur ne dit pas «par toute autre personne». En droit, le Directeur de la protection de la jeunesse n'a donc pas juridiction sur cette question ni les intervenants qui ont pris en charge la situation d'un adolescent hébergé en établissement. D'ailleurs, la jurisprudence indique clairement que l'évaluation du risque suicidaire et l'adoption des mesures appropriées correspondantes sont des actes médicaux ${ }^{182}$. De plus, le législateur est présumé être un être rationnel qui reflète une pensée logique et on doit supposer que la cohérence règne entre les divers textes législatifs ${ }^{183}$. Force est de constater qu'à cet égard, la Loi sur la protection des personnes dont l'état mental présente un danger pour elles-mêmes ou pour autrui est cohérente avec les articles 81 et 82 de la Loi sur les services de santé et les services sociaux dont la lecture impose d'évidence cette conclusion ${ }^{184}$. Or, non seulement les documents de formation qui s'adressent à ces personnes ne

179. Bergeron c. Genest, [1976] C.A. 604 [ci-après Bergeron]; De Bogyay, supra note 172; Cloutier, supra note 172.

180. Bergeron, ibid; Cloutier, ibid.; Coulombe c. Hôtel-Dieu de Montréal, [1975] 2 R.C.S. 115; University Hospital Board c. Lépine, [1966] R.C.S. 561; Rizzo c. Hôpital Notre-Dame, [1975] C.S. 425 (Qc); Child c. Vancouver General Hospital, [1970] R.C.S. 477; Héritiers de la succession de Clémentine Birk c. Corporation de l'hôpital, [1977] R.C.S. 279; Richard-Thibault, supra note 177; Lortie c. Hôpital Christ-Roi, [1985] C.S. 800 (Qc); Audette, supra note 172; Centre hospitalier Robert Giffard c. Georges (28 mai 1984), Québec 200-09-000141-819, J.E. 84-511 (C.A.); Hôpital Notre-Dame c. Villemure, [1970] C.A. 538 (Qc); Villemure c. Turcot, [1973] R.C.S. 716; Langlois c. Clinique Roy-Rousseau, [1980] C.S. 586 (Qc).

181. L.P.P.D., supra note 11 , art. 2.

182. Audette, supra note 172 aux pp. 11, 18-20.

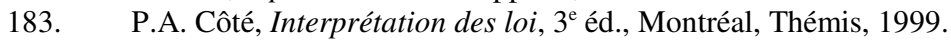

184. Voir la partie préliminaire au point ii) «Les établissements et leur mission», ci-dessus. 
tiennent pas compte de cette réalité législative ${ }^{185}$, mais ils traitent spécifiquement de l'évaluation du potentiel suicidaire ${ }^{186}$, de l'élaboration du plan de traitement et de l'établissement de mesures de protection ${ }^{187}$. Les centres Jeunesse offrent donc aux intervenants une formation qui ne respecte pas la loi, leur demandant ensuite de poser des gestes qui relèvent exclusivement de l'expertise médicale, les conduisant ainsi à excéder leurs compétences et à s'ingérer dans une mission attribuée aux établissements de santé. Ces documents devraient donc être révisés pour tenir compte de cet élément légal, incontournable pour le moment ${ }^{188}$.

\section{ii) La garde préventive et la garde provisoire}

La loi permet à tout médecin de garder en établissement, sans consentement, sans l'autorisation du tribunal et sans qu'un examen psychiatrique ait été effectué, une personne dont l'état mental présente un danger grave et immédiat pour elle-même ou pour autrui ${ }^{189}$. Cette garde préventive ne doit pas excéder 72 heures à moins que la Cour du Québec ${ }^{190}$ n'ait

185. Dans le document sur la prévention du suicide et l'intervention auprès des jeunes de l'Association des centres jeunesse du Québec, supra note 1, cet aspect est complètement ignoré. Il en va de même en ce qui a trait au document de formation que nous avons consulté soit, J. Pronovost, L. Pomerleau et D. Latreille, L'intervention auprès des adolescents suicidaires, Document de formation clinique à l'intention des intervenants en centres jeunesse, septembre 1996, [non publié] lequel a une partie intitulée «Aspects légaux de l'intervention auprès des jeunes» qui relate plusieurs dispositions législatives mais ne reflète pas du tout cette réalité.

186. Voir Pronovost, Pomerleau et Latreille, ibid. à la p. 33.

187. Ibid. à la p. 34.

188. Le Collège des médecins fait d'ailleurs une recommandation pour amender la loi afin de régler ce problème. Voir Direction générale du Collège des médecins du Québec, Accessibilité aux soins médicaux et psychiatriques pour la clientèle des adolescents : Énoncé de position, Montréal, Collège des médecins, avril 1999. Ceci ne nous apparaît pas souhaitable.

189. L.P.P.D., supra note 11, art. 7 et art. 27 al.2 C.c.Q.. Dans certaines circonstances, il ne s'agit pas seulement d'une faculté mais d'un devoir. En effet, le psychiatre a le devoir de protéger le patient suicidaire contre lui-même. S'il fait défaut d'agir alors qu'il a constaté que le patient était suicidaire, sa conduite pourrait être qualifiée de fautive et il s'expose à devoir répondre des dommages causés par son omission. Voir Cloutier, supra note 172; Ménard et Martin, supra note 172.

190. Art. 36.2 C.p.c. 
été saisie de la situation et ait ordonné une garde provisoire pour que cette personne soit soumise à une évaluation psychiatrique. Si le délai de 72 heures expire «un samedi ou un jour non juridique, qu'aucun juge compétent ne peut agir et que cesser la garde présente un danger, celle-ci peut être prolongée jusqu'à l'expiration du premier jour juridique qui suit» ${ }^{191}$.

L'article 7 de la loi permet la garde préventive sans examen psychiatrique et pourra être appliqué, dans le cas des mineurs suicidaires, par le biais de l'article 8 qui prévoit qu'un agent de la paix peut amener la personne contre son gré auprès d'un centre local de services communautaires ou d'un centre hospitalier et ce, quel que soit son âge. Cette démarche sera initiée à la demande du titulaire de l'autorité parentale ou du tuteur. De plus, la demande ne pourra être exécutée que dans la mesure où l'agent a des motifs sérieux de croire que l'état mental du mineur présente un danger grave et immédiat pour lui-même ou pour autrui.

Par la suite, le mineur pourra être gardé provisoirement pour subir une évaluation psychiatrique, si le titulaire de l'autorité parentale y consent, ou une telle évaluation pourrait être ordonnée par le tribunal, conformément aux articles 778 à 781 du Code de procédure civile ${ }^{192}$.

\section{iii) Les conditions d'obtention d'une ordonnance de garde en établissement}

Une ordonnance de garde en établissement devra toujours être précédée d'une évaluation psychiatrique ordonnée par le tribunal en application de l'article 27 C.c.Q. et de la Loi sur la protection des personnes dont l'état mental présente un danger pour elles-mêmes ou pour autrui. Cette évaluation doit être effectuée dans les 24 heures de la prise en charge de la personne concernée par l'établissement ou de l'ordonnance du tribunal si elle était déjà sous garde préventive ${ }^{193}$. Si cet examen conclut à la nécessité de garder la personne en établissement, un second examen doit être effectué par un autre médecin dans

191. L.P.P.D., supra note 11, art. 7.

192. Ibid.; art. 27-28 C.c.Q.

193. Ibid.; art. 28 al. 1 C.c.Q. 
les 96 heures de la prise en charge ou dans les 48 heures de l'ordonnance du tribunal si la personne était déjà sous garde préventive ${ }^{194}$.

La garde en établissement ne sera donc ordonnée que si le premier examen clinique psychiatrique conclut à sa nécessité et que cet examen est confirmé par un autre médecin dans le délai mentionné ci-dessus ${ }^{195}$. La garde ne sera justifiée que si la personne est dangereuse, c'est à dire que son état mental est «susceptible de mettre en danger» sa santé, sa sécurité ou celle d'autrui ${ }^{196}$.

L'importance dans notre législation du droit d'une personne à son autonomie et à sa liberté fait que les dispositions relatives à la garde en établissement seront interprétées restrictivement ${ }^{197}$ pour que ceci demeure une mesure exceptionnelle, prise en dernier recours et dans les cas où le droit de toute personne à la protection de son intégrité est menacé ${ }^{198}$. L'ordonnance doit donc être la plus courte et la moins contraignante possible et se baser uniquement sur l'état de dangerosité afin de protéger la personne elle-même ou autrui ${ }^{199}$.

194. Art. 28 al. 2 C.c.Q.; L.P.P.D., ibid. Voir également A.M. Veilleux et H. Allard, «Les recours et la représentation du patient psychiatrique selon la nouvelle Loi sur la protection des personnes dont l'état mental présente un danger pour elles-mêmes ou pour autrui» dans Service de la Formation permanente du Barreau du Québec, Développements récents en droit de la santé mentale (1998), Cowansville (Qc), Yvon Blais, 1998, 147 à la p. 154.

195. Ibid.

196. Art. 29 C.c.Q. Voir également Protection du malade mental-3, [1994] C.A.S. 657 (C. affaires sociales).

197. J.P. Ménard, «Les grands principes de la nouvelle Loi sur la protection des personnes dont l'état mental présente un danger pour elles-mêmes ou pour autrui» dans Service de la formation permanente du Barreau du Québec, Développements récents en droit de la santé mentale (1998), Cowansville (Qc), Yvon Blais, 1998, 1 à la p. 7 [ci-après Les grands principes de la nouvelle L.P.P.D.].

198. Veilleux et Allard, supra note 194 à la p. 151.

199. Ménard, supra note 197 à la p. 8. 


\section{iv) Les effets de la garde en établissement}

Une ordonnance de garde ne permet pas de traiter une personne contre son grée ${ }^{200}$, et la dangerosité n'entraîne pas nécessairement une inaptitude ${ }^{201}$. En effet, la jurisprudence a déterminé que le législateur a voulu soumettre la constatation de l'inaptitude à une évaluation particulière qui doit se faire sans égard à la dangerosité. On ne doit donc pas traiter une personne qui est sous garde en établissement sans avoir obtenu son consentement libre et éclairé, si elle est apte $\mathrm{e}^{202}$, ou un consentement substitué ou une ordonnance judiciaire, si elle est inapte ${ }^{203}$. Cependant, la dangerosité devrait être prise en considération au moment où le tribunal, après avoir déterminé que le patient est inapte, s'interroge sur l'opportunité du traitement proposé.

L'effet unique de l'ordonnance de garde, sur le plan juridique, est donc la détention de la personne sans son consentement. En principe, elle ne pourra pas quitter l'établissement. Si elle est autorisée à sortir, une ordonnance médicale devra fixer les modalités de cette sortie car le degré de surveillance requis par l'état de santé d'une personne doit faire l'objet d'une évaluation et d'une prescription médicale. En effet, la garde est une forme de soins qui comprend la surveillance et le contrôle physique de la personne ainsi qu'une observation professionnelle $\mathrm{e}^{204}$. Cette mesure ne durera cependant que pour le temps le plus court possible et nécessaire à sa protection ou à la protection d'autrui. Le tribunal fixera donc la durée de la garde mais, malgré ce délai, celle-ci prendra fin avant son expiration dès qu'elle n'est plus justifiée ${ }^{205}$. La

200. Lesage-Jarjoura, Lessard et Philips-Nootens, supra note 38 à la p. 174. Voir également Lalonde et al., supra note 7 à la p. 1263. Voir enfin les art. 10-11 C.c.Q. et supra notes 3944.

201. Ibid.; voir également Lacombe, supra note 47; Blais, supra note 47; G.(A.), supra note 48.

202. Deleury et Goubau, supra note 35 aux pp. 114-121 .

203. Voir la section 1 :, sous-section B. au point i) «L'intervention judiciaire», ci-dessus. Pour l'énoncé des critères permettant de déterminer si une personne est apte ou non à consentir à ses soins, nous référons le lecteur à la section 1 : «Le régime de droit commun pour les soins de santé des mineurs», ci-dessus, particulièrement aux notes 50-53.

204. J.P. Ménard, «L'impact de la Loi sur la protection des personnes dont l'état mental présente un danger pour elles-mêmes ou pour autrui sur le consentement aux soins» dans Service de la formation permanente du Barreau du Québec, Développements récents en droit de la santé mentale (1998), Cowansville (Qc), Yvon Blais, 1998, 217 à la p. 262.

205. Art. 30 C.c.Q. 
personne sous garde doit être soumise à des examens périodiques, établis par la loi, suivant un échéancier précis et impératif. Ainsi, la loi impose un nouvel examen 21 jours après la décision du tribunal, si la garde fixée est d'une durée supérieure, et par la suite, tous les 3 mois $^{206}$. Ces examens sont destinés à vérifier si la garde est toujours nécessaire. Cette dernière prendra fin sans autre formalité si un certificat délivré par le médecin traitant atteste qu'elle n'est plus justifiée ou en l'absence d'un examen effectué à l'intérieur du délai légal ${ }^{207}$. À part les dispositions spécifiques de la Loi sur la protection de la jeunesse déjà traitées $^{208}$, il n'existe pas d'autres moyens, dans notre législation civile, pour priver un mineur de sa liberté.

Les établissements habilités à recevoir les personnes mises sous garde sont «les établissements exploitant un centre hospitalier, un centre de réadaptation, un centre d'hébergement et de soins de longue durée ou un centre d'accueil et disposant des aménagements nécessaires pour recevoir et traiter les personnes atteintes de maladie mentale» ${ }^{209}$. Si l'établissement n'est pas en mesure de répondre à ces deux conditions, il doit diriger l'usager vers l'établissement le plus apte à lui venir en aide et donc, à le protéger. Dans notre analyse sur le mythe de la protection à la Partie II, nous verrons que les centres jeunesse du Québec ne disposent pas, à l'heure actuelle, «des aménagements nécessaires pour recevoir et traiter les personnes atteintes de maladie mentale» et suicidaires. Ils ne devraient donc pas, à notre avis, les prendre en charge.

Ceci fait état de la législation québécoise applicable en principe pour protéger le mineur suicidaire. Nous verrons, dans la section 2, que la voie de la Loi sur la protection des personnes dont l'état mental présente un danger pour elles-mêmes ou pour autrui n'est pratiquement jamais utilisée pour imposer une garde ou un examen psychiatrique à un mineur. D'ailleurs, les comités formés récemment pour se pencher sur cette problématique, ne font pas mention de

206. L.P.P.D., supra note 11 , art. 10.

207. Ibid., art. 10, 12.

208. Voir la sous-section A. «En vertu de la Loi sur la protection de la jeunesse», ci-dessus.

209. L.P.P.D., supra note 11, art. 9. 
cette loi, ou très peu ${ }^{210}$. De plus, suivant les informations obtenues auprès de la direction des communications à la Commission des affaires sociales, 5000 décisions sont rendues chaque année par la Commission. Cependant, seulement 200 d'entre elles font l'objet d'une publication annuelle et, dans ce répertoire, aucune rubrique ne concerne l'âge des personnes qui exercent un recours. Les cas des mineurs qui seraient soumis à la Commission des affaires sociales pour obtenir la révision d'une garde en établissement seraient exceptionnels, sinon inexistants $^{211}$. Lors de notre vérification dans les recueils de jurisprudence de la Commission de 1975 à 1998, nous n'avons retracé qu'une seule décision, rendue en 1985, qui concerne une mineure. Dans ses motifs, la Commission décline juridiction en faveur de la Cour du Québec, Chambre de la jeunesse ${ }^{212}$. Nous croyons qu'il s'agit là d'un malentendu de plus, concernant le traitement juridique du mineur en danger, qui pourrait faire en sorte que la protection de ce mineur et celle du majeur, dans une situation semblable, s'avère différente.

Ainsi, dans la partie II, à la section 1, nous tenterons d'analyser comment le milieu applique les lois et l'effet que pourrait avoir le respect du texte législatif, et de son esprit, sur la prévention du suicide de certains jeunes québécois particulièrement à risque. Nous verrons qu'en pratique, il existe une confusion au niveau du rôle qui doit être attribué au Directeur de la protection de la jeunesse et une forme d'étanchéité dans le réseau des services de santé et des services sociaux qui ne permet pas d'offrir au mineur la protection que la société souhaite pourtant lui donner.

210. Nous référons ici à trois comités soit : le Comité sur le suicide des jeunes formé de représentants de l'Association des centres jeunesse du Québec, du Collège des médecins du Québec et du Protecteur du citoyen, le sous-groupe de travail formé par le Collège des médecins du Québec et le comité formé par l'Association des centres jeunesse du Québec.

211. Pour qu'une demande de révision soit entendue, il faut, au préalable, qu'une garde ait été imposée. La demande de révision concerne la seconde étape d'une garde en établissement. Bien que ce constat ne permette pas de conclure que les mineurs ne font jamais l'objet d'une garde suivant cette loi, il suggère néanmoins qu'il s'agit de cas isolés et exceptionnels.

212. Protection du malade mental-1, [1985] C.A.S. 181 (C. affaires sociales). 


\section{PARTIE II : POUR UNE MEILLEURE PROTECTION : L'UTILISATION DES RESSOURCES DANS LE RESPECT DES LOIS}

Bien que ceci puisse sembler évident, mentionnons que la Loi sur la protection de la jeunesse s'inscrit dans le système législatif du Québec que nous avons expliqué dans la première partie. Ce système nous apparaît bien pensé et bien articulé par le législateur qui en a fait un tout cohérent. Pour bien utiliser les mécanismes légaux mis en place pour protéger les mineurs suicidaires, on ne peut en faire abstraction et il faut bien en comprendre la portée de sorte qu'en pratique, la mesure prise soit appropriée à la difficulté du mineur et respecte ses droits de même que les objectifs de protection visés par le législateur. Or, globalement, le cadre d'intervention actuel en jeunesse, que ce soit au niveau social ou médical, ne s'inscrit pas à l'intérieur de ce système. Le choix des mesures de protection qui seront appliquées et donc des lois qui seront utilisées dans un cas donné, semble reposer sur les connaissances des intervenants et non sur la nature de la problématique visée par le législateur. Par exemple, les intervenants des centres jeunesse appliqueront davantage la Loi sur la protection de la jeunesse qu'ils connaissent certainement mieux que la Loi sur la protection des personnes dont l'état mental présente un danger pour ellesmêmes ou pour autrui. Or, chacune des lois a sa raison d'être et vise à répondre à une situation précise. Bien que les services médicaux en santé mentale aient certainement intérêt à être mieux organisés pour répondre adéquatement aux besoins des jeunes ${ }^{213}$ qui présentent des psychopathologies et des problèmes de comportement complexes et atypiques relevant de plusieurs facteurs ${ }^{214}$, à notre avis, les difficultés de coordination actuelles reposent en grande partie sur la méconnaissance des lois dans leur ensemble, ce qui engendre une application en vase clos.

\footnotetext{
213. Voir la section 1 : «La pratique : le mythe de la protection», ci-dessous. Voir également Collège des médecins du Québec, supra note 188.

214. Ibid. à la p. 3.
} 


\section{SECTION 1 : LA PRATIQUE : LE MYTHE DE LA PROTECTION}

En novembre 1996, le Bureau du Collège des médecins du Québec mandatait un groupe de travail composé de médecins psychiatres et d'omnipraticiens pour examiner l'organisation des soins en santé mentale, l'accessibilité aux soins psychiatriques au Québec ainsi que les rôles respectifs des médecins psychiatres et des médecins de famille. Le rapport de ce groupe de travail a été adopté par le Bureau du Collège, le 15 octobre $1997^{215}$. Les problèmes particuliers relatifs aux services aux jeunes y sont mentionnés dans les termes suivants :

[...] il semble exister une difficulté à dépister les maladies mentales avant détérioration. C'est le cas de la dépression masquée sous le couvert de ce que l'on présume être une crise d'adolescence qui, trop souvent, aboutit à un suicide à la maison ou en centre de réadaptation, que le jeune soit ou non pris en charge dans le cadre de mesures de protection. Cette situation peut même s'amplifier si les réflexes professionnels sont à l'effet de se prémunir contre l'intrusion d'un autre dans son champs de compétence, ce qui entrave l'échange d'informations nécessaire pour déterminer s'il s'agit d'un problème médical ou de mésadaptation. ${ }^{216}$

Cette problématique a donné lieu à l'adoption, par le Bureau du Collège des médecins, aux trois recommandations suivantes :

* [que] les départements régionaux de psychiatrie assurent une consultation rapide aux jeunes en situation de crise, qu'ils soient dans la famille ou dans un centre d'hébergement, et établissent des liens fonctionnels avec le réseau des centres d'hébergement et de la DPJ;

* [que] le Collège amorce avec les représentants des centres jeunesse et de la DPJ des discussions visant un meilleur lien entre les interventions professionnelles à l'endroit des jeunes,

215. Collège des médecins du Québec, «Accessibilité aux soins psychiatriques et aux services en santé mentale au Québec» (1998) 37:4 Le Collège : Bulletin Officiel 23.

216. Ibid. à la p. 32. 
de façon à définir les conditions permettant de reconnaître les problèmes comportementaux suggestifs de maladie mentale et de définir les critères de référence pour diagnostic et traitement;

* [que] la problématique du suicide chez les adolescents soit étudiée de façon concertée afin de permettre aux médecins de mieux dépister les cas à risque. ${ }^{217}$

Le 12 avril 1999, le Comité sur le suicide chez les jeunes remettait son rapport $^{218}$ dans lequel il conclut

à l'existence d'un sérieux problème d'arrimage entre les divers organismes concernés ${ }^{219}$, tout particulièrement le problème de référence et d'accès aux ressources spécialisées en psychiatrie à des fins de consultation, de diagnostic, d'évaluation et de traitement de problèmes psychopathologiques, particulièrement pour les jeunes suicidaires des centres jeunesse ${ }^{220}$.

Le problème ainsi posé par les professionnels ayant à intervenir dans le milieu auprès des jeunes suicidaires amènera le Collège des médecins et l'Associations des centres jeunesse à élaborer des recommandations et des solutions que nous analyserons plus loin, sous le titre B. Auparavant, nous tenterons de faire état de la pratique au sein des établissements qui prodiguent des services de santé et des services sociaux aux mineurs suicidaires ou présentant un risque de suicide.

217. Ibid. aux pp. 32-33.

218. Association des centres jeunesse du Québec, Collège des médecins du Québec et Protecteur du citoyen, Le suicide chez les usagers des Centres jeunesse : il est urgent d'agir!, Rapport du Comité sur le suicide, Québec, avril 1999, [non publié] disponible en ligne : $<$ http//www.ombuds.gouv.qc.ca/fr/publications/rap_speciaux/suicide/index.htm > (date d'accès 26 novembre 2001).

219. Ces organismes ne sont pas précisés mais le rapport traite, à la p. 5, des «obstacles empêchant la concertation entre les deux principaux réseaux d'intervention auprès des jeunes suicidaires, soit les réseaux médico-hospitalier et psychosocial».

220. Ibid. à la p. 3. La Coroner Anne-Marie David a identifié cette difficulté et fait une recommandation spécifique à cet égard, voir supra note 19 à la p. 72. Nous y reviendrons en détail sous le point i) «La confusion des rôles», ci-dessous. 


\section{A. Une analyse critique du cadre d'intervention actuel}

Il n'est pas possible de déterminer la cause exacte du taux alarmant de suicide chez les mineurs au Québec ${ }^{221}$. Il n'existe actuellement aucune statistique officielle établissant les taux réels de décès par suicide chez les jeunes usagers du réseau des services sociaux ${ }^{222}$. Le Comité sur le suicide chez les jeunes indique dans son rapport que le problème de jonction entre le réseau des services de santé et celui des services sociaux est grave. Cependant, les informations disponibles ne permettent pas de cibler quelles actions ou omissions posées à l'égard des jeunes suicidaires seraient à l'origine des difficultés de dépistage ${ }^{223}$.

Sans prétendre être en mesure de dresser un tableau complet de la pratique actuelle des centres hospitaliers ou des centres jeunesse qui ont à intervenir auprès de mineurs qui présentent un risque suicidaire, nous pouvons néanmoins décrire certaines approches qui émanent des protocoles, des personnes et des rapports d'enquête du Coroner que nous avons consultés et qui nous permettent d'identifier deux grandes difficultés. La première est qu'en pratique, les intervenants du milieu excèdent souvent la juridiction, relativement limitée suivant la loi, du Directeur de la protection de la jeunesse. Ceci confère à ce dernier un droit de regard beaucoup trop étendu sur les enfants en besoin d'aide et occulte par la même occasion le rôle que chacun devrait jouer à l'égard de l'obtention des soins de santé du jeune. La seconde difficulté que nous identifions a également été constatée par les comités qui ont étudié la question du suicide chez les mineurs qui eux, la désigne comme étant un manque de coordination ou des problèmes d'arrimage. C'est peu dire. En fait, il n'y a actuellement aucun mode de fonctionnement qui puisse permettre au réseau

221. Suivant le rapport du Comité sur le suicide intitulé «Le suicide chez les usagers des Centres jeunesse : il est urgent d'agir!» supra note 218 à la p. 7, le Québec est l'une des provinces canadiennes où les taux de suicide chez les jeunes sont les plus élevés : au cours de l'année 1996, 112 québécois âgés de 10 à 19 ans sont décédés par suicide. De 1980 à 1996, l'augmentation du taux de suicide est passé du simple au double.

222. Ibid. à la p. 8.

223. Ibid. à la p. 6. Dans notre analyse de l'enquête publique qui s'est tenue dans le cas du décès de Y. B.-L., nous relevons plusieurs omissions, voir le point i) «La confusion des rôles», cidessous. Notons par ailleurs, tel qu'indiqué précédemment, que, pour le législateur, il n'y a pas deux réseaux distincts mais un seul. 
social et médical de communiquer, d'échanger de l'information, de se consulter ni, par conséquent, de coordonner leurs interventions.

\section{i) La confusion dans les rôles}

En ce qui a trait à la pratique en centre hospitalier, nous avons consulté plusieurs protocoles et des professionnels qui œuvrent auprès de mineurs qui refusent les traitements dans trois centres hospitaliers différents au sein du réseau de la santé, afin de cerner l'approche adoptée par eux pour parvenir, sinon à les traiter, du moins à les garder. Cette démarche nous a permis de constater que chacun s'inspire, de façon tout à fait inégale, de textes de lois qui sont mal compris. La pratique variera suivant le centre, ses priorités administratives, juridiques et thérapeutiques. Nous décrirons ci-dessous la démarche décrite par des chefs d'unités psychiatriques pour adolescents œuvrant dans des centres hospitaliers différents ${ }^{224}$.

Le premier hôpital, que nous désignerons comme étant l'hôpital A, est un centre de santé mentale affilié à une des universités de la région de Montréal. Il s'est doté d'un protocole spécifique sur cette question intitulé Politiques et Procédures relativement à la garde en établissement. Ce document est publié par la direction des services professionnels et s'adresse aux membres du C.M.D.P. ${ }^{225}$, aux chefs de départements et services et à la direction des soins infirmiers. Il a été émis le 11 avril 1986 et révisé en février 1995. Sous la rubrique «Consentement substitué limité», il est indiqué que «malgré l'autorisation du représentant du patient, dans tous les cas d'opposition de la personne concernée, qu'elle soit mineure ou majeure, l'autorisation du tribunal est requise ${ }^{226}$. Il est de plus précisé que «pour un mineur de moins de quatorze ans, on procédera par signalement à la D.P.J. Pour un mineur de 14 à 18 ans, la

224. Nous savons pertinemment que la première préoccupation de ces centres et de leurs intervenants est la sécurité et le bien-être de leurs jeunes patients. De plus, ce sont des intervenants de la santé et non des juristes. Par respect pour leur rôle et les limites inhérentes à ce rôle, nous ne nommerons pas les professionnels concernés afin que cela ne soit pas interprété comme une critique visant à les discréditer.

225. Conseil des médecins, dentistes et pharmaciens institué par l'établissement conformément à L.S.S.S.S., supra note 27, art. 213 et s.

226. Énoncé de l'art. 16 C.c.Q. 
procédure est la même que pour un adulte». Cette procédure est celle prévue à la Loi sur la protection du malade mental, aujourd'hui remplacée.

En ce qui concerne les mineurs de 14 ans et plus, la politique reprend les dispositions législatives relatives aux soins de santé. Nous avons cependant de la difficulté à saisir pourquoi un signalement devrait être effectué auprès du Directeur de la protection de la jeunesse en ce qui a trait à tous les mineurs de moins de 14 ans. Nous avons vu en effet qu'en vertu du Code civil du Québec, les parents ont le pouvoir et le devoir de consentir aux soins de santé pour leurs enfants de moins de 14 ans $^{227}$. De plus, les situations qui doivent être signalées au Directeur de la protection de la jeunesse sont régies par l'article 39 de la Loi sur la protection de la jeunesse, lequel est très large mais n'a pas du tout la portée que lui donne cette politique 228 . Ceci va bien au-delà de ce que prévoit cette loi, donne au Directeur de la protection de la jeunesse un «droit» de regard sur les problèmes de santé de tous les enfants de moins de 14 ans ayant besoin d'une garde et semble de plus imposer au Directeur de la protection de la jeunesse une responsabilité qu'il n'a pas. Cette responsabilité revient en premier lieu aux parents qui doivent prendre les décisions avec l'aide des intervenants de la santé ${ }^{229}$. Nous nous demandons quelle place on donne aux parents dans ce processus et pourquoi on leur impose l'intervention de l'État alors qu'il s'agit d'une situation hautement privée?

Par ailleurs, le Comité sur le suicide chez les jeunes indique dans son rapport :

Ainsi, lorsqu'un enfant présente des comportements suicidaires, le recours au Directeur de la protection de la jeunesse s'avère nécessaire si les parents ne prennent pas les moyens pour gérer la situation ou s'ils ne parviennent pas à prendre ces moyens, soit à cause de leurs limites personnelles, soit à cause $d u$ refus de leur enfant, âgé de 14 ans

227. Voir la partie I :, sous-section A. «Le consentement personnel du mineur», ci-dessus.

228. Voir la partie I : «La protection du mineur suicidaire : une réalité législative bien articulée», à la section 1 : «Le régime de droit commun pour les soins de santé des mineurs» à la soussection A. «En vertu de la Loi sur la protection de la jeunesse», ci-dessus.

229. Commission de protection des droits de la jeunesse du Québec c. T., supra note 81. 
et plus, de consentir aux soins et services nécessaires à sa survie même ${ }^{230}$ [nos italiques].

Suivant cet extrait, les membres de ce comité sont d'avis que l'intervention du Directeur de la protection de la jeunesse est nécessaire lorsque les parents ne parviennent pas à prendre les moyens pour corriger la situation. Ils ont raison si cette incapacité est due à leurs limites personnelles. Cependant, si elle est uniquement due au refus de leur enfant âgé de 14 ans et plus de consentir aux soins et services nécessaires à sa survie même, ils ont tort. Nous sommes tout à fait en désaccord avec de telles conclusions et sommes d'avis que cette position est contraire aux textes de lois expliqués dans la première partie de cet ouvrage ${ }^{231}$. En effet, en cas de refus, c'est l'article 16 alinéa 2 C.c.Q. qui doit s'appliquer ou la Loi sur la protection des personnes dont l'état mental présente un danger pour elles-mêmes ou pour autrui, si le mineur est dangereux. Nous verrons d'ailleurs que les membres de ce comité font, à cet égard, la même erreur que les membres du Collège des médecins et ceux de l'Association des centres jeunesse qui se sont penchés sur cette question ${ }^{232}$. Nous soumettons que de telles positions, de même que le protocole décrit ci-dessus, constituent des exemples de mauvaise utilisation des ressources sociales de plus en plus rares et coûteuses pour la collectivitée 233 et semblent être basées sur une mauvaise compréhension de lois qui nous apparaissent pourtant complémentaires. Il y aurait lieu, à cet égard, de donner effet à la volonté du législateur ${ }^{234}$.

230. Association des centres jeunesse du Québec, Collège des médecins du Québec et Protecteur du citoyen, supra note 218 à la p. 46.

231. Voir la partie I «La protection du mineur suicidaire : une réalité législative bien articulée», ci-dessus.

232. Voir la sous-section B. «Analyse critique des correctifs suggérés par le milieu», ci-dessous.

233. Dans sa troisième recommandation, le Comité sur le suicide demande «Que le ministère de la Santé et des Services sociaux alloue les ressources requises et soutienne de façon concrète l'implantation de services visant l'intervention efficace auprès des jeunes à risques suicidaires». Voir Association des centres jeunesse du Québec, Collège des médecins du Québec et Protecteur du citoyen, supra note 218 à la p. 34.

234. Supra note 231. 
En ce qui a trait à la pratique des centres jeunesse ${ }^{235}$, un de ses volets a fait l'objet d'une étude approfondie par la Coroner Anne-Marie David dans le cadre de l'enquête publique tenue sur le décès de Y. dans un centre de réadaptation $^{236}$. Cet adolescent est décédé à l'âge de 16 ans d'une asphyxie par pendaison, le $1^{\text {er }}$ décembre 1997. Nous présentons ci-dessous, notre analyse détaillée de cette enquête vu que la pratique de ce centre est semblable aux protocoles, que nous avons eu le privilège de consulter, qui émanaient des principaux établissements prodiguant des services sociaux dans la région de Montréal ${ }^{237}$.

Suivant le rapport d'enquête, la preuve a révélé que les parents de Y. avaient des antécédents psychiatriques dont des menaces suicidaires ${ }^{238}$. À l'âge de 7 ans, Y. est perçu comme un enfant exigeant, faisant des crises et des colères face à un refus ${ }^{239}$. Entre 1989 et 1992, la situation familiale est ponctuée de violence, de signalements à la Direction de la protection de la jeunesse, d'une consultation à l'hôpital en pédopsychiatrie et de déplacements entre les domiciles maternel et paternel. En septembre 1991, les intervenants sociaux

235. En 1995, l'Association des centres jeunesse recommandait spécifiquement que des protocoles de collaboration soient développés avec des partenaires susceptibles d'intervenir dans le cas d'un suicide. Voir l'étude préparée pour l'Association des centres d'accueil du Québec, Association des centres d'accueil du Québec, Samson, supra note 8 à la p. 47.

236. David, supra note 19. Cette enquête avait été ordonnée, le 21 janvier 1998, par le Coroner en chef du Québec, $\mathrm{M}^{\mathrm{e}}$ Pierre Morin, pour «clarifier les circonstances de ce décès, en informer le public et, s'il y a lieu, formuler des recommandations pour une meilleure protection de la vie humaine». Vingt témoins ont été entendus dont un seul témoin expert en pédopsychiatrie, lequel a pris connaissance des dossiers de Y. pour tracer son portrait, rechercher s'il y avait présence ou non de maladie psychiatrique et donner son opinion sur les interventions. Aucun autre témoin expert n'a été entendu, ce qui est étrange puisque les interventions qui étaient analysées avaient été effectuées par des travailleurs sociaux, des psychoéducateurs, des éducateurs et des psychologues. Aucun psychiatre ni pédopsychiatre n'est intervenu dans les mois qui ont précédé les trois gestes suicidaires posés par Y.

237. Les établissements sollicités sont : Les centres jeunesse de Montréal, de Lanaudière, des Laurentides et de la Montérégie.

238. Bien qu'il n'y ait pas de lien automatique de cause à effet entre la maladie mentale chez le parent et celle de l'enfant, les auteurs reconnaissent que c'est là un facteur de risque additionnel. Association des centres jeunesse du Québec, supra note 1 à la p. 19. Voir également Brent, supra note 7 à la p. 367. L'expert en pédopsychiatrie, dans son témoignage, constatera une accumulation «impressionnante» de facteurs de risque : David, supra note 19 à la p. 11. Voir également nos commentaires à cet égard, infra note 243.

239. David, ibid. à la p. 3. 
proposent des mesures volontaires que le père refuse de signer. Le dossier à la Direction de la protection de la jeunesse ${ }^{240}$ est alors fermé. En 1992, la situation familiale de Y. se détériore et il est placé en centre de réadaptation où il est noté que c'est un petit garçon agressif qui passe par des périodes d'automutilation ${ }^{241}$. L'évaluation psychologique révèle en outre une intelligence supérieure, un «potentiel auto et hétéroagressif» et des «indices éventuellement dépressifs» ${ }^{242}$. L'intervention en réadaptation en centre d'accueil permet une amélioration des comportements de Y. En mai 1995, la situation redevient tendue, de sorte que Y. doit de nouveau être placé en foyer d'accueil où les interventions ne permettent pas d'arrêter la détérioration de ses comportements. Il devient de plus en plus agressif, se désintéresse de son hygiène corporelle et fugue. En février 1997, il tente d'empoisonner un enfant. En mai 1997, il s'introduit par effraction chez un voisin et fracture les dents d'un pair $^{243}$. Il est alors placé en

240. Si des mesures volontaires sont proposées, ceci signifie que l'évaluation du Directeur de la protection de la jeunesse a déterminé que la sécurité ou le développement de Y. était compromis. Le refus de signer des mesures volontaires n' aurait pas dû entraîner la fermeture du dossier mais la saisine du tribunal, suivant L.P.J., supra note 10, art. 52. L'expert en pédopsychiatrie qualifiera cette fermeture de «questionnable» et la Coroner retiendra que le dossier n'aurait pas dû être fermé sans relever toutefois que cette fermeture était en marge de la loi. David, ibid. aux pp. 13-14.

241. L'automutilation est un comportement qui met en danger la santé et la sécurité de la personne, voir la partie I :, section 2 :, sous-section B. «En vertu de la Loi sur la protection des personnes dont l'état mental présente un danger pour elles-mêmes ou pour autrui», cidessus. Néanmoins, une évaluation clinique psychiatrique ne sera pas demandée. Cette omission n'est pas relevée par l'expert en pédopsychiatrie ni par la Coroner.

242. Malgré la présence d'indices d'une pathologie psychiatrique, le psychologue n'a pas demandé de consultation médicale ni d'évaluation clinique psychiatrique. Cette omission est relevée par l'expert en pédopsychiatrie qui est d'opinion, suivant la page 13 du rapport d'enquête, que les troubles de comportement sérieux présentés par Y. à ce moment «auraient dû susciter une demande d'évaluation pédopsychiatrique afin d'éliminer une pathologie psychiatrique de type épisode de dépression majeure ou syndrome de stress posttraumatique.» mais la Coroner ne fera pas de recommandation à cet égard. David, supra note 19.

243. L'agressivité sans motif connu et les fugues répétées peuvent être des motifs pour qu'une garde fermée soit ordonnée voir Protection du malade mental-19, supra note 166. Nul doute que la tentative d'empoisonner un enfant et l'atteinte à l'intégrité d'une autre personne constituent des comportements mettant la sécurité d'autrui en danger. Seule une évaluation clinique psychiatrique aurait pu permettre de déterminer si ce danger avait pour origine l'état mental de Y. et rendait nécessaire une garde en établissement. Voir la partie I :, section 2 :, sous-section B. «En vertu de la Loi sur la protection des personnes dont l'état mental présente un danger pour elles-mêmes ou pour autrui», ci-dessus. Cette évaluation n'a pas 
centre de réadaptation où ses comportements agressifs se poursuivent. Il fait trois fugues et au cours de l'une d'elles, en juin 1997, il se promène sur les rails du métro de Montréal $^{244}$. Il est alors placé dans un centre de réadaptation plus sécuritaire en raison de son agressivité verbale et physique. Suivant son témoignage, l'expert en pédopsychiatrie est d'opinion que «les décisions prises par l'intervenante sociale du moment lui apparaissent pertinentes et adaptées à l'évolution notamment la décision de placement en centre sécuritaire ${ }^{245}$. Néanmoins, selon ce témoin, un soutien multidisciplinaire comprenant un volet médical psychiatrique aurait permis un plus grand recul ${ }^{246}$. Ni l'expert en pédopsychiatrie ni la Coroner ne relèvent le fait que les comportements de Y. en février, en mai et en juin 1997, étaient susceptibles de mettre en danger sa sécurité ou celle d'autrui et que, pour ce motif, une évaluation clinique psychiatrique était indiquée afin d'éliminer ou de confirmer une maladie mentale sous-jacente et d'instaurer, le cas échéant, le traitement requis. Aucune

eu lieu et cette omission n'est pas relevée par la Coroner ni par l'expert en pédopsychiatrie, voir nos commentaires à cet égard, infra note 245.

244. David, supra note 19 à la p. 8. L'expert en pédopsychiatrie précise, à la p. 12, que «ce comportement peut être interprété comme un trouble des conduites ou un comportement suicidaire, ou une association des deux. Fin juin 1997, le diagnostic de troubles des conduites peut être fait en considérant l'évolution de la dernière année».

245. Ibid. à la p. 14. Nous ne pouvons comprendre cette opinion qui, par ailleurs, nous apparaît paradoxale. Dans la mesure ou l'expert en pédopsychiatrie est d'avis que le fait de se promener sur les rails du métro est un «comportement [qui] peut être interprété comme un trouble des conduites ou un comportement suicidaire, ou une association des deux» et que, par conséquent, «[fin] juin 1997, le diagnostic de troubles des conduites peut être fait en considérant l'évolution de la dernière année», il nous apparaît que la conduite à suivre à ce moment était de faire «une demande d'évaluation pédopsychiatrique afin d'éliminer une pathologie psychiatrique».

246. Ibid. 
recommandation en ce sens n'est formulée ${ }^{247}$ pour que, dans le futur, une semblable omission soit corrigée ${ }^{248}$.

Le 30 juin 1997, Y. intègre temporairement le centre où il finira par se suicider. Au début, sa situation s'améliore graduellement, suivant la preuve ${ }^{249}$, et il ne montre aucun indice de dépression ${ }^{250}$. À compter du mois de septembre, son éducateur-parrain ${ }^{251}$ note qu'il est «dépressif $+++{ }^{252}$ et ses comportements amènent des retraits en chambre. Alerté par certains propos de Y., l'éducateur jette un coup d'œil attentif dans sa chambre et trouve un lacet placé autour de la poignée de porte. Le jeune nie alors songer à se suicider ${ }^{253}$. Néanmoins, sachant qu'il s'était déjà promené sur les rails du métro, son éducateur s'inquiète et instaure une surveillance spéciale de nuit (aux trente minutes, de façon irrégulière) et déplace Y. dans une chambre contiguë au bureau des éducateurs. Par la suite,Y. commence à se dévaloriser, à se renfermer sur lui-même et à

247. Parce qu'elle est d'avis qu'il eut été préférable que Y. soit réévalué en pédopsychiatrie en 1991 et 1992, et qu'un psychiatre soit impliqué dans l'analyse du cas, la Coroner recommande simplement que «2) Le Ministère de la Santé et des Services sociaux [...] s'assure que, lors de la prise en charge d'un jeune par une DPJ, les travailleurs sociaux puissent recourir à une consultation auprès d'un psychiatre afin d'évaluer l'ensemble de la situation et les orientations à prendre», passant sous silence le fait que le risque suicidaire ou le risque homicidaire que suggèrent les comportements de Y. dans la période visée par cette recommandation n'a jamais été évalué.

248. De plus, bien que l'expert en pédopsychiatrie constate dans l'histoire de Y. une accumulation «impressionnante» de facteurs de risque propices à l'éclosion de troubles psychiatriques dont une psychopathologie parentale avec des antécédents suicidaires et les risques génétiques et relationnels qui en découlent, une discorde parentale, une incohérence éducative, des abus physiques et une vulnérabilité de l'enfant propre à son tempérament et alors qu'une hypothèse d'un épisode dépressif peut être évoquée en 1996, les décisions prises par l'intervenante lui sont apparues «pertinentes et adaptées à l'évolution» sans qu'une consultation médicale n'ait été sollicitée : David, supra note 19 à la p. 11.

249. Le 31 août 1997, Y. frappe un pair à coups de poings. Voir nos commentaires supra note 243.

250. David, supra note 19 à la p. 17. L'expert précise cependant que Y. souffrait de troubles des conduites. Or, «on insiste beaucoup sur la comorbidité [association] troubles des conduites/dépression majeure et sur l'impact majeur que cette comorbidité a sur le traitement et le pronostic de la dépression majeure» (à la p. 21).

251. Celui qui a la responsabilité quotidienne du jeune, ibid. à la p. 15.

252. Ibid. à la p. 19. L'expert en pédopsychiatrie indique, à la p. 21 du rapport d'enquête, que cette période est dépressive, triste mais non pathologique.

253. Ibid. à la p. 20. Cet incident est interprété comme étant le premier geste suicidaire posé par Y. Il en commettra trois sur une période de deux mois et demi. 
présenter une confusion de sentiments et, suivant l'expert en pédopsychiatrie, des indices de dépression majeure qui auraient mérité une consultation auprès d'un psychiatre le plus tôt possible ${ }^{254}$.

En octobre, l'agressivité de Y. monte et on lui passe les menottes pour le conduire en salle d'isolement ${ }^{255}$. En novembre, il est déprimé, souffrant, se dénigre et est plus agressif. Le 28 novembre, vers $22 \mathrm{~h} 20$, l'éducateur, ayant entendu un bruit, se rend dans la chambre de l'adolescent et voit Y. dissimulant un cordon de pantalon. Ce dernier indique avoir tenté de se pendre au protecteur du détecteur de fumée de sa chambre. L'éducateur demande alors à la personne responsable ${ }^{256}$ que l'adolescent soit vu en urgence en psychiatrie ${ }^{257}$ ou qu'il ait une surveillance constante. L'intervention de cette personne dure environ 15 minutes et Y. refuse ces deux propositions ${ }^{258}$, mais s'engage à ne rien faire cette nuit-là. Son éducateur lui promet donc que dès le lundi il sera vu par «un psychologue... une personne qui pourrait lui donner de l'aide» ${ }^{259}$ et il fait avec

254. Ibid. à la p. 22. La Coroner conclut que les démarches auraient du êtres faites pour que Y. soit vu le plus tôt possible en psychiatrie mais aucune recommandation sur cette question n'est formulée. Elle ne relève pas que la législation imposait une telle consultation, en vertu de la L.S.S.S.S., supra note 27, art. 5, 7; L.P.J., supra note 10, art. 8; Charte des droits et libertés de la personne, supra note 41, art. 1-2; L.P.P.D., supra note 11, art. 7. Voir à cet égard à la partie I :, section 2 :, sous-section B. au point i) «La notion de dangerosité», cidessus.

255. L'expert en pédopsychiatrie et la Coroner retiennent, pour cette période, une amélioration significative des comportements et une absence de signes dépressifs. David, supra note 19, à la p. 24.

256. L'intervenant a donc appliqué la Procédure relative aux manifestations de tendances suicidaires observées chez un usager du Centre [de réadaptation], émise le $1^{\text {er }}$ février 1994 qui prévoit que l'éducateur transmette les informations à la personne en responsabilité. Ce protocole est semblable au modèle de politique-cadre et de procédures proposé dans l'annexe IV de Association des centres d'accueil du Québec, supra note 8.

257. David, supra note 19 à la p. 28. À notre avis, c'est ce qu'imposait la loi. Voir nos commentaires à cet égard, partie I :, section 2 :, sous-section B. «En vertu de la Loi sur la protection des personnes dont l'état mental présente un danger pour elles-mêmes ou pour autrui», ci-dessus et supra note 254.

258. Il aurait été possible de passer outre à son refus suivant L.P.P.D., supra note 11, art. 7 .

259. Tel qu'indiqué, il nous apparaît que l'évaluation d'un risque suicidaire est du champ de pratique exclusif du médecin, suivant L.P.P.D., ibid., art. 2. Voir nos commentaires à cet égard, partie I :, section 2 :, sous-section B. «En vertu de la Loi sur la protection des personnes dont l'état mental présente un danger pour elles-mêmes ou pour autrui», cidessus. 
lui un pacte de non suicide jusqu' au lundi matin et fouille sa chambre ${ }^{260}$. On instaure alors une surveillance irrégulière aux 10 ou 15 minutes et les éducateurs doivent s'assurer «que les espadrilles de Y. demeureraient à l'extérieur de sa chambre et que sa chambre serait fouillée pour vérifier qu'il ne disposait d'aucun moyen pour attenter à sa vie ou se mutiler» ${ }^{261}$.

Nous ignorons si la personne responsable a procédé à l'évaluation du risque suicidaire. Son rôle est d'analyser «la situation et [de mettre] en place des moyens permettant la sécurité du jeune» ${ }^{262}$ ainsi que de rédiger un rapport sur l'événement. La procédure ne précise pas comment la situation doit être analysée ni quels moyens doivent être mis en place, pas plus qu'elle ne mentionne la nécessité d'une consultation médicale. Dans le modèle de politique-cadre et de procédures proposé dans l'étude «Le phénomène du suicide chez les adolescents en centre de réadaptation» ${ }^{263}$, les mêmes silences et les mêmes omissions peuvent être constatés. Il nous apparaît qu'une évaluation clinique psychiatrique était alors requise pour déterminer si l'état mental de Y. pouvait présenter un danger ${ }^{264}$.

Pour l'expert en pédopsychiatrie, Y. connaît à ce moment un épisode de dépression majeure, d'installation très rapide, propre aux adolescents ${ }^{265}$. Elle est

260. David, supra note 19 à la p. 29.

261. Ibid. Suivant la Procédure relative aux manifestations de tendances suicidaires observées chez un usager du Centre [de réadaptation], supra note 256. Il devait cependant aviser les parents, ce qui n'a pas été fait.

262. Suivant la Procédure relative aux manifestations de tendances suicidaires observées chez. un usager du Centre [de réadaptation], ibid.

263. Samson, supra note 8.

264. Voir la partie I :, section 2 :, sous-section B. «En vertu de la Loi sur la protection des personnes dont l'état mental présente un danger pour elles-mêmes ou pour autrui», cidessus.

265. Elle précise que le diagnostic de dépression majeure chez l'adolescent est très difficile à établir car les symptômes peuvent s'organiser très rapidement. Nous rappelons que jusqu'à $15 \%$ des sujets atteints de Trouble dépressif majeur meurent de suicide, supra note 25 . C'est pour cette raison, qu'à notre avis, l'évaluation d'un risque suicidaire devrait rester dans le champ de pratique exclusif des médecins. Voir la partie I :, section 2 :, sous-section B., i) «La notion de dangerosité», ci-dessus. 
d'avis que la tentative de suicide était «grave, sérieuse» ${ }^{266}$. Elle croit aussi que la décision de le garder ce soir-là dans l'unité était «recevable», [ce avec quoi la Coroner est d'accord] indiquant que «l'éducateur avait une perception intuitive extrêmement juste de ce qui se passait pour Y.» ${ }^{267}$. Nous rappelons que l'évaluation d'un risque suicidaire est un processus médical complexe qui doit conduire à un diagnostic concernant l'intensité du risque suicidaire et à la prescription de mesures pour empêcher l'actualisation du risque ${ }^{268}$. La perception intuitive de l'éducateur ne peut pas suppléer à l'examen médical, requis dans les circonstances, et le pacte de non suicide ne peut pas remplacer une prescription médicale adéquate. En plus, la Coroner indique qu'une surveillance constante n'était pas possible dans ce centre de réadaptation ${ }^{269}$.

Étant donné que la responsable du service de permanence décide qu'il n'est pas urgent que Y. soit vu en psychiatrie durant la fin de semaine, il n'est pas conduit à l'Institut Albert-Prévost ${ }^{270}$. Le lundi suivant, le chef d'unité

266. David, supra note 19 à la p. 32. Nous rappelons que la Loi sur la protection des personnes dont l'état mental présente un danger pour elles-mêmes ou pour autrui, supra note 11, permet une garde préventive, sans ordonnance ni consentement lorsque le danger est grave et immédiat.

267. Avec respect pour son opinion, nous croyons que l'expert en pédopsychiatrie fait là une erreur. Y. a présenté un comportement de nature à mettre sa vie en danger et la conduite à suivre était d'obtenir un examen clinique psychiatrique, ce qui n'a pas été fait et ne pouvait être fait dans l'unité ce soir-là. Elle omet ainsi de se prononcer sur la question essentielle, à notre avis, soit qu'il devait être vu en psychiatrie et hospitalisé.

268. Voir supra notes 171, 175-178.

269. David, supra note 19 à la p. 53.

270. Le Centre Jeunesse a un service de consultation composé de psychologues, de criminologues et d'un psycho-éducateur. Il n'y a pas de médecin, ni de psychiatre attaché à ce service, mais un psychologue dont la tâche consiste à offrir des consultations psychologiques de jour du lundi au vendredi. La décision d'une consultation en psychiatrie relève du chef d'unité qui demandera qu'un psychologue voit le jeune pour être conseillé sur la nécessité et l'urgence d'une consultation en psychiatrie. Suivant l'évaluation qui est faite de l'urgence de la situation, le jeune est vu en urgence le jour même, dans les jours suivants ou pas du tout. En cas d'urgence, le jeune peut être amené à l'Institut Albert-Prévost. La fin de semaine et les soirs, cette décision relève du responsable de Service de la permanence, ibid. aux pp. 3334 , une personne dont on ignore la formation et les compétences mais qui n'est pas médecin et qui, dans le cas qui nous occupe, a vu Y. 15 minutes le soir où il a fait cette seconde tentative de suicide. 
demande une consultation d'urgence en psychologie ${ }^{271}$ pour évaluer le risque suicidaire. La psychologue désignée ${ }^{272}$ ne prend pas connaissance du dossier de Y. avant de commencer son évaluation ${ }^{273}$. Lors de la rencontre, Y. admet sa tentative de suicide, accepte de suivre une psychothérapie et s'engage à avertir les psychoéducateurs si cela ne va pas bien du tout, ce qui, pour la psychologue, est un pacte de non suicide ${ }^{274}$. Suivant son témoignage, la psychologue interprète la tentative non pas comme une volonté réelle de mourir, mais plutôt comme un appel à l'aide ${ }^{275}$. Elle ignore alors s'il a déjà commis des gestes suicidaires dans le passé mais retient que le geste n'a pas été létal car le lien de pendaison s'était cassée ${ }^{276}$. Elle pense à la psychiatrie mais elle ne voit pas cela comme une urgence immédiate. Le plus urgent est la surveillance et elle insiste pour une surveillance plus serrée ${ }^{277}$. L'urgence en psychiatrie est «une

271. Conformément à la Procédure relative aux manifestations de tendances suicidaires observées chez un usager du Centre [de réadaptation], supra note 256.

272. Nous précisons que tous les professionnels impliqués dans ces démarches le lundi matin étaient à ce moment convaincus que Y. avait tenté de se suicider le vendredi soir, mais avait échoué en raison du fait que le lien qu'il avait utilisé s'était rompu.

273. La Coroner indique que la psychologue aurait dû au moins discuter avec l'éducateur, David, supra note 19 à la p. 42. Tel qu'indiqué dans la Section 2, l'examen pour apprécier le risque suicidaire comporte, entre autres, une histoire clinique psychiatrique et l'examen des comportements antérieurs de la personne, supra note 174. Voir également Lalonde et al., supra note 7 à la p. 599.

274. Elle aurait dû s'en assurer auprès du jeune, suivant la Coroner : David, ibid. à la p. 43.

275. Pour elle, lorsqu'une personne veut réellement mettre fin à ses jours, elle va s'assurer «qu'il n'y a pas 15 personnes autour» et dans une situation «où des gens peuvent intervenir, on est porté à évaluer ça un peu moins grave que si quelqu'un a loué une chambre d'hôtel et il s'est enfermé là». Ibid. à la p. 37. Or, l'étude effectuée par l'Association des centre d'accueil du Québec révèle que 56\% des tentatives de suicide se sont produites dans la chambre des jeunes, à l'intérieur du centre de réadaptation, $67 \%$ le soir et $66 \%$ du lundi au vendredi, voir Association des centres d'accueil du Québec, supra note 8 à la p. 17. Ce document n'a pas été déposé en preuve. Cependant, l'expert en pédopsychiatrie indique, à la p. 44, que cette tentative avait beaucoup d'importance car elle avait été secrète. Ce témoin ne tient pas compte de toutes les tentatives de suicide en établissement ni de la réalité de Y. qui, de toute façon, ne pouvait pas sortir. Enfin, elle semble confondre volonté suicidaire et difficulté de commettre le geste en institution. Elle a tort, comme l'histoire va le démontrer, puisqu'il va réussir son troisième geste.

276. La Coroner relève ce paradoxe de la psychologue qui croyait que Y. ne voulait pas réellement mourir mais si le lien ne s'était pas cassé, il serait mort. Ibid. à la p. 41.

277. Ibid. à la p. 38. Elle ignore si une garde à vue aurait été possible à ce centre de réadaptation et ne l'a pas demandé. De fait, il n'y a pas de procédure écrite quant à ce qui doit être fait, lors d'une surveillance «particulière» (à la p. 49) et la garde à vue n'est jamais utilisée sauf 
expérience traumatisante pour l'adolescent à éviter le plus possible à moins d'être totalement convaincue ${ }^{278}$. Pour le témoin psychologue, le but de la consultation en psychiatrie est de déterminer l'opportunité d'une médication ${ }^{279}$. Y. est suicidaire, à son avis, mais ambivalent ${ }^{280}$. Pour l'expert en pédopsychiatrie, la condition de Y. nécessite une consultation d'urgence en psychiatrie $^{281}$.

Par la suite, le chef d'unité fait une demande d'évaluation en pédopsychiatrie qui doit avoir lieu le lendemain ou le surlendemain ${ }^{282}$. On instaure donc une surveillance «particulière» ${ }^{283}$. Étant donné que Y. n'a pas

en présence d'une situation de crise (à la p. 43).

278. Ibid. Le témoin ne précise pas de quoi elle devait être convaincue. Elle voulait prendre le temps de préparer Y. à une consultation en psychiatrie pour que soit évaluée la pertinence de donner une médication, des antidépresseurs, sinon il aurait pu refuser de parler au psychiatre. Or, les psychiatres sont formés pour pallier à cette difficulté. Elle n'a pas demandé à Y. s'il acceptait de voir un psychiatre.

279. Ibid. à la p. 39. Elle ne sait pas que le psychiatre doit également évaluer le risque suicidaire, poser un diagnostic et instaurer un traitement qui peut consister en de la médication mais également en la prescription de mesures de surveillance proportionnelles au risque que présente le patient, supra notes 175-177. Ce témoin ignorait aussi l'existence de la Loi sur la protection du malade mental, supra note 11 . Voir également nos commentaires sous le texte correspondant aux notes 181-182. Pour sa part, l'expert en pédopsychiatrie dans son témoignage, à la p. 44, indique que l'hospitalisation suite à une tentative de suicide se justifie pour valider le diagnostic de dépression majeure et pour la mise en route du traitement, passant sous silence la protection de la vie du patient dangereux. La Coroner précise, à la p. 43, que la consultation en psychiatrie aurait pu permettre d'éliminer une maladie psychiatrique qui serait à l'origine des idéations suicidaires.

280. David, ibid. à la p. 39.

281. Ibid. à la p. 44. La Coroner, à la p. 45, indiquera qu'au mieux, Y. aurait dû être amené à l'urgence immédiatement après la consultation avec la psychologue ou au pis, il aurait dû être gardé à vue jusqu'à cette consultation le mardi. Elle ne relève pas que l'omission constituait une violation de plusieurs lois, supra note 254. Nous croyons que Y. aurait dû être conduit à l'urgence dès que les éducateurs ont perçu les gestes comme sérieux, soit le soir où le lacet a été retrouvé sur la poignée de porte et à nouveau le vendredi soir lors de sa seconde tentative de suicide.

282. Ibid. à la p. 40.

283. Notons que plusieurs hôpitaux possèdent des protocoles de surveillance qui s'appliquent suivant une ordonnance médicale et qui prévoient le degré de surveillance requis pouvant aller à toutes les demi-heures jusqu'à une surveillance constante et dans ce cas, le patient n'est jamais laissé seul. Dans ces protocoles, le type de surveillance est non seulement qualifié, mais il est également défini. 
besoin de médication ${ }^{284}$, la psychiatrie devient moins urgente. Enfin, Y. n'est pas inclus dans les cas d'enfants ayant des tendances suicidaires car son agressivité a toujours été dirigée contre les autres et en aucun temps contre lui ${ }^{285}$.

Malgré la consigne à l'effet que les espadrilles de Y. doivent lui être retirées pour assurer sa sécurité en raison de sa première tentative suicidaire avec les lacets, malgré le fait que les éducateurs savent qu'il doit faire l'objet d'une surveillance «particulière» ${ }^{286}$, Y. est laissé seul aux toilettes d'abord, puis ensuite, dans sa chambre, toutes lumières fermées, ce qui facilite le passage à l'acte : il se pend avec les lacets de ses espadrilles entre $19 \mathrm{~h} 45$ et $19 \mathrm{~h} 59^{287}$.

Ainsi, alors que le mandat spécifique du centre de réadaptation était d'assurer la protection de Y., un mineur suicidaire, de lui prodiguer des services de nature psychosociale et de s'assurer que les services requis par sa condition soient évalués et lui soient offerts ${ }^{288}$, alors qu'il avait besoin de soins de santé et devait voir un médecin, Y. est décédé sans qu'on ait ni évalué ce besoin ni tenté d'y répondre. Dans la pratique de ce centre de réadaptation et suivant son protocole, il est demandé aux éducateurs d'intervenir auprès de jeunes suicidaires, d'évaluer le risque et de prendre des mesures, sans avoir éliminé au préalable une maladie sous-jacente. On leur demande de distinguer une problématique de nature psychosociale d'une problématique médicale et de décider si le jeune doit être vu par un médecin ${ }^{289}$, jouant ainsi eux-mêmes le rôle du médecin, alors qu'ils n'ont aucune formation médicale ${ }^{290}$, et privant, par la même occasion, le jeune des services de santé auxquels il a droit.

Enfin, nous devons souligner qu'à la suite du dépôt du rapport du Coroner, le père a intenté une poursuite en responsabilité civile contre les

284. Ses propos à cet égard rejoignent ceux de la psychologue, supra note 279.

285. David, supra note 19 à la p. 41. Ce témoin ignore aussi que les comportements dangereux, que ce soit envers soi-même ou envers les autres, donnent ouverture à l'application de la L.P.P.D., supra note 11.

286. Ce que chacun interprète à sa façon mais une surveillance constante est exclue : David, ibid. à la p. 53.

287. Ibid. à la p. 55.

288. L.S.S.S.S., supra note 27 , art. 82.

289. David, supra note 19 à la p. 67.

290. Ibid. à la p. 66. 
centres jeunesse, leur reprochant un décès qui n'aurait pas dû se produire ${ }^{291}$. Sans nous prononcer sur l'issue de ce litige, nous rappelons que parfois, le suicide est un événement qui n'est pas prévisible. Dans cette éventualité, nul ne saurait en être tenu responsable. Cependant, si le suicide est prévisible, la responsabilité de ceux qui avaient le devoir de le prévenir est engagée s'ils n'ont pas pris les moyens pour éviter le passage à l' acte ${ }^{292}$. Il ne fait aucun doute dans notre esprit que c'est le cas lorsque la loi est violée. Les praticiens qui œuvrent auprès des mineurs particulièrement à risque sont dans une situation précaire face à cette réalité. Elle devrait, à notre avis et pour le bien de tous, changer le plus tôt possible.

\section{ii) La pratique en vase clos}

Le troisième exemple dont nous souhaitons traiter concerne la pratique d'un hôpital pour enfant de la région de Montréal, que nous désignerons comme étant l'Hôpital B, où il n'existe pas de politique ni procédure écrite. En pratique, pour les enfants suicidaires de moins de 14 ans, on fera appel aux parents pour obtenir les consentements nécessaires au traitement ou à la garde selon les besoins ${ }^{293}$. En cas de refus des parents, ce refus sera respecté, à moins d'une raison grave justifiant l'intervention du Directeur de la protection de la jeunesse et, dans ce cas, la situation serait signalée. Pour les enfants de 14 ans et plus, qui refusent les traitements proposés, le pédopsychiatre pourra procéder par signalement à la Direction de la protection de la jeunesse dans les cas où le jugement du patient est altéré ou s'il présente un danger pour lui-même ou pour autrui.

Cette pratique témoigne de la mauvaise compréhension des lois. Si le jugement du patient est altéré, le consentement aux soins est donné par le titulaire de l'autorité parentale ${ }^{294}$. Si le patient refuse et que son jugement n'est pas altéré, l'autorisation du tribunal est nécessaire ${ }^{295}$. S'il présente un danger et

291. Dossier numéro 540-05-004507-996.

292. Lalonde et al., supra note 7 à la p. 599.

293. Conformément à l'article 14 C.c.Q.

294. Suivant l'article 14 C.c.Q.; supra notes 58, 62.

295. Suivant l'article 16 C.c.Q., voir la partie I :, section 1 :, sous-section B. au point i) «L'intervention judiciaire», ci-dessus. 
qu'il y a urgence, l'article 16 C.c.Q. s'applique et le consentement du titulaire de l'autorité parentale ou du tuteur suffit sinon, ce sera la Loi sur la protection des personnes dont l'état mental présente un danger pour elles-mêmes ou pour autrui qui s'appliquera. A priori, il n'y a donc pas lieu de faire appel au Directeur de la protection de la jeunesse.

Dans l'Hôpital B, le signalement se fera, dans la plupart des cas, avec les parents qui collaboreront à ce type d'intervention. La suite des événements dépendra de la position qu'adoptera le Directeur de la protection de la jeunesse. On indique cependant que cette voie n'est pas courante en raison du fait que les parents ont un pouvoir de persuasion efficace envers leur enfant et que, de façon générale, ce dernier finit par consentir ${ }^{296}$.

Dans cet hôpital, la Loi sur la protection des personnes dont l'état mental présente un danger pour elles-mêmes ou pour autrui n'est jamais utilisée pour les mineurs suicidaires car on considère que le processus est plus lourd et plus long pour les obliger à se faire traiter. En effet, cette loi ne permet d'obtenir que la garde en établissement ${ }^{297}$ et non une ordonnance de traitement, imposant ainsi une deuxième démarche judiciaire. L'avantage pour l'Hôpital B d'un signalement au Directeur de la protection de la jeunesse est la saisie de la Cour du Québec, Chambre de la jeunesse. Les intervenants de la santé qui reçoivent un mineur sous ordonnance de celle-ci considèrent, à tort à notre avis, que l'ordonnance de traitement est implicite à l'ordonnance de placement en centre hospitalier. Ils sont par conséquent tout à fait à l'aise de traiter le mineur et ce, même si le jugement ne l'ordonne pas spécifiquement ${ }^{298}$.

296. Il nous semble que cette approche respecte la réalité familiale et le rôle que le législateur donne aux parents, lequel ne leur impose pas l'intervention de l'État si cette dernière n'est pas requise.

297. Voir la partie I :, section 2 :, sous-section B. «En vertu de la Loi sur la protection des personnes dont l'état mental présente un danger pour elles-mêmes ou pour autrui», cidessus.

298. Il n'y a pas lieu de faire de distinction entre une ordonnance de soins de santé rendue par la Cour du Québec, Chambre de la jeunesse suivant les pouvoirs qui lui sont confiés par la Loi sur la protection de la jeunesse et l'ordonnance de soins rendue par la Cour supérieure en vertu de l'article 16 C.c.Q.. La personne visée est aussi inviolable sous une loi que sous l'autre et les conditions sont les mêmes. Elles ont été énoncées par la Cour d'appel dans l'affaire G.(A.), supra note 48. 
Quant à leur approche globale face au mineur et à sa capacité de consentir, les médecins de l'Hôpital B retiendront, pour déterminer l'aptitude d'un mineur de 14 ans et plus, les mêmes critères que ceux applicables à un adulte. Ils n'inféreront pas de cette minorité une inaptitude reliée à l'âge. Pour eux, la distinction quant à l'âge est faite par la loi de sorte qu'on ne traitera pas un mineur de 14 ans et plus qui ne consent pas, à moins que son jugement ne soit altéré par la maladie. Dans ce cas, la situation sera signalée au Directeur de la protection de la jeunesse. Ainsi, dans l'hypothèse où un mineur est hautement à risque suicidaire, mais que son jugement n'est pas altéré par sa maladie, on ne le traitera pas et la tendance sera donc de respecter son refus, s'il n'y a pas de motif grave justifiant l'intervention du Directeur de la protection de la jeunesse.

Encore une fois, à notre avis, cette pratique témoigne de la mauvaise compréhension des lois. Les médecins de l'hôpital B ont raison de ne pas inférer de la minorité une inaptitude et de ne pas traiter d'emblée un mineur de 14 ans ou plus qui ne consent pas. L'article 16 C.c.Q. a été édicté pour répondre à cette situation. Ils font erreur de signaler la situation au Directeur de la protection de la jeunesse dans les cas où le jugement est altéré par la maladie. Si c'est là le seul motif du signalement, il n'a pas sa raison d'être car les parents peuvent consentir à sa place. De plus, cette approche met ces adolescents dans une situation précaire car elle préconise de respecter leur refus de traitement, s'ils sont aptes et suicidaires, les exposant ainsi à un passage à l'acte. Leur capacité à consentir aux soins est donc élargie par les intervenants qui s'inspirent mal, et à tort, du Code civil du Québec. Or, nous rappelons que la capacité du mineur de refuser des soins est limitée par la loi ${ }^{299}$. Les intervenants omettent donc de tenir compte des limites d'un tel refus reconnues par l'article 16 du Code civil du Québec et de la possibilité de recourir au tribunal. Enfin, le droit à l'autodétermination de l'enfant de 14 ans et plus prend une place démesurée et peut, dans certain cas, empiéter sur son droit d'être protégé ${ }^{300}$, tel que reconnu dans la Loi sur la protection de la jeunesse ou d'être secouru, tel que reconnu dans la Charte des doits et libertés de la personne ${ }^{301}$. Nous croyons que ces lois, comme le Code civil du Québec, permettent de protéger un jeune dangereux pour lui-même en outrepassant son refus de traitement.

299. Supra notes 103-105.

300. Girard, supra note 60 à la p. 4.

301. Supra note 41. 
Le quatrième exemple que nous souhaitons relater concerne le Centre hospitalier C, où l'on appliquera deux lois en même temps, soit la Loi sur la protection des personnes dont l'état mental présente un danger pour ellesmêmes ou pour autrui et la Loi sur la protection de la jeunesse. Le choix dépendra des particularités de chaque cas et de l'urgence de la situation. Ainsi, pour les mineurs de moins de 14 ans, on obtiendra le consentement des parents qui pourra suffire à établir et à appliquer une garde en établissement et un plan de traitement, comme à l'Hôpital B et contrairement à l'Hôpital A. Dans les cas où les parents refuseraient l'hospitalisation et où le mineur présenterait un risque pour lui-même, l'approche sera la même que celle qui sera adoptée pour un mineur de 14 ans et plus, et elle s'effectuera en deux temps.

D'abord, si la situation de l'enfant est déjà connue à la Direction de la protection de la jeunesse, on téléphonera à l'intervenant social pour l'informer de la situation et surtout pour qu'il fasse le nécessaire pour obtenir une ordonnance qui permettra de garder l'enfant, quel que soit son âge ${ }^{302}$. Le problème rencontré, cependant, est relatif aux délais car il semble que l'obtention d'une telle ordonnance prenne deux à trois jours ${ }^{303}$. Dans l'intervalle, le médecin s'autorisera des dispositions de la Loi sur la protection des personnes dont l'état mental présente un danger pour elles-mêmes ou pour autrui pour hospitaliser le mineur contre son gré, en attente de l'ordonnance de la Cour du Québec, Chambre de la jeunesse.

Si l'enfant n'est pas connu du Directeur de la protection de la jeunesse, alors on appliquera les dispositions de la Loi sur la protection des personnes dont l'état présente un danger pour elles-mêmes ou pour autrui. Un rapport psychiatrique sera préparé immédiatement et remis à l'avocat du centre hospitalier qui ira à la Cour obtenir une ordonnance de garde pour 21 jours. Cette garde laissera la latitude nécessaire aux médecins pour, d'une part,

302. Il doit être question ici de saisir la Cour du Québec, Chambre de la jeunesse, pour obtenir une ordonnance suivant L.P.J., supra note 10, art. 79 ou 76.1. Voir la partie I :, section 2 :, sous-section A. «En vertu de la Loi sur la protection de la jeunesse», ci-dessus.

303. Ce délai doit être administratif, vu L.P.J., ibid., art. 46-47 qui permettent au Directeur, en cas d'urgence, de confier un enfant sans délai et sans ordonnance à un centre hospitalier. L'article 36.2 du C.p.c. permet également à la Cour du Québec d'ordonner une garde en établissement. 
convaincre le jeune patient d'accepter d'être traité et, d'autre part, le libérer dès que la garde n'est plus requise. Cette loi est privilégiée car sous la Loi sur la protection de la jeunesse, le processus est plus lourd et moins rapide puisqu'il est requis de retourner devant le tribunal pour faire lever la garde ${ }^{304}$. De plus, on considère que cette façon de procéder respecte davantage les droits du patient puisque, dès qu'il est sous garde, il a accès à toute la documentation requise pour la contester, le cas échéant, et la faire lever. En ce qui a trait aux ordonnances de la Cour du Québec, Chambre de la jeunesse, qui confie l'enfant au centre hospitalier, contrairement à la pratique de l'Hôpital A, on ne croit pas qu'elle permette de traiter le patient, à moins que l'ordonnance ne l'autorise spécifiquement. Autrement, aucun traitement ne sera administré au patient sans son consentement, comme pour les adultes.

Nous constatons que les intervenants du Centre hospitalier $\mathrm{C}$ semblent ignorer l'existence de l'article 39 de la Loi sur la protection de la jeunesse et leur approche dépendra donc des gestes posés par ceux qui les auront précédés. Si la situation de l'enfant a déjà été signalée et évaluée, le Directeur de la protection de la jeunesse interviendra, sinon ils opteront pour une autre voie. C'est une erreur, car l'article 39 est clair et les vise spécifiquement.

D' autre part, les intervenants du Centre hospitalier C ont raison de croire que pour traiter un mineur sans son consentement, une ordonnance qui le prévoit explicitement est requise et n'est pas implicite à l'ordonnance de garde. Leur démarche serait donc complète s'ils utilisaient les dispositions législatives pour obtenir une ordonnance de traitement, soit l'article 16 C.c.Q. ou la Loi sur la protection de la jeunesse.

En somme, en aucun cas on n'utilisera les disposition de l'article 16 C.c.Q. pour saisir le tribunal et obliger un mineur de 14 ans et plus à être traité et ce, dans les trois hôpitaux que nous avons énumérés. De plus, la notion de dangerosité semble complètement ignorée. Mentionnons par ailleurs qu' aucune des approches ne tient compte de la mission spécifique des établissements concernés et que la situation est signalée au Directeur de la protection de la

304. Il est exact que pour faire lever une ordonnance rendue par la Chambre de la jeunesse à l'effet de confier l'enfant à un centre hospitalier, il est requis de retourner devant le tribunal puisque le juge doit déterminer la durée d'une telle ordonnance. 
jeunesse, sans même que les intervenants ne se soient demandés si la situation nécessitait une intervention de nature psychosociale. Dans ce cas, il s'agira d'une intervention ponctuelle et utilitaire, effectuée dans un but précis, soit l'obtention d'une ordonnance, sans complémentarité ni échange d'information.

Ajoutons enfin qu'il n'existe pas de moyen pour coordonner l'intervention médicale à l'intervention psychosociale lorsque la situation est signalée au Directeur de la protection de la jeunesse ${ }^{305}$. Ce problème de coordination est d'ailleurs à l'origine de la création du Comité sur le suicide chez les jeunes ${ }^{306}$. Ce groupe de travail avait pour but «d'étudier les modalités de mise en œuvre d'une interface entre les réseaux médical et social» ${ }^{307}$ afin «d'éliminer les obstacles empêchant la concertation entre les deux principaux réseaux d'intervention auprès des jeunes suicidaires ${ }^{308}$. Dans un communiqué de presse du 15 avril 1999, le Collège des médecins du Québec, par la voix de son président, le docteur Yves Lamontagne, indiquait qu'il fallait «promouvoir une meilleure organisation des services médicaux et psychiatriques, [...] favoriser l'arrimage de ceux-ci avec les services sociaux [et] identifier les facteurs d'entrave à la concertation des réseaux médical et social [...]» ${ }^{309}$. En ce qui a trait à l'Association des centres jeunesse du Québec, elle annonçait, dans un communiqué de presse du même jour, qu'elle travaillerait conjointement avec le Collège des médecins à la lutte au suicide chez les jeunes

305. Dans une étude du phénomène du suicide chez les adolescents en centre de réadaptation, parmi les 39 centres ayant complété le sondage dont les données couvraient les années 1986 à 1988, il a été identifié que sur les 45 centres de réadaptation au Québec, «très peu de centres ont des ententes spécifiques avec les ressources de la communauté pour intervenir dans les situations de tentatives de suicide ou de suicide. Il s'agit d'ententes avec un hôpital (7 centres), avec les services ambulanciers(3), avec les corps policiers (2) ou avec UrgenceSanté (1)», Association des centres d'accueil du Québec, supra note 8 à la p. 13.

306. L'identification de ce problème était amorcée en 1992 par l'Association des Centres d'accueil du Québec, dans son étude Le phénomène du suicide chezles adolescents en centre de réadaptation, supra note 8 à la p. 227 qui recommandait alors que chaque centre établisse des ententes de collaboration avec les centres de pédopsychiatrie ou de psychiatrie pour recevoir du support et développer son expertise.

307. Association des centres jeunesse du Québec, Collège des médecins du Québec et Protecteur du citoyen, supra note 218 à la p. 3 .

308. Ibid. à la p. 5.

309. Collège des médecins du Québec, Communiqué, «Jeunes et santé mentale : deux priorités pour le Collège des médecins du Québec» (15 avril 1999), en ligne : <http://www.cmq.org/i_pres61.htm> (Date d'accès : 26 novembre 2001). 
en centres jeunesse et que cette nouvelle collaboration levait «un des grands obstacles qui s'imposait encore» dans la lutte contre le suicide des jeunes ${ }^{310}$.

Nul doute que ce problème «d'arrimage» et de manque de concertation existe et que tous les efforts doivent être faits pour l'enrayer. Les travaux du Comité sur le suicide sont concluants à cet égard. Nous croyons cependant qu'il y a lieu d'ajouter que le problème émane également d'une mauvaise application des lois édictées par le législateur qui a bien cerné le rôle de chacun dans la gestion du risque suicidaire ${ }^{311}$. En effet, nous constatons que dans chacun des établissements consultés, une partie de l'approche adoptée est conforme aux lois en vigueur ${ }^{312}$. Cependant, il y a également un volet de leur démarche qui viole carrément sinon le texte de loi, du moins l'esprit ${ }^{313}$. Nous ne pouvons donc pas en pointer un seul en indiquant «voici ce qu'il faut faire», pas plus que nous avons trouvé un centre qui pourrait servir de modèle sur ce qu'il ne faut pas faire. Les disparités d'un établissement à l'autre témoignent de l'incompréhension des lois et de leur application. Elles démontrent également qu'il est nécessaire de tenter de rendre complémentaires les ressources médicales et sociales ${ }^{314}$. Enfin, nous concluons de ces vérifications, bien que sommaires, combinées à notre expérience dans ce domaine et à l'absence de protocoles uniformisés au sein du réseau, que l'intervention actuelle est sans

310. Association des centres jeunesse du Québec, Communiqué, «L'Association des centres jeunesse du Québec travaillera conjointement avec le Collège des médecins à la lutte au suicide chez les jeunes en centres jeunesse» (15 avril 1999), en ligne : http://www.acjq.qc.ca/04_actualite/top_01b.htm> (Date d'accès : 26 novembre 2001).

311. Voir la partie I : «La protection du mineur suicidaire : une réalité législative bien articulée», ci-dessus.

312. Voir, à titre d'exemple, les Politiques et procédures émises par l'Hôpital A en ce qui concerne les mineurs de 14 ans et plus et la pratique de l'Hôpital B en ce qui concerne les mineurs de moins de 14 ans de même que leur approche face à la capacité de consentir du mineur de 14 ans ou plus.

313. Voir, à titre d'exemple, les Politiques et procédures émises par l'hôpital A en ce qui concerne les mineurs de moins de 14 ans et la pratique de l'Hôpital B en ce qui concerne les mineurs de 14 ans ou plus de même que le fait que la Loi sur la protection des personnes présentant un danger pour elles-mêmes ou pour autrui n'est jamais utilisée. Également, constitue, à notre avis, une violation de la loi la pratique du centre hospitalier $\mathrm{C}$ qui n'applique pas L.P.J., supra note 10, art. 39. Voir également la position du Comité sur le suicide chez les jeunes, supra note 230.

314. $\quad$ Supra notes 305-308. 
encadrement et varie non seulement d'un établissement à l'autre, mais même d'un professionnel à l'autre ${ }^{315}$.

\section{B. Une analyse critique des correctifs suggérés par le milieu}

\section{i) La position du Collège des médecins}

Suite à l'adoption, par le Bureau du Collège des médecins, du rapport du groupe de travail composé de médecins psychiatres et d'omnipraticiens pour examiner l'organisation des soins en santé mentale et l'accessibilité aux soins psychiatriques au Québec ${ }^{316}$, deux médecins de famille et deux pédopsychiatres impliqués dans les soins auprès des jeunes, et membres de ce groupe de travail, se sont réunis pour élaborer des recommandations concrètes dans le but d'améliorer l'accessibilité et la qualité des services médicaux aux jeunes du Québec. En avril 1999, le Collège des médecins rendait public l'énoncé de position dans lequel le sous-groupe de travail propose des recommandations qui élaborent un système d'organisation des services médicaux en santé mentale pour les première, deuxième et troisième lignes, dans le but de mieux répondre aux besoins des jeunes ${ }^{317}$. Sans reprendre en détail le contenu des 26 recommandations, nous pouvons les résumer sous quatre volets.

Au niveau des services de première ligne, on propose que les médecins soient sensibilisés aux problématiques de santé mentale par une formation en faculté de médecine et en formation continue, que les soins aux jeunes soient considérés comme prioritaires, que les médecins généralistes soient capables de diagnostiquer, d'orienter, de traiter et de suivre les problèmes en fonction du DSM-IV, tout en étant en mesure d'évaluer les composantes sociales, familiales et environnementales des problèmes, et enfin que les infirmières soient intégrées

315. Voir, à titre d'exemple, David, supra note 19, et particulièrement, le témoignage du psychiatre.

316. Collège des médecins du Québec, «Accessibilité aux soins psychiatriques et aux services en santé mentale au Québec, supra note 215.

317. Collège des médecins du Québec, Accessibilité aux soins médicaux et psychiatriques pour la clientèle des adolescents : Énoncé de position, supra note 188. 
aux services de première ligne et favorisent la liaison entre le médecin, le patient et sa famille ${ }^{318}$.

Au niveau de la deuxième ligne de services médicaux, on propose que les généralistes ayant développé une expertise en santé mentale soient identifiés et servent de ressource, dans leur territoire respectif, auprès des autres intervenants ${ }^{319}$. Aussi, que ces médecins soient disponibles en priorité pour leurs collègues de première ligne afin de compléter l'évaluation, de diagnostiquer, traiter et suivre les jeunes en difficulté et, au besoin, d'assurer une consultation en pédopsychiatrie. On propose également que des liens étroits soient établis avec les pédopsychiatres et que l'ensemble des psychiatres soit sensibilisé à la problématique et reçoive la formation requise ${ }^{320}$. En ce qui a trait à la formation suggérée pour les intervenants de première et de deuxième ligne, le sous-groupe mentionne spécifiquement que cette formation devrait porter sur la Loi sur la protection de la jeunesse ${ }^{321}$ mais passe sous silence les articles qui concernent les mineurs dans le Code civil du Québec et la Loi sur la protection des personnes dont l'état mental présente un danger pour elles-mêmes ou pour autrui. Normalement, les psychiatres connaissent déjà ces dispositions mais, tel qu'indiqué, il semble qu'en pratique, pour un jeune suicidaire, on ignore son application ${ }^{322}$.

Au niveau de la troisième ligne de services médicaux, il est proposé de planifier et de répartir les effectifs médicaux en développant un «répertoire» des pédopsychiatres disponibles qui assureront la consultation, le soutien et la formation aux deux première lignes, dirigeront le travail clinique de l'équipe multidisciplinaire et planifieront des programmes d'intervention en santé mentale $^{323}$. Ces pédopsychiatres devraient être regroupés à l'intérieur d'un département régional afin d'assurer une cohérence dans les activités et un partage équitable des tâches ${ }^{324}$.

318. Ibid. à la p. 4.

319. Ibid.

320. Ibid. à la p. 5.

321. Ibid.

322. Voir sous la section A. «Une analyse critique du cadre d'intervention actuel», ci-dessus.

323. Collège des médecins, supra note 188 à la p. 5.

324. Ibid. à la p. 6. 
En ce qui a trait au problème particulier des jeunes pris en charge par le réseau des centres jeunesse, ce sous-groupe, après avoir reconnu que cette clientèle souffre dans une proportion inquiétante de psychopathologie et a droit à des services auxquels elle n'a pas accès, qu'il s'agit d'un problème de santé publique $^{325}$ et que les intervenants nient souvent la composante médicale des problèmes auxquels ils sont confrontés ${ }^{326}$, recommande que des services médicaux soient intégrés dans les centres jeunesse avec des services infirmiers qui sont essentiels pour assurer la liaison entre les intervenants sociaux et les médecins. Il recommande de plus que tous les jeunes signalés pour troubles de comportement et ceux pris en charge par les centres de réadaptation fassent l'objet d'un dépistage systématique et, selon le besoin, d'une évaluation médicale et d'un suivi ${ }^{327}$. À cette fin, des protocoles de dépistage des jeunes à risque devront être élaborés par les médecins qui devront ensuite procéder rapidement à l'évaluation. De plus, le sous-groupe recommande que l'intervenant social prépare un rapport écrit sur les comportements du jeune à l'attention du médecin qui aura la responsabilité d'évaluer l'enfant et, lorsque requis par le médecin, il faudra lui donner accès à l'ensemble du dossier psychosocial $^{328}$. Les intervenants concernés devront collaborer à cette évaluation et favoriser la participation de la famille. On recommande également que le médecin soit impliqué au sein des équipes jeunesse et que «les protocoles d'intervention des centres jeunesse sur la prévention du suicide soient révisés afin d'y inclure une évaluation et un suivi médical (car) il est important de reconnaître que la consultation, le transfert ou l'hospitalisation en psychiatrie est, avant tout, une décision médicale ${ }^{329}$. Des services de consultation en pédopsychiatrie devraient être disponibles pour les médecins qui œuvrent dans

325. Ibid.

326. Ibid. à la p. 7.

327. Ibid. à la p. 8.

328. Ceci est tout à fait impossible dans le cadre de la législation actuelle qui prévoit que le dossier est confidentiel. Des amendements législatifs seraient donc requis mais le Collège des médecins n'en traite pas. L'Association des centres jeunesse fait la même remarque, Association des centres jeunesse du Québec, Commentaires de l'Association des centres jeunesse du Québec sur le rapport du sous-groupe de travail sur l'accessibilité aux soins psychiatriques et aux services de santé mentale pour la clientèle jeunesse, août 1998 [non publié] à la p. 12 [ci-après Commentaires sur le rapport du sous-groupe de travail sur l'accessibilité pour la clientèle jeunesse].

329. Collège des médecins du Québec, supra note 188 à la p. 8. 
les centre jeunesse et ceux-ci devraient être associés aux plans d'intervention ${ }^{330}$. Des activités multidisciplinaires périodiques devraient être organisées pour discuter des cas complexes ${ }^{331}$.

La formulation de l'énoncé selon lequel «il est important de reconnaître que la consultation [...] en psychiatrie est [...] une décision médicale» nous laisse perplexe. S'il signifie que l'évaluation d'un risque suicidaire est un acte exclusivement médical, nous ne pouvons que féliciter le Collège de cette prise de position avec laquelle nous sommes en accord. S'il avait été affirmé clairement et plus tôt, nous croyons que la conduite des intervenants, dans le cas de Y. notamment, aurait été différente. Cependant, si cet énoncé signifie que la décision sur l'opportunité d'une consultation en psychiatrie doit être prise par un médecin, nous sommes d'avis que le Collège fait encore une erreur qui risque de perpétuer la situation actuelle. La consultation en psychiatrie par une personne suicidaire pour obtenir un diagnostic ne doit pas être précédée d'une décision médicale. Elle doit avoir lieu tout simplement. Il s'agit de l'exercice d'un droit ${ }^{332}$ et de l'exécution d'un devoir de la part des médecins ${ }^{333}$.

Étant donné que le modèle proposé est un défi important, le sous-groupe suggère de mettre en place des projets-pilotes, des projets de recherche et de développer des protocoles de dépistage et d'intervention conjointement avec les intervenants sociaux ${ }^{334}$. Le sous-groupe conclut que ce modèle devrait permettre, à court terme, d'offrir des ressources médicales aux adolescents, de briser les barrières entre les intervenants médicaux et sociaux et de favoriser une saine collaboration entre eux et, à moyen terme, de diminuer le taux de suicide chez les jeunes en outillant davantage les médecins et les intervenants ${ }^{335}$.

330. Ibid. aux pp. 8-9.

331. Ibid. à la p. 9 .

332. Ce droit est prévu dans la L.S.S.S., supra note 27, art. 5, 7; L.P.J., supra note 10, art. 8; Charte des droits et libertés de la personne, supra note 41, art 1-2.

333. Ce devoir est prévu au Code de déontologie des médecins, supra note 40, art. 2.02.01; L.P.P.D., supra note 11, art. 7. Voir nos commentaires à cet égard à la partie $\mathrm{I}$ :, section 2 :, sous-section B. au point i) «La notion de dangerosité», ci-dessus. Voir également LesageJarjoura, Lessard et Philips-Nootens, supra note 38 à la p. 199.

334. Collège des médecins du Québec, supra note 188 à la p. 10.

335. Ibid. à la p. 11. 
Quant à nous, il est hors de notre champ d'expertise de nous prononcer sur l'organisation des services médicaux que propose cet énoncé de position. Le Collège des médecins a jugé «important de faire la promotion d'une organisation de services médicaux en santé mentale répondant adéquatement aux besoins des adolescents ${ }^{336}$. Les objectifs poursuivis sont louables, certes, mais l' ampleur du projet impressionne et nous apparaît difficile à réaliser à court terme. De plus, il propose des réformes majeures sans vraiment tenir compte du système déjà en place et bien encadré par la législation actuelle et qui a certainement des forces que la société a, sans aucun doute, intérêt à exploiter. Par exemple, le sous-groupe propose d'intégrer des services médicaux aux centres jeunesse avec des soins infirmiers qui leur apparaissent essentiels pour assurer la liaison entre les intervenants sociaux et les médecins. Or, dans le réseau actuel des services sociaux, les travailleurs sociaux sont déjà des agents de liaison puisqu'ils agissent en tant que délégués du Directeur de la protection de la jeunesse et assurent la liaison entre ce dernier qui est décideur, les intervenants dans le milieu familial et les éducateurs qui agissent auprès du jeune hébergé en centre de réadaptation. Ajouter un agent de plus ne ferait, à notre avis, que diluer davantage la transmission de l'information, diminuant, par le fait même, l'efficacité de l'intervention. Bien que l'on puisse penser que l'ajout d'un agent médical ou infirmier aurait l'avantage d'intégrer cet aspect dans la pratique actuelle, ce n'est pas là l'objectif poursuivi par le Collège qui souhaite à cet égard un agent de liaison de plus. Or, nous avons vu, dans notre analyse de l'enquête du Coroner dans le cas du décès de $\mathrm{Y}^{337}$, que le nombre d'intervenants de diverses disciplines n'a pas apporté une contribution valable à la protection de la vie de Y. Il nous est apparu au contraire, que cela a nui. De plus, la Coroner a relevé des déficiences au niveau de la transmission des informations et des documents nécessaires à la continuité de la prise en charge et elle fait à cet égard, des recommandations spécifiques ${ }^{338}$. Ajouter un autre intervenant risque d'accroître cette difficulté. Nous croyons qu'il faudrait plutôt les réduire et assurer une ligne directe entre le praticien en contact avec le jeune

336. Ibid. à la p. 3 .

337. David, supra note 19.

338. Ibid. aux pp. 60, 71, 72 . 
et le médecin qui aura la charge de procéder à l'évaluation du risque suicidaire $^{339}$.

Nous devons enfin rappeler que, dans la mesure où le mineur suicidaire est en danger, l'intervention doit se faire dans un contexte d'urgence pour préserver la vie ainsi menacée. Le médecin doit évaluer sans délai l'état mental pour déterminer si le mineur est dangereux pour lui-même et, le cas échéant, il doit instaurer rapidement les mesures de protection requises ${ }^{340}$. Cette démarche est déjà bien intégrée dans notre droit et dans la pratique médicale en psychiatrie $^{341}$. Il semble toutefois que les règles sous-jacentes à cette démarche ne soient pas appliquées, dans les faits, à la situation des mineurs. Nous devons également rappeler que la première recommandation adoptée par le Bureau du Collège des médecins visait à assurer aux jeunes en situation de crise une consultation rapide ${ }^{342}$. Il nous apparait donc que la première proposition qui aurait dû être formulée par le Collège est l'élaboration de moyens pour que ces mineurs particulièrement à risque soient évalués rapidement par un médecin, comme le prévoit notre système législatif. Or, cet objectif de rapidité semble s'être perdu dans des propositions qui visent des réformes de fond ${ }^{343}$ qui ne peuvent s'actualiser à court terme, alors que les outils qui sont déjà en place dans nos lois devraient être appliqués par les intervenants. Le sous-groupe aurait donc eu intérêt à consulter et comprendre les lois en vigueur applicables aux mineurs suicidaires, qui ne sont guère différentes de celles qui s’appliquent

339. L'Association des centre jeunesse va dans le même sens que nous et fait une recommandation spécifique à cet égard, Association des centres jeunesse du Québec, supra note 328 à la p. 11 .

340. Collège des médecins du Québec, supra note 188 à la p. 6 : «Attendu que tout retard à établir un diagnostic et à prodiguer les soins médicaux requis peut aggraver la morbidité et la mortalité chez cette clientèle et qu'il s'agit là d'une situation urgente».

341. Voir la partie I :, section 2 :, sous-section B. au point i) «La notion de dangerosité», cidessus.

342. Supra note 217.

343. Ces réformes sont, entre autres, au niveau de la formation universitaire et de la formation continue, de l'élaboration des priorités des futurs départements régionaux, du développement de l'expertise des médecins de famille et la réorganisation des services qu'ils peuvent dispenser, la planification et la répartition des effectifs médicaux, l'élaboration d'un répertoire et de paramètres pour que les adolescents pris en charge par les centres jeunesse aient accès aux services médicaux, l'organisation de ces services par région, l'élaboration de grilles de dépistage et de protocoles et l'implantation d'un modèle. 
aux majeurs, pour en tenir compte dans leurs recommandations afin qu'elles ne restent pas lettre morte. Ceci aurait peut-être simplifié leur tâche.

\section{ii) La position de l'Association des centres jeunesse du Québec}

En juillet 1998, le sous-groupe de travail mis sur pied par le Collège des médecins avait soumis un premier document de travail diffusé à certains groupes pour fins de discussion. Dans ses grandes lignes, ce premier rapport s'est retrouvé, avec quelques modifications, dans le document dont nous venons de discuter. En août 1998, l'Association des centres jeunesse du Québec commentait par écrit les forces et les faiblesses de ce document et formulait ses propres recommandations ${ }^{344}$.

En premier lieu, l'Association des centres jeunesse relève les grandes forces du projet élaboré par le Collège des médecins qui reconnaît que les problèmes manifestés par les jeunes relèvent de facteurs médicaux, sociaux et environnementaux et que les intervenants ont une responsabilité partagée dans le dépistage, l'identification, le traitement, le suivi et la prise en charge des jeunes en difficulté. L'Association des centres jeunesse indique cependant, et à juste titre, que le projet du Collège des médecins ne traduit pas correctement cette perspective globale ni l'esprit de collaboration nécessaire ${ }^{345}$.

Pour l'Association, la formation professionnelle et les initiatives de recherche sont de bonnes idées ${ }^{346}$. La priorité jeunesse dans les départements régionaux constitue une initiative majeure ${ }^{347}$ et l'implication des médecins de famille dans l'aide à la jeunesse est une voie sensée eu égard au volume anticipé $^{348}$. De plus, l'Association conçoit positivement le fait que le médecin de famille soit membre d'une équipe multidisciplinaire. Elle demande toutefois la réciprocité dans la collaboration et dans l'échange de l'information par la communication du résultat de l'évaluation et la production diligente, à la

344. Commentaires sur le rapport du sous-groupe de travail sur l'accessibilité pour la clientèle jeunesse, supra note 328.

345. Ibid. à la p. 2.

346. Ibid. à la p. 4.

347. Ibid.

348. Ibid. à la p. 3. 
personne responsable au centre jeunesse, d'un rapport d'évaluation préparé par le médecin ${ }^{349}$. Par ailleurs, il est important de noter que si l'examen clinique psychiatrique permettait de poser un diagnostic et d'instaurer un traitement de nature à résoudre les difficultés du jeune, il y a tout lieu de croire que le Directeur de la protection de la jeunesse pourrait ainsi mettre fin à son intervention et déclarer que la sécurité et le développement de l'adolescent sont non compromis puisque pris en charge par le corps médical ${ }^{350}$. À titre d'exemple, prenons le cas de V., âgé de 14 ans, dont la situation a été signalée à la Direction de la protection de la jeunesse pour troubles de comportement sérieux. L'évaluation révélera que depuis son enfance, V. est impulsif, agressif, irritable, téméraire, agité et turbulent. Il présente des troubles d'apprentissage et l'école l'a menacé d'expulsion à plusieurs reprises. Il se place régulièrement en situation de danger, fait des crises de colère et, depuis 3 ou 4 ans, est obsédé par différents thèmes et est régulièrement envahi par des idées suicidaires ${ }^{351}$. La mère ne parvient pas à corriger la situation. Le signalement pourrait être retenu sous l'article $38 \mathrm{~h})^{352}$ et les mesures pourraient consister en un hébergement en centre de réadaptation. Puis V. est amené à l'urgence pour tentative de suicide par électrocution dans son bain. L'évaluation psychiatrique permet d'établir le diagnostic du Syndrome de Gilles de la Tourette avec trouble de l'attention et ainsi, d'instaurer un traitement par médication. Si les symptômes décrits cidessus sont alors résorbés, ou en voie de l'être, et que les parents assurent le suivi médical de leur enfant, le Directeur de la protection de la jeunesse devra fermer son dossier, l'usager étant pris en charge par le centre le plus apte à lui

349. Ibid. Le Collège des médecins a donné effet à cette demande dans l'une de ses recommandations. Voir Collège des médecins du Québec, supra note 188 à la p. 8. Ce rapport est déjà exigé par la Loi sur la protection des personnes dont l'état mental présente un danger pour elles-mêmes ou pour autrui. Bien que ce rapport ne soit pas destiné aux intervenants sociaux mais au juge, il pourrait néanmoins permettre au Directeur de la protection de la jeunesse de compléter son enquête sur la compromission. Il s'agirait donc, à notre avis, de coordonner l'échange d'information et non pas de créer un rapport de plus.

350. Dans la mesure où les parents collaborent à la prise en charge médicale et qu'il n'y a pas d'autres motif qui permettrait de déclarer que la sécurité ou le développement de l'enfant est compromis suivant L.P.J., supra note 10, art. 38 ou 38.1.

351. Inspiré de Maranda, supra note 1 à la p. 386.

352. De la Loi sur la protection de la jeunesse, supra note 10. 
venir en aide ${ }^{353}$. Non diagnostiqué, un tel enfant pourrait demeurer plusieurs années en centre de réadaptation et, peut-être, y mourir ${ }^{354}$.

L'Association ne tient pas compte de cette réalité lorsqu'elle soulève les aspects du projet qui requièrent des développements. À notre point de vue, c'est là que les rédacteurs de ce rapport commencent à errer à leur tour. En effet, l'Association des centres jeunesse prend pour acquis que l'intervention de la Direction de la protection de la jeunesse est requise et que tous les jeunes dont il est question sont sous sa juridiction ou devraient l'être :

[...] la très grande majorité, sinon la totalité des jeunes dont il est ici question se trouvent sous la responsabilité du DPJ. [...] De fait, les diverses directions de la protection de la jeunesse des seize centres jeunesse retiennent environ 25000 signalements par année. Ces enfants se trouvent presque tous dans des conditions extrêmement pénibles et il n'est pas surprenant que plusieurs manifestent, au moment de leur prise en charge, des troubles de la personnalité. Tous ne souffrent cependant pas de maladie mentale. Si tous sont placés

353. Voir la partie préliminaire au point ii) «Les établissements et leur mission», ci-dessus; voir également la partie I :, section 2 :, sous-section A. «En vertu de la Loi sur la protection de la jeunesse».

354. Voir David, supra note 19. Voir également le rapport d'enquête du Coroner dans le cas de E. L. décédée à l'âge de 16 ans au Centre Notre-Dame de Laval d'une encéphalopathie anoxique secondaire à la pendaison : C. Paquin, «Rapport d'investigation du Coroner A1198888» 2 juin 1998 [non publié, archivé au Bureau du Coroner à Ste-Foy]. Ce cas n'a pas fait l'objet d'une autopsie psychologique de sorte qu'il n'est pas possible de déterminer pour l'instant si une maladie mentale était à l'origine des troubles de comportements et du geste suicidaire. Il en va de même pour les cas suivants : Y. Turmel, «Rapport d'investigation du Coroner A-107464» 8 mars 1996 [non publié, archivé au Bureau du Coroner à Ste-Foy], (asphyxie par pendaison à l'âge de 14 ans au Centre jeunesse L'Escale). L. Nolet, «Rapport d'investigation du Coroner A-89739» 27 janvier 1989 [non publié, archivé au Bureau du Coroner à Ste-Foy], (asphyxie par pendaison à l'âge de 14 ans au Pavillon Richelieu). R..C. Michaud, «Rapport d'investigation du Coroner A-114470» 21 juillet 1997 [non publié, archivé au Bureau du Coroner à Ste-Foy. Dans ce dernier cas, le mineur est décédé à l'âge de 16 ans d'asphyxie par pendaison derrière la maison familiale. Il était connu de la DPJ depuis l'âge de trois ans et avait fait l'objet de plusieurs placements. L'année précédente, il avait fait une tentative de pendaison et aucune consultation en psychiatrie n'avait été demandée. Le Coroner retiendra post-mortem une dépression d'intensité moyenne et une personnalité borderline. 
sous la responsabilité du directeur de la protection de la jeunesse, tous n'ont évidement pas besoin de services médicaux spécialisés ${ }^{355}$.

Or, il n'est pas exact de prétendre que tous les jeunes dont il est question se trouvent sous la responsabilité $d u$ DPJ, tel qu'en fait foi la pratique en milieu hospitalier ${ }^{356}$. Si c'était le cas, cela ferait partie des correctifs à apporter ${ }^{357}$. L'Association semble prendre pour acquis qu'elle a juridiction sur tous les enfants en difficulté du Québec alors que ce n'est pas ce que la législation édicte $^{358}$. Par ailleurs, non seulement l'Association n'a pas une juridiction aussi étendue, mais les centres jeunesse du Québec ne disposent pas, à l'heure actuelle, «des aménagements nécessaires pour recevoir et traiter les personnes atteintes de maladie mentale» ${ }^{359}$ et suicidaires. En effet, il faut noter que l'Association, suite à ses travaux sur le suicide chez les jeunes des centres jeunesse, complétés en 1995, énonçait que l'objectif ultime était d'arriver à ce que «chaque centre jeunesse du Québec possède une politique formelle et un programme exhaustif pour prévenir les suicides et intervenir auprès des jeunes suicidaires» et que son mandat serait rempli si, d'ici quelques années, «chacun des centres de jeunesse du Québec : «[a]ppliquait un programme intégré d'activités [...] d'évaluation des situations à risque [,] d'intervention lors d'une crise suicidaire» et «[d]isposait des outils cliniques au fonctionnement efficace de ce programme $[\ldots] \gg^{360}$. Dans ces circonstances, il nous apparaît évident que ne pouvant prodiguer les services auxquels les jeunes suicidaires ont droit, les centres jeunesse ne devraient tout simplement pas les prendre en charge.

L'Association continue sur cette lancée en soumettant que le Collège doit inscrire son projet dans le cadre de la Loi sur la protection de la jeunesse ${ }^{361}$.

355. Commentaires sur le rapport du sous-groupe de travail sur l'accessibilité pour la clientèle jeunesse, supra note 328 aux pp. 5-6.

356. Voir la sous-section A. «Une analyse critique du cadre d'intervention actuel», ci-dessus. Voir également notre partie préliminaire et la partie I :, section 2 : «Les lignes d'exception», ci-dessus.

357. Voir nos commentaires à la sous-section A. «Une analyse critique du cadre d'intervention actuel», ci-dessus.

358. Ibid. Voir également Deleury et Rivet, supra note 111 aux p. 31, 34.

359. L.P.P.D., supra note 11, art. 9.

360. Voir Association des centres jeunesse du Québec, supra note 1 à la p. 7.

361. Commentaires sur le rapport du sous-groupe de travail sur l'accessibilité pour la clientèle jeunesse, supra note 328 à la p. 7. 
C'est là où le bât blesse du côté de la protection de la jeunesse. L'Association devrait savoir que le projet du Collège des médecins ne devrait pas être inscrit dans le cadre de la Loi sur la protection de la jeunesse mais dans le cadre du système législatif québécois, ce qui inclut le Code civil du Québec ${ }^{362}$ et la Loi sur la protection des personnes dont l'état mental présente un danger pour elles-mêmes ou pour autrui ${ }^{363}$. L'Association indique de plus que le cadre général et la portée du projet du Collège des médecins doivent être précisés pour le ramener dans le sillon de la Loi sur la protection de la jeunesse, que les médecins doivent étendre la pratique du signalement et intégrer cet aspect dans leur formation ${ }^{364}$. Encore une fois, la Loi sur la protection des personnes dont l'état mental présente un danger pour elles-mêmes ou pour autrui de même que les dispositions du Code civil du Québec sont passées sous silence, comme si la seule loi applicable était la Loi sur la protection de la jeunesse. Or, la pratique du signalement ne devrait pas être étendue mais plutôt appliquée conformément à cette loi. Cela ne devrait pas se traduire par une pratique plus étendue mais mieux ciblée ${ }^{365}$.

L'Association recommande qu'une mesure spéciale, instaurée de façon accélérée, permette aux intervenants d'obtenir l'expertise d'un professionnel de la santé dans les cas de tentative de suicide ou lorsque l'intervenant a des raisons de croire que l'enfant est à haut risque. Elle demande donc qu'on crée d'ici un an un groupe d'intervention ALERTE-SUICIDE ${ }^{366}$. L'Association semble ainsi ignorer que cette mesure spéciale est déjà adoptée par le législateur dans la Loi sur la protection des personnes dont l'état mental présente un danger pour elles-mêmes ou pour autrui ${ }^{367}$. Les intervenants des centres jeunesse devraient donc être informés qu'un enfant à haut risque suicidaire tombe sous le coup de cette loi qu'ils ont le devoir d'appliquer en conduisant l'enfant à l'urgence pour

362. Voir la partie I :, section 1 : «Le régime de droit commun pour les soins de santé des mineurs», ci-dessus.

363. Supra note 11. Voir également la partie I :, section 2 : «Les régimes d'exception», ci-dessus.

364. Commentaires sur le rapport du sous-groupe de travail sur l'accessibilité pour la clientèle jeunesse, supra note 328 à la p. 5.

365. Voir la partie I :, section 2 : «Les régimes d'exception», ci-dessus. Voir également dans la présente partie, la section $2:$ :La réconciliation des rôles», ci-dessous.

366. Commentaires sur le rapport du sous-groupe de travail sur l'accessibilité pour la clientèle jeunesse, supra note 328 à la p. 8.

367. Supra note 11. 
qu'il ait un examen clinique psychiatrique ${ }^{368}$. La même remarque s'impose lorsque l'Association demande que l'imputabilité soit précisée et notamment, que les départements régionaux garantissent que «les évaluations requises soient effectuées dans des délais raisonnables ou à l'intérieur des délais prévus par la loi ou ordonnées par le tribunal, notamment à l'intérieur d'un délai de trente jours lorsque la DPJ a dû appliquer des mesures d'urgence» ${ }^{369}$.

L'Association soulève, avec raison, que les parents sont les grands absents du projet du sous-groupe ${ }^{370}$. Cependant, ils sont tout aussi absents du projet de l'Association qui ne tient aucunement compte du fait que certains enfants malades peuvent être orientés dès le signalement vers le réseau de la santé par l'application des lois autres que la Loi sur la protection de la jeunesse ${ }^{371}$.

Enfin, l'Association souligne à juste titre que les infirmières ne devraient pas être agents de liaison car ce n'est certes pas en ajoutant un intermédiaire que les rapports interprofessionnels vont s'améliorer. Les intervenants doivent communiquer directement et nous sommes tout à fait en accord avec cette $\operatorname{position}^{372}$.

Ces deux rapports, pourtant élaborés par des gens du milieu certainement expérimentés dans la problématique de l'accessibilité aux services des jeunes en danger, sont un bel exemple de l'absence de communication et de la confusion qui existent dans le réseau, confusion au niveau des rôles respectifs

368. Voir la partie I :, section 2 : «Les régimes d'exception», ci-dessus.

369. Commentaires sur le rapport du sous-groupe de travail sur l'accessibilité pour la clientèle jeunesse, supra note 328 à la p. 9. Sous la Loi sur la protection des personnes dont l'état mental présente un danger pour elles-mêmes ou pour autrui, le délai est de 72 heures, il est donc encore plus rapide et les mécanismes sont déjà en place. Nul besoin de parler d'imputabilité mais de prendre les moyens pour appliquer la bonne loi. Dans un tel cas et à notre avis, l'imputabilité de l'omission reposerait sur les intervenants des centres jeunesse, voir la partie I :, section 2 :, sous-section B. «En vertu de la Loi sur la protection des personnes dont l'état mental présente un danger pour elles-mêmes ou pour autrui» et particulièrement au point i) «La notion de dangerosité».

370. Commentaires sur le rapport du sous-groupe de travail sur l'accessibilité pour la clientèle jeunesse, ibid.à la p. 9.

371. Voir L.P.J., supra note 10, art. 5, 50.

372. Voir à cet égard nos commentaires supra notes 337-339. 
et des mécanismes législatifs déjà en place qui permettent actuellement à chacun de remplir ce rôle au bénéfice de l'usager.

\section{SECTION 2 : LA RÉCONCILIATION DES RÔLES}

Notre système législatif relatif aux jeunes suicidaires comprend non seulement la Loi sur la protection de la jeunesse, mais également le Code civil du Québec, la Loi sur la protection des personnes dont l'état mental présente un danger pour elles-mêmes ou pour autrui et la Loi sur les services de santé et les services sociaux. Cette réalité est souvent complètement méconnue par les personnes qui ont à prendre des décisions relativement à l'accessibilité de ces jeunes aux soins psychiatriques. Pour protéger les mineurs suicidaires, nous pensons que tous les intervenants œuvrant en établissement et ayant à prendre des décisions à leur sujet doivent connaître ce système législatif et lui donner effet. Pour ce faire, ces intervenants doivent en premier lieu comprendre la mission propre à l'établissement dans lequel ils œuvrent, apprendre à jouer le rôle qui est le leur suivant la loi et enfin, faire intervenir l'État ou les tribunaux uniquement lorsque nécessaire.

\section{A. Réapprendre sa mission}

La Loi sur les services de santé et les services sociaux énonce les objectifs fondamentaux du régime, les droits des usagers et les règles relatives à l'accessibilité aux services. L'organisation des établissements de santé et de services sociaux est prévue, leur rôle et divers pouvoirs et devoirs leur permettant d'assurer la prestation des services y est précisée. Dans cette loi, le réseau est conçu comme un tout et l'usager est rattaché à ce tout, plutôt qu'à un établissement en particulier et ce, quelle que soit la porte à laquelle il frappera en premier lieu.

\section{i) Évaluer les besoins et orienter les personnes}

Tel qu'indiqué, la situation d'un adolescent suicidaire peut être signalée, évaluée et retenue sous plusieurs alinéas des articles 38 et 38.1 de la Loi sur la 
protection de la jeunesse. De façon générale cependant, ce sera sous l'alinéa h) de l'article $38^{373}$ que le Directeur de la protection de la jeunesse sera le plus souvent appelé à intervenir, c'est-à-dire dans les cas où l'adolescent «manifeste des troubles de comportement sérieux et que ses parents ne prennent pas les moyens nécessaires pour mettre fin à la situation (...) ou n'y parviennent pas».

L'évaluation se fera à partir des faits signalés et des données recueillies lors du traitement du signalement et la personne autorisée par le Directeur de la protection de la jeunesse rassemblera des informations portant sur les aspects suivants :

* la nature exacte des faits signalés,

* la nature exacte de faits autres que ceux signalés pertinents aux diverses situations visées par les articles 38 et 38.1 de la L.P.J.,

* les impacts de ces faits sur l'enfant,

* les capacités des parents,

* les conditions de vie de l'enfant ${ }^{374}$.

On peut affirmer sans risque de se tromper qu'un jeune qui pose des gestes suicidaires manifeste des troubles de comportement sérieux. Il en va de même pour un jeune qui s'infligerait des blessures ou encore qui serait agressif envers autrui. Toutefois, la seule présence de troubles de comportement chez un enfant n'entraîne pas l'application du paragraphe h) de l'article 38 de la Loi sur la protection de la jeunesse. Cet article énonce deux conditions.

D'abord, ces troubles doivent être «sérieux» et seront jugés tels «en fonction de critères de permanence, de répétition, d'étendue, d'intensité, de fréquence, de violence et de danger.» ${ }^{375}$ L'usage abusif de drogue ou d'alcool, l'agressivité physique ou verbale, la tentative de suicide ou l'automutilation, que ces causes soient reliées ou non à des causes d'ordre psychologiques, constituent

373. Déjà cité, sous la partie I :, section 2 :, sous-section A. «En vertu de la Loi sur la protection de la jeunesse», ci-dessus.

374. Ministère de la Santé et des Services sociaux, Manuel de référence sur la Loi sur la protection de la jeunesse, Direction du Ministère de la Santé et des Services sociaux, Québec, 1990 à la p. 65.

375. Ibid. à la p. 48. 
de tels troubles de comportement ${ }^{376} \mathrm{~s}^{\prime}$ ils sont bien ancrés dans la dynamique du jeune :

Pour justifier une intervention, il faut apprécier l'ensemble des réactions de l'enfant. Ne sont pas visés par la loi les comportements qui ne sont pas la manifestation d'un type de comportement acquis ou en voie de le devenir de façon certaine et inéluctable, surtout s'ils sont causés par des situations transitoires, sinon provoquées par les adultes $^{377}$.

Mentionnons qu'il n'est pas nécessaire que le jeune soit passé à l'acte. Les verbalisations d'agressivité envers lui-même ou envers autrui constituent des manifestations au sens de cet article qui peuvent permettre de conclure que l'enfant est en situation de danger et qu'il a besoin de protection. Si les verbalisations sont plus de l'ordre de la manipulation que de l'ordre des tendances suicidaires, sa sécurité ou son développement pourront également être déclarés compromis. Dans un tel cas cependant, les mesures qui seraient privilégiées pour corriger la situation seraient de l'ordre de mesures de rééducation et non des soins de santé, si la deuxième condition de l'article 38 est remplie. Pour en arriver à ce résultat, la maladie mentale doit être éliminée, ce que seul un médecin peut faire ${ }^{378}$.

La seconde condition pour que l'article $38 \mathrm{~h}$ ) trouve application est le cas où les parents du jeune sont incapables d'assurer sa sécurité, de le convaincre de consulter un professionnel, ou d'accepter une hospitalisation pour être traité ${ }^{379}$. Dans de telles situations, ils seraient alors incapables de prendre les moyens nécessaires pour mettre fin à la situation qui compromet la sécurité ou le développement de leur enfant :

376. Boulais, supra note 137 à la p. 182.

377. Ibid. aux pp. 181-182.

378. Loi médicale, supra note 29. Voir également supra notes 181-182 de même que Collège des médecins du Québec, supra note 188 aux pp. 6-7.

379. Ces deux dernières situations ne visent que le mineur de 14 ans et plus car dans le cas d'un mineur de moins de 14 ans, ce sont les parents qui consentent aux soins de santé pour leur enfant. Voir la partie I :, section 1 :, sous-section A. «Le consentement substitué ou le consentement personnel du mineur», ci-dessus. 
Les parents ne prennent pas les moyens nécessaires soit parce qu'ils ne le veulent pas ou qu'ils ne le peuvent. On fait référence dans le premier cas à leur volonté d'exercer leur responsabilité parentale face à la situation de l'enfant ou encore à leur reconnaissance des troubles de comportement chez leur enfant. Dans le deuxième cas il s'agit de leur capacité parentale qui est en cause.

Les parents ne parviennent pas à corriger la situation soit à cause de leurs limites personnelles (déficience parentale à assumer le contrôle de l'enfant, permissivité, inconsistance) ou à cause du choix inapproprié [sic] des moyens en regard de l'état de l'enfant ou encore de l'inaccessibilité et de l'insuffisance de ces moyens ${ }^{380}$.

Si les deux conditions prévues à l'article 38 h) sont présentes, la sécurité ou le développement de l'enfant sera alors déclaré compromis pour ce motif. L'intervention du Directeur de la protection de la jeunesse est donc exclue si son évaluation permet de conclure que les parents prennent les moyens nécessaires pour corriger la situation ${ }^{381}$. Dans une décision rendue en 1989, la Cour d'appel a eu l'occasion d'interpréter, dans le cadre de l'ancienne formulation de l'article $38 \mathrm{~g}$ ), le sens des mots «avoir pris les moyens nécessaires pour corriger la situation». La Cour du Québec et la Cour supérieure en appel avaient refusé la requête du Directeur de la protection de la jeunesse. Ce dernier demandait que la sécurité et le développement de l'enfant soient déclarés compromis en raison de l'abus sexuel commis par le mari de sa mère. Cet abus était admis par la mère, son conjoint et l'enfant, mais ils contestaient une déclaration de compromission en alléguant que la mère avait pris les moyens pour corriger la situation, tel qu'indiqué dans l'article $38 \mathrm{~g}$ ). Sur cette question, la Cour d'appel s'exprime ainsi :

Or, deux conditions sont posées par cet alinéa pour que la situation prévue au paragraphe $\mathrm{g}$ ) ne puisse donner lieu à une déclaration au sens de l'article 38 :

380. Association des centres de services sociaux, Les enfants en besoin de protection, août 1984 [non publié] aux pp. 25-26 cité dans Manuel de référence sur la Loi sur la protection de la jeunesse, supra note 374 à la p. 48.

381. Protection de la jeunesse-228, [1986] R.J.Q. 2906. (C.Q.) 
1. $[\ldots]$

2. que les parents aient pris les dispositions nécessaires pour corriger la situation.

[...] H.L. me semble avoir adopté l'attitude d'un parent responsable :

1) Dès le jour où elle a appris les abus sexuels, elle a signalé la situation au directeur de la Protection de la jeunesse.

2) Elle a pris les moyens physiques pour éviter d'autres répétitions de la situation.

3) Elle a favorisé une thérapie familiale et y a participé activement ${ }^{382}$.

La Cour d'appel a donc conclu que, comme le parent avait pris les moyens nécessaires pour corriger la situation, la sécurité ou le développement de l'enfant ne pouvait faire l'objet d'une déclaration de compromission en vertu de l'article 38. Ce cas, appliqué aux faits qui nous concernent, signifie que l'enfant suicidaire ne doit être pris en charge par le Directeur de la protection de la jeunesse que si les parents ne prennent pas les moyens pour corriger la situation ou n'y parviennent pas. Prendre les moyens peut signifier consulter à l'urgence d'un hôpital. S'ils l'ignorent, c'est la tâche des intervenants de les informer ${ }^{383}$ et de les orienter vers cette ressource.

Ainsi, si les parents ont la capacité de prendre les moyens pour corriger la situation, le signalement ne doit pas être retenu et le Directeur de la protection de la jeunesse n'a pas juridiction. Dans un tel cas, il doit informer «l'enfant et ses parents des ressources disponibles dans leur milieu ainsi que des modalités d'accès à ces services et à ces ressources ${ }^{384}$ et il peut, s'ils y consentent, les diriger vers «les établissements, les organismes ou les personnes les plus aptes à leur venir en aide» ${ }^{385}$. C'est ainsi que devraient s'ouvrir les portes des établissements de la santé pour les enfants suicidaires qui ont des parents en

382. Protection de la jeunesse-423, [1989] R.J.Q. 2663. (C.A.).

383. L.P.J., supra note 10, art. 50.

384. Ibid.

385. Ibid. Voir également L.S.S.S.S., supra note 27, art. 82 au même effet. 
mesure de s'en occuper. De la même façon, si ces troubles de comportement sont la manifestation d'une maladie mentale, c'est vers une ressource médicale que les interventions doivent en premier lieu être dirigées.

\section{ii) Le dépistage précoce}

Étant donné que le problème du suicide chez les jeunes est mixte ${ }^{386}$, une partie de la solution réside, à notre avis, dans le dépistage précoce par une consultation médicale lorsque requise suivant la législation québécoise en vigueur, afin de diriger l'enfant en détresse vers l'établissement le plus apte à lui venir en aide. Une telle consultation est obligatoire lorsque l'adolescent présente un danger pour lui-même ${ }^{387}$. Les verbalisations et les gestes suicidaires constituent d'évidence des facteurs de dangerosité ${ }^{388}$.

Tel qu'indiqué, l'évaluation d'un risque suicidaire est un acte médical qui conduit à des ordonnances de surveillance précises suivant le résultat de l'évaluation. Selon la Loi sur la protection de la jeunesse qui les habilite ${ }^{389}$, les intervenants qui agissent à titre de délégués du Directeur de la protection de la jeunesse n'ont aucune compétence médicale et ne peuvent donc pas poser de diagnostic psychiatrique. Ils ont aussi le devoir, après évaluation, de diriger le mineur dont la problématique ne relève pas de leur champ d'expertise spécifique vers les personnes, les centres ou les organismes les plus aptes à leur venir en aide. Dans la mesure où le problème est mixte, ils ont le devoir de faire le nécessaire pour que les besoins de santé soient évalués et pris en charge ${ }^{390}$.

Par ailleurs, les centres hospitaliers ne doivent pas faire appel au centre de la protection de l'enfance et de la jeunesse ni au Directeur de la protection de la jeunesse si, au préalable, une problématique de nature médicale qui serait à l'origine des difficultés que présente le jeune qui lui est référé, n'a pas été éliminée. Les troubles de comportement peuvent cacher une maladie qui

386. Voir partie préliminaire, ci-dessus et Collège des médecins du Québec, supra note 188 à la p. 3.

387. Supra notes 181-182, 332-333.

388. Supra note 166.

389. Supra note 10, art. 32-33; L.S.S.S.S., supra note 27 art. 82.

390. L.S.S.S.S., ibid., art. 81-82. 
peut conduire à des conduites dangereuses. Si c'est le cas, c'est le centre hospitalier qui est le plus apte à venir en aide au jeune.

En somme, quel que soit l'intervenant qui reçoit le jeune dans le réseau des services de santé et des services sociaux, nous avons vu que la Loi sur les services de santé et les services sociaux lui impose trois fonctions bien précises soit évaluer, dispenser et orienter vers la ressource la plus apte à lui venir en aide. Cette orientation vers une autre ressource signifie donc une évaluation clinique préalable par l'intervenant, qui déterminera que l'établissement dans lequel il œuvre n'est pas la ressource la plus appropriée. Il faut donc éviter les approches systématisées qui occultent le jugement clinique des professionnels. Le protocole adopté par l'hôpital A et commenté à la section 1 de la deuxième partie constitue un tel exemple d'une approche systématique. Les signalements automatiques à la Direction de la protection de la jeunesse en sont d'autres. Cette évaluation clinique préalable à la réorientation de l'usager vers une autre ressource doit également tenter de déterminer le champ d'expertise concerné. $\mathrm{Si}$ les besoins de ce dernier requièrent une évaluation médicale ou une garde en établissement, c'est a priori en milieu hospitalier que le réseau pourra répondre à ces besoins.

\section{iii) La complémentarité à l'intérieur du réseau}

Une lecture complémentaire des articles 81 et 82 de la Loi sur les services de santé et les services sociaux ${ }^{391}$ mène au constat que les centres de protection de l'enfance et de la jeunesse ne doivent pas garder sans évaluation ni supervision médicale des adolescents dont l'état nécessite des soins médicaux. C'est le cas, à notre avis, des adolescents suicidaires. L'inverse est aussi vrai. Les centres hospitaliers doivent signaler l'enfant qui manifeste des troubles de comportement sérieux, qui ne sont pas causés par la maladie, au 
Directeur de la protection de la jeunesse qui procédera à l'évaluation de la situation.

Ainsi, les intervenants du réseau de la santé ont, à l'égard de leurs patients mineurs, des responsabilités qu'ils ne devraient pas déléguer au Directeur de la protection de la jeunesse. Par ailleurs, ils ne devraient pas s'ingérer dans une problématique qui ne relève pas de leur champ d'expertise et diriger les mineurs, le cas échéant et après évaluation, vers les centres, les organismes ou les personnes les plus aptes à leur venir en aide. Avant que cette réorientation ne soit effectuée, les intervenants doivent éliminer toute problématique de nature médicale et tout élément de dangerosité. Dans les cas où ces éléments sont présents mais où la situation, tout en nécessitant une prise en charge, ne requiert pas d'hospitalisation, une collaboration, un suivi et une prise en charge conjointe doivent être assurés avant le congé.

\section{B. L'intervention étatique en dernier recours}

Nous croyons donc que les dispositions de la Loi sur la protection de la jeunesse doivent s'appliquer de façon complémentaire ou concurrente, selon les besoins du jeune suicidaire, à celles de la Loi sur la protection des personnes dont l'état mental présente un danger pour elles-mêmes ou pour autrui et du Code civil du Québec. En droit, nous n'avons aucune difficulté à concilier ces trois avenues juridiques disponibles au Québec pour tenter de protéger les mineurs suicidaires. Elles visent des objectifs analogues, la protection des personnes vulnérables, avec des moyens qui ne sont pas contradictoires. En fait, les voies sont complémentaires dans la mesure où elles sont bien comprises et donc bien appliquées. Pour ce faire, l'intervenant du réseau qui reçoit le jeune doit, en premier lieu, cerner la nature de ses besoins et déterminer ensuite si l'intervention du titulaire de l'autorité parentale ou du tuteur pourra être suffisante pour qu'il reçoive une réponse adéquate à ses besoins. Ce n'est que dans la négative qu'il y a lieu de déterminer si l'intervention de tiers détenant un pouvoir décisionnel est requise. Dans un tel cas, la problématique en cause permettra d'identifier le tiers en question, soit le Directeur de la protection de la jeunesse, un juge de la Cour supérieure qui agira suivant les pouvoirs qui lui sont conférés par l'article 16 C.c.Q. ou un juge de la Cour du Québec qui agira suivant la Loi sur la protection des personnes dont l'état mental présente un danger pour elles-mêmes ou pour autrui. 


\section{i) L'intervention du DPJ comme mesure d'exception}

L'intervention en protection de la jeunesse doit se limiter aux situations dans lesquelles la sécurité ou le développement est compromis. On doit se garder d'intervenir dans des situations où la loi ne s'applique pas et ce, même s'il existe des besoins réels chez l'enfant ou sa famille. Ces besoins pourront trouver des réponses auprès d'autres organismes sociaux ou communautaires ou dans les établissements énumérés dans la Loi sur les services de santé et les services sociaux, tels les centres locaux de services communautaires ou les centres hospitaliers, sans qu'il soit requis de mettre en œuvre tout le mécanisme de la Loi sur la protection de la jeunesse. Le législateur n'a pas voulu, en donnant ce nom à cette loi, donner au Directeur de la protection de la jeunesse une juridiction sur tous les enfants du Québec ni un droit de regard sur toutes les familles en général et les attitudes parentales en particulier ${ }^{392}$. Le législateur souhaite simplement qu'on vienne en aide aux enfants en besoin de protection. Rien de plus ni rien de moins.

\section{- L'obligation de signaler}

Tel qu'indiqué ${ }^{393}$, tout le mécanisme de protection de l'enfant est mis en œuvre par le biais d'un signalement transmis au Directeur de la protection de la jeunesse par toute personne qui a un motif raisonnable de croire que la sécurité ou le développement d'un enfant est compromis. L'article 39 de la Loi sur la protection de la jeunesse impose l'obligation de signaler aux professionnels qui, par la nature même de leur profession, prodiguent des soins ou toute autre forme d'assistance à des enfants et qui, dans l'exercice de leur profession, ont un motif raisonnable de croire que la sécurité ou le développement est ou peut être considéré comme compromis au sens de l'article 38 ou au sens de l'article 38.1 .

392. Malgré l'opinion de l'Association des centres jeunesse qui semble à l'effet contraire énoncée supra note 355, Association des centres jeunesse du Québec, supra note 328 aux p. 5-6. Voir Deleury et Rivet, supra note 111 à la p. 31 et s.

393. Voir la partie I :, section 2 :, sous-section A. «En vertu de la Loi sur la protection de la jeunesse», ci-dessus. 
Le législateur voulait ainsi s'assurer que les professionnels qui entrent en contact avec les enfants, en raison de leur fonction, signalent les situations qui sont portées à leur connaissance si ces situations sont de nature à mettre les enfants en danger au sens large. Cette obligation incombe à tous les employés des établissements, tels que définis dans la Loi sur les services de santé et les services sociaux ${ }^{394}$. À l'article 94 de cette loi, l'établissement est celui qui exerce une activité propre à la mission d'un centre local de services communautaires, d'un centre hospitalier, d'un centre de la protection de l'enfance et de la jeunesse, d'un centre d'hébergement de longue durée ou d'un centre de réadaptation.

Le texte de loi impose l'obligation légale de dénoncer au Directeur la situation et ce, malgré le devoir de respecter le secret professionnel, sauf en ce qui concerne l'avocat. Cet article est donc dérogatoire à l' article 9 de la Charte des droits et libertés de la personne ${ }^{395}$.

L'article 39 de la Loi sur la protection de la jeunesse indique que «la personne doit avoir un motif raisonnable de croire» : la teneur de l'obligation variera donc suivant le jugement que la personne portera sur les circonstances. Étant cependant une obligation légale, tout doute sur l'opportunité ou non de signaler devrait se résoudre en faveur de l'enfant et de sa protection. Il ne revient pas à l'auteur du signalement de déterminer si, de fait, l'enfant est ou non en situation de compromission. Dans l'affaire Commission scolaire Baldwin-Cartier c. Commission de protection des droits de la jeunesse ${ }^{396}$ la Cour supérieure devait statuer sur l'étendue du devoir d'un directeur d'école de signaler une situation prévue à l'article $38 \mathrm{~g}$ ) (abus sexuels). Ce directeur avait été informé par la mère d'un des élèves de l'école de la possibilité que des enfants d'une garderie, située à proximité de l'école, soient victimes d'abus sexuels. Ce directeur n'avait pas jugé à propos de signaler la situation et une plainte pour absence de signalement avait été portée contre lui. Cette plainte était contestée par la commission scolaire qui était d'avis que le directeur d'école n'avait pas de motif raisonnable pour agir. Le Tribunal ne partagea pas cet avis et indiqua que l'article 39 crée une obligation d'ordre général et que si

394. Supra note 27.

395. Supra note 41.

396. (7 décembre 1990), Montréal 500-05-011419-908, J.E. 91-338 (C.S.). 
la notion de motif raisonnable crée un doute dans l'esprit de celui qui est informé de faits troublants, il ne doit pas être l'arbitre du caractère raisonnable : il doit signaler.

Ainsi, pour les intervenants du réseau qui ont à intervenir auprès des enfants, il est important de bien comprendre que ce n'est pas le rôle de la personne qui fait le signalement de prouver et de convaincre que l'enfant est en situation de compromission. La conclusion à l'effet que l'enfant est en situation de compromission est une décision qui relève des pouvoirs exclusifs du Directeur de la protection de la jeunesse et qui sera prise après son enquête sur la situation de l'enfant ${ }^{397}$. C'est lui qui, par la suite, aura le fardeau de prouver devant le tribunal, le cas échéant, que l'enfant est en situation de compromission. L'auteur du signalement ne serait, dans ce contexte qu'un témoin des faits qui l'ont amené à avoir un motif raisonnable de croire que la sécurité ou le développement de l'enfant était compromis.

Enfin, l'opportunité ou non de signaler pourra également découler de la problématique du mineur et des besoins qu'il présente en gardant à l'esprit la mission propre à chacun des établissements ${ }^{398}$. Si les besoins du mineur sont de nature exclusivement médicale et non de nature psychosociale, il n'y aura probablement pas lieu de signaler la situation mais plutôt d'utiliser d'autres dispositions de la loi pour répondre aux besoins concernés, notamment le Code civil du Québec et la Loi sur la protection des personnes dont l'état mental présente un danger pour elles-mêmes ou pour autrui ${ }^{399}$.

\section{- La problématique de nature psychosociale}

Les manquements du ou des parents à l'égard des soins de santé requis par l'état de leur enfant qui serait dangereux pour lui-même peuvent se qualifier sous plusieurs alinéas de l'article 38 notamment b), c), e) et h) et sous l'article

397. L.P.J., supra note 10 , art. 32, 49.

398. Partie préliminaire, ci-dessus.

399. Par exemple, voir le cas de V. relaté dans Maranda, supra note 1 à la p. 386; partie II :, section 1 :, sous-section A. au point ii) «La pratique en vase clos», ci-dessus. 
38.1 c) de la Loi sur la protection de la jeunesse déjà cités ${ }^{400}$. Cependant, étant donné que «la responsabilité d'assurer le soin, l'entretien et l'éducation de l'enfant et d'en assurer la surveillance incombe en premier lieu à ses parents» ${ }^{401}$, il faut, pour retenir un signalement, être strictement dans le cadre de ces articles. Les motifs de compromission énumérés dans la loi sont limitatifs. Étant une loi d'exception, elle doit recevoir une interprétation restrictive :

L'autorité parentale demeure la règle, l'intervention sociale ou judiciaire, l'exception ${ }^{402}$.

Il est reconnu que la Loi sur la protection de la jeunesse n'a pas été conçue pour répondre à tous les besoins ${ }^{403}$ et ne modifie en rien le fondement juridique de la responsabilité parentale énoncé au Code civil du Québec, en vertu duquel les parents ont toujours été considérés comme les premiers responsables de leur enfant. Dans la décision Protection de la jeunesse-228, l'honorable juge André Sirois cite le rapport de la Commission parlementaire spéciale sur la protection de la jeunesse, rendu le 23 novembre 1982 (rapport Charbonneau ), qui exprime, selon lui, fort adéquatement la philosophie sousjacente à la loi :

Il arrive des situations particulières qui commandent que la responsabilité parentale soit assistée, corrigée ou assumée temporairement par l'État. Ce sont de telles situations que définit, précise et circonscrit la Loi sur la protection de la jeunesse.

Il est important de rappeler que la Loi sur la protection de la jeunesse ne modifie en rien le fondement juridique de la responsabilité

400. Voir la partie I :, section 2 :, sous-section A. «En vertu de la Loi sur la protection de la jeunesse», ci-dessus.

401. L.P.J., supra note 10, art. 2.2.

402. (18 avril 1980), Montréal 500-41-003430-791 (Trib. jeun.), juge Lamarche. Néanmoins, il est important de souligner que la loi doit s'interpréter à la lumière de l'intérêt de l'enfant, notion très large prévue à l'article 3 qui donne au juge un pouvoir discrétionnaire. Une jurisprudence abondante de la Cour suprême cerne l'interprétation de cette notion et en confirme la constitutionnalité. Voir notamment Young c. Young, [1993] 4 R.C.S. 3; P.(D.) c. S. (C.), [1993] 4 R.C.S. 141 et C.M. c. Catholic Children's Aid Society of Metropolitan Toronto, supra note 124.

403. Boulais, supra note 137 à la p. 151. 
parentale et que l'intervention des services publics dans la vie privée des familles ne peut se fonder que sur un motif raisonnable de croire que la sécurité et le développement d'un enfant sont compromis. Les parents se voient alors reconnu le droit à des services d'aide qui ne tentent qu'à rétablir, supporter, restaurer ou renforcer leur capacité de parent.

$[\ldots]$

C'est donc en tout dernier recours, dans la situation où l'action et la présence même des parents [sont jugées contraires] à l'intérêt de l'enfant et au respect de ses droits, que la loi peut signifier pour les parents le terme de leur prérogative parentale.

Une véritable protection de l'enfant consiste d'abord à la sauvegarde de ses droits, dans la satisfaction de ses besoins en considération de son meilleur intérêt. À ce titre, la protection est assumée en premier lieu par les parents, ensuite la communauté d'appartenance et enfin l'État qui peut aider, supporter, ou, exceptionnellement se substituer aux parents lorsqu'ils ne peuvent ou ne veulent pas assumer leur responsabilitée ${ }^{404}$.

\section{ii) L'intervention du tribunal si nécessaire}

L'intervention étatique peut prendre la forme judiciaire. Avant d'y avoir recours, il nous apparaît essentiel d'insister sur l'importance de ne recourir au tribunal qu'en cas de nécessité et pour les bons motifs. Il faut également choisir la bonne voie législative car cette voie aura un impact sur la durée de l'hébergement, sur la rapidité du diagnostic et, dans certains cas, son existence $^{405}$.

404. Protection de la jeunesse-228, supra note 381 à la p. 2909.

405. David, supra note 19. Voir également les rapports de Paquin, Turmel, Nolet et Nolet, supra note 354. Voir enfin Gagné, supra note 6 à la p. 377. 
La requête pour examen clinique psychiatrique ${ }^{406}$ est comparable à la requête pour hébergement obligatoire provisoire ${ }^{407}$. Des mesures de protection peuvent cependant être instaurées avant l'audition de telles requêtes et ce, sous le régime mis en place par les deux lois. Alors que sous la Loi sur la protection des personnes dont l'état mental présente un danger pour elles-mêmes ou pour autrui, le danger doit être grave et immédiat, pour donner ouverture à une garde sans ordonnance, laquelle ne pourra excéder 72 heures, sous le régime de la Loi sur la protection de la jeunesse, le retrait du milieu sans ordonnance judiciaire peut être prolongé de 24 heures à 7 jours, si cela est nécessaire et si le maintien dans le milieu risque de causer un dommage sérieux. Le critère est moindre et les délais sont plus longs.

L'ordonnance de garde en établissement prendra fin sans autre formalité par le seul écoulement du temps ou si un certificat délivré par le médecin traitant atteste qu'elle n'est plus justifiée. Ce n'est pas le cas de l'hébergement obligatoire qui ne prendra fin avant son expiration que sur ordonnance du tribunal. En principe, la durée totale d'un hébergement obligatoire est de 30 jours. Ce délai est de rigueur et peut être renouvelé une seule fois pour 30 jours. Cependant, des facteurs tels l'encombrement des rôles des tribunaux combinés aux disponibilités des procureurs et les délais nécessaires pour compléter les évaluations et les expertises rendent souvent illusoires ces délais. En pratique, les parties consentiront fréquemment à la prolongation de l'hébergement au delà du délai fixé par la loi.

Une requête pour hébergement provisoire est un incident important de l'instance en cours et son sort déterminera souvent les mesures qui seront ordonnées par le tribunal au terme de l'enquête sur la compromission. L'hébergement provisoire prendra fin à l'issue de l'enquête sur la compromission lorsque l'ordonnance sur les mesures applicables sera rendue, laquelle, dans les cas de troubles de comportement sérieux, conduira souvent à

406. Demandée en vertu de la Loi sur la protection des personnes dont l'état mental présente un danger pour elles-mêmes ou pour autrui, supra note 11 et des art. 26-31 C.c.Q. Voir la partie I :, section 2 :, sous-section B. «En vertu de la Loi sur la protection des personnes dont l'état mental présente un danger pour elles-mêmes ou pour autrui», ci-dessus.

407. Demandée en vertu de la L.P.J., supra note 10, art. 76.1, 79. Voir également le partie I :, section 2 :, sous-section A. «En vertu de la Loi sur la protection de la jeunesse», ci-dessus. 
un hébergement en centre de réadaptation pour une durée d'une année. Cette période sera susceptible de prolongations jusqu'à la majoritét ${ }^{408}$ et, dans certains cas, rares cependant, au delà de la majoritét ${ }^{409}$.

On voit donc que l'adolescent porteur d'une maladie mentale, qui se manifesterait par des troubles de comportement sérieux et qui serait orienté en protection de la jeunesse puis en centre de réadaptation pour rééducation par le tribunal, pourrait y demeurer plusieurs mois voire plusieurs années s'il n'est pas diagnostiqué. Dans certains cas, il pourrait même s'y suicider ${ }^{410}$. Il apparaît donc de première importance de faire appel au tribunal uniquement lorsque c'est nécessaire et dans ce cas, de choisir la voie judiciaire appropriée à la problématique réelle du mineur qui en sera l'objet.

\section{- $\quad$ Pour les mineurs de moins de 14 ans}

Tel qu'indiqué, le consentement aux soins de santé est donné par le titulaire de l'autorité parentale ou par le tuteur ${ }^{411}$. L'autorisation des parents ou du tuteur suffit donc pour lui prodiguer des traitements ${ }^{412}$. L'autorisation du tribunal ne sera requise qu'en cas d'empêchement ou de refus injustifié du parent ou du tuteur et ce, suivant l'article 16 du Code civil du Québec.

L'autorisation des parents ou du tuteur est également suffisante pour garder en établissement le mineur suicidaire de moins de 14 ans sans qu'il soit nécessaire de faire intervenir le Directeur de la protection de la jeunesse ni le tribunal et ce, que le mineur soit d'accord ou non avec la mesure proposée ${ }^{413}$.

\footnotetext{
408. L.P.J., supra note 10, art. 95.

409. Ibid., art. 64.

410. Supra note 354.

411. $\quad$ Art. 14 al. 1 C.c.Q., supra note 58.

412. Voir la partie I :, section 1 :, sous-section A. «Le consentement substitué ou le consentement personnel du mineur», ci-dessus.

413. Art. 26 C.c.Q.
} 
L'autorisation du tribunal pour garder ce mineur contre son gré ne sera requise qu'en cas d'absence de consentement du parent ou du tuteur ${ }^{414}$. En principe, cette autorisation pourrait être obtenue par le biais de l'article $27 \mathrm{du}$ Code civil du Québec à la demande d'un médecin ou d'une personne intéressée qui aurait des motifs sérieux de croire que le mineur présente un danger pour luimême ou pour autrui ${ }^{415}$. Si le mineur présente un danger grave et immédiat, il doit être confié sans délai à un centre hospitalier qui pourra le garder de façon préventive jusqu'à ce que la Cour du Québec ait ordonné une garde provisoire pour que le mineur soit soumis à un examen clinique psychiatrique, le tout suivant le deuxième alinéa de l'article 27 C.c.Q. et les dispositions de la Loi sur la protection des personnes dont l'état mental présente un danger pour ellesmêmes ou pour autrui ${ }^{416}$.

Si le mineur de moins de 14 ans n'est pas pourvu d'un tuteur et s'il n'y a personne qui agit à titre de titulaire de l'autorité parentale, il y a là un motif évident pour signaler la situation au Directeur de la protection de la jeunesse puisque l'enfant serait en situation d'abandon ${ }^{417}$. S'il est ainsi pourvu, le responsable devrait prendre les mesures qui s'imposent sans nécessité d'une ordonnance judiciaire. S'il ne le fait pas, un signalement pourrait également être requis si les conditions de l'article 39 sont présentes ${ }^{418}$.

Qu'il y ait ou non signalement, il nous apparaît que la Loi sur la protection des personnes dont l'état mental présente un danger pour ellesmêmes ou pour autrui et les articles 26 à 31 du Code civil du Québec doivent être appliqués dès que le mineur présente un danger pour lui-même.

414. Art. 27 C.c.Q. Voir la partie I :, section 2 :, sous-section B. «En vertu de la Loi sur la protection des personnes dont l'état mental présente un danger pour elles-mêmes ou pour autrui», ci-dessus.

415. Ibid.

416. Voir la sous-section B., ibid.

417. L.P.J., supra note 10, art. 38 b). Voir la partie I :, section 2 :, sous-section A. «En vertu de la Loi sur la protection de la jeunesse», ci-dessus.

418. Voir la sous-section A, ibid. Voir également la partie II :, section 2 :, sous-section B. «L'intervention étatique en dernier recours», ci-dessus. 


\section{- $\quad$ Pour le mineur de 14 ans et plus}

Contrairement au mineur de moins de 14 ans, l'autorisation des parents ou du tuteur ne suffit pas pour prodiguer des traitements au mineur suicidaire de 14 ans et plus apte et qui refuse de donner son consentement ${ }^{419}$. Dans le cas où les soins de santé sont requis par son état, ou dans le cas d'empêchement ou de refus injustifié du titulaire de l'autorité parentale, c'est par le biais de l'article 16 C.c.Q. que ces soins pourront être prodigués suite à l'intervention du tribunal $^{420}$.

Par contre, tout comme pour le mineur de moins de 14 ans, le seul consentement du titulaire de l'autorité parentale suffit pour garder en établissement le mineur de 14 ans et plus qui présente un danger pour luimême ${ }^{421}$. Si le titulaire de l'autorité parentale ne donne pas ce consentement, le médecin ou un intéressé qui a des motifs sérieux de croire que le mineur présente un danger en raison de son état mental, pourra demander au tribunal qu'il soit gardé en établissement provisoirement pour y subir une évaluation psychiatrique $^{422}$. En cas d'urgence, c'est-à-dire s'il présente un danger grave et immédiat en raison de son état mental, il doit être confié sans délai à un centre hospitalier qui pourra le garder de façon préventive ${ }^{423}$ jusqu'à ce que le titulaire de l'autorité parentale ait été consulté et, à défaut de consentement, jusqu'à ce que la Cour du Québec ait ordonné une garde provisoire pour qu'il soit soumis à un examen clinique psychiatrique ${ }^{424}$.

419. Voir la partie I :, section 1 :, sous-section B. au point i) «L'intervention judiciaire», cidessus.

420. Ibid., voir également Girard, supra note 60 à la p. 182.

421. Art. 26 C.c.Q.

422. Art. 27 al.1 C.c.Q.

423. Art. 27 al.2 C.c.Q.

424. Voir la partie I :, section 2 :, sous-section B. «En vertu de la Loi sur la protection des personnes dont l'état mental présente un danger pour elles-mêmes ou pour autrui». 
Dans ces cas, ce mineur n'est pas nécessairement en situation de compromission. Tel qu'indiqué, un signalement ne sera requis que si les conditions de l'article 39 sont remplies ${ }^{425}$.

\section{CONCLUSION}

Lorsque nous avons opté pour ce sujet, notre hypothèse de départ, non formulée, était que le traitement juridique du mineur suicidaire différait du traitement du majeur dans la même situation. Nous souhaitions tenter de déterminer si la Loi sur la protection de la jeunesse, dans sa formulation ou son application, pouvait être à l'origine d'une telle différence. Nous avons donc analysé l'approche adoptée par les milieux social et médical, tenté de cerner si les mineurs suicidaires étaient lésés et, le cas échéant, sous quels aspects, afin d'être en mesure de faire ressortir les failles des trois avenues juridiques pouvant être empruntées au Québec pour les protéger, pour finalement élaborer des recommandations.

L'analyse des textes de lois ne permet pas, selon nous, d'affirmer que les droits des enfants ou des adolescents, pris en charge en vertu de la Loi sur la protection de la jeunesse, sont plus ou moins bien protégés que sous les autres régimes législatifs ni que ceux des adultes qui font l'objet de mesures qui leur sont imposées en vertu de la Loi sur la protection des personnes dont l'état mental présente un danger pour elles-mêmes ou pour autrui, bien au contraire. Le fait qu'ils bénéficient presque systématiquement de l'assistance d'un avocat dans le premier cas leur offre une garantie supplémentaire à cet égard ${ }^{426}$. De plus, nous n'avons aucune difficulté à concilier, en théorie, les trois avenues juridiques disponibles au Québec pour tenter de protéger les mineurs ayant des problèmes de santé mentale de nature à mettre leur sécurité en danger et refusant les traitements qui leur sont proposés. Elles visent des objectifs analogues, la protection des personnes vulnérables, avec des moyens qui ne sont pas contradictoires. En fait, ces voies sont complémentaires dans la mesure où elles sont bien comprises et donc, bien appliquées.

425. Voir la partie I :, section 2 :, sous-section A. «En vertu de la Loi sur la protection de la jeunesse», ci-dessus. Voir également la partie II :, section 2 :, sous-section B. L'intervention étatique en dernier recours», ci-dessus.

426. L.P.J., supra note 10, art. 10. 
Par contre, il y a lieu de constater, sur le terrain, que les mineurs suicidaires ne sont pas bien orientés dans le réseau des services et que la pratique actuelle est, en réalité, en marge de la loi. Nous savons d'expérience, et espérons l'avoir démontré, que les législations pertinentes sont mal comprises et mal appliquées. Ceci se traduit par des protocoles d'intervention et des documents de formation qui sont mal conçus de sorte qu'en pratique, les jeunes suicidaires, particulièrement ceux qui sont pris en charge par les centres jeunesse, n'ont pas accès à un médecin. Ces jeunes sont évidemment des personnes au sens de nos législations et ont les mêmes droits que les adultes. Or, «toute personne a le droit d'être informée de l'existence des services et des ressources disponibles [...] en matière de services de santé et de services sociaux ainsi que des modalités d'accès ${ }^{427}$, a le droit de recevoir ces services ${ }^{428}$ et, lorsque sa vie ou son intégrité est menacée, «a le droit de recevoir les soins que requiert son état» ${ }^{429}$. Ceci signifie qu'un mineur suicidaire a le droit d'exiger qu'un diagnostic de sa condition soit posé et qu'on lui assure une surveillance proportionnelle au risque qu'il présente. Les protocoles d'intervention que nous avons étudiés dans les pages qui précèdent de même que les documents de formation adressés aux intervenants des centres jeunesse ne permettent pas l'exercice de ces droits par les jeunes de sorte que, sous le couvert de la protection de la jeunesse, ces jeunes déjà en danger voient leur situation s'aggraver car ils ne sont ni diagnostiqués ni traités adéquatement. Bien plus, le risque suicidaire est évalué par des personnes qui n'ont pas la compétence requise et la formation qu'elles reçoivent, de même que les protocoles qui encadrent leurs interventions, sont en marge de la loi.

Nous avons déjà indiqué que l'étude effectuée par le docteur Pierre Gagné et publiée en 1994, démontre l'incidence élevée de troubles mentaux chez les suicidés adolescents et adolescentes, qui serait de l'ordre de 90\%. Dans ce contexte, il nous apparaît prioritaire d'identifier rapidement dans quelle catégorie se situe l'adolescent en détresse, soit celle de la maladie mentale ou celle des problèmes psychosociaux, et ce, par une évaluation minutieuse en

427. L.S.S.S.S., supra note 27 , art. 4.

428. Ibid., art. 5 .

429. Ibid., art. 7. 
évitant les approches stéréotypées qui occultent le jugement clinique des professionnels concernés.

Pour ce faire, il faut en premier lieu cerner la nature des besoins du mineur et déterminer si l'intervention du titulaire de l'autorité parentale ou du tuteur pourra être suffisante pour que le mineur ait une réponse adéquate à ses besoins. Ce n'est que dans la négative qu'il y a lieu de déterminer si l'intervention de tiers détenant un pouvoir décisionnel est requise. Dans un tel cas, la problématique en cause permettra d'identifier le tiers en question, soit le Directeur de la protection de la jeunesse, soit un juge de la Cour supérieure qui agirait suivant les pouvoirs qui lui sont conférés par l'article 16 C.c.Q. ou un juge de la Cour du Québec qui agirait suivant la Loi sur la protection des personnes dont l'état mental présente un danger pour elles-mêmes ou pour autrui.

Les dispositions de cette dernière loi sont assez simples à appliquer en ce qui concerne les mineurs de moins de 14 ans puisque toutes les décisions relatives aux soins de santé, incluant la garde en établissement, sont prises par le titulaire de l'autorité parentale ou le tuteur en lieu et place de l'enfant. Pour les mineurs de 14 ans et plus, la situation est moins simple en raison de leur capacité d'exercice qui est «modulée» pour les questions relatives aux soins. Néanmoins, nous croyons que le législateur a fait certains choix au nom de la protection de ce dernier en permettant qu'il soit traité et même gardé contre son gré et ce, même s'il est apte à prendre une décision. Ceci n'est pas possible dans le régime de droit applicable aux majeurs, car la seule exception législative à l'autonomie et à l'inviolabilité de la personne apte est la garde en établissement. Dès que le majeur ne présente plus de danger pour lui-même ou pour autrui, la garde doit être levée même si, par ailleurs, il a besoin de soins. S'il ne consent pas à ces soins et est apte, on ne pourra pas les lui imposer. Nous croyons que le mineur de 14 ans et plus ne possède pas une autonomie aussi grande quant à sa santé ${ }^{430}$ et le triple régime qui lui est applicable en témoigne. Ce sont là des choix d'une société parfois interventionniste, il faut bien le reconnaître, mais qui peuvent se comprendre dans un système qui cherche à protéger une personne qui se trouve dans un âge critique et difficile à traverser, l'adolescence. 
Cependant, les intervenants du réseau doivent éviter de s'ingérer dans la vie privée des jeunes au-delà de ce que permet le législateur en leur imposant l'intervention de tiers, que ce soit des juges ou le Directeur de la protection de la jeunesse, dans les cas qui ne sont pas prévus par la loi. Ces intervenants doivent également chercher à rendre complémentaires les nombreuses ressources du réseau.

Pour ce faire, il nous apparaît important d'élaborer des protocoles clairs qui coordonneraient les interventions des établissements du réseau de la santé et ceux des services sociaux et qui permettraient de répondre aux problèmes identifiés dans la Partie II. Les grandes avenues de ces protocoles devraient inclure notamment les éléments essentiels suivants :

- L'évaluation d'un risque suicidaire est un acte médical qui doit donc exclusivement être posé par un médecin.

- Les personnes autorisées à consentir aux soins, à l'hospitalisation ou à une garde en établissement pour le mineur sont les titulaires de l'autorité parentale ou les tuteurs.

- L'autorisation du tribunal n'est pas requise pour administrer des soins, hospitaliser ou garder en établissement un mineur de moins de 14 ans: le consentement du titulaire de l'autorité parentale ou du tuteur suffit.

- L'autorisation du tribunal n'est pas requise non plus pour garder en établissement un mineur suicidaire de 14 ans et plus: le consentement du titulaire de l'autorité parentale ou du tuteur suffit ${ }^{431}$.

- $\quad$ Si le mineur de 14 ans et plus, apte, a besoin de soins et les refuse, il faut alors s'adresser au tribunal en vertu de l'article 16 C.c.Q. qui devrait recevoir application. Si ce mineur est, au contraire, inapte à prendre une décision, l'autorité parentale ou celle du tuteur reprend sa place.

- $\quad$ En cas d'absence, d'inaction ou de refus injustifié du titulaire de l'autorité parentale ou du tuteur à l'égard du traitement ou de la garde en établissement, il faut s'adresser au tribunal.

431. En effet, nous estimons qu'il en est ainsi même si le mineur s'y oppose, en raison de la formulation de l'article 26 al.2 C.c.Q. Supra note 88 et la partie I :, section 2 :, sous-section B. «En vertu de la Loi sur la protection des personnes dont l'état mental présente un danger pour elles-mêmes ou pour autrui», ci-dessus.. 
- $\quad$ Si le mineur présente un danger pour lui-même ou pour autrui, il doit être gardé en établissement. En l'absence de son consentement ou d'un consentement substitué, la Loi sur la protection des personnes dont l'état mental présente un danger pour elles-mêmes ou pour autrui doit s'appliquer comme pour un adulte et ce, quel que soit son âge.

- $\quad$ Toute situation d'urgence physique, mentale ou psychologique (alors que la vie est en danger ou l'intégrité menacée), lorsque le danger est grave et immédiat, permet de procéder sans consentement ni autorisation du tribunal, à des soins et/ou à la garde en établissement en vertu des articles 13 et 27 al. 2 C.c.Q.. La situation doit être régularisée par la suite selon les dispositions législatives spécifiques.

- $\quad$ On ne doit signaler à la Direction de la protection de la jeunesse que les cas où une personne a un «motif raisonnable de croire que la sécurité ou le développement est compromis», au sens de la Loi sur la protection de la jeunesse, quel que soit l'âge du mineur.

- $\quad$ Toute personne professionnelle qui prodigue des soins ou autre forme d'assistance à un mineur et qui a un «motif raisonnable de croire que sa sécurité ou son développement est compromis», a l'obligation légale de signaler la situation au Directeur de la protection de la jeunesse et ce, même si elle est liée par le secret professionnel.

- Un signalement à la Direction de la protection de la jeunesse n'est requis que dans les cas où il semble que le titulaire de l'autorité parentale ou le tuteur ne fait pas le nécessaire pour que le mineur reçoive les soins appropriés.

- Un signalement à la Direction de la protection de la jeunesse est également requis s'il apparaît que la situation nécessite des services psychosociaux que l'enfant de 14 ans et plus ou sa famille refuse.

Nous rappelons enfin que de 1980 à 1996, l'augmentation du taux de suicide des jeunes québécois âgés de 10 à 19 ans est passé du simple au double $^{432}$. Nous croyons fermement que notre société dispose des moyens efficaces pour intervenir rapidement auprès des jeunes à risque. Nous devons cependant faire en sorte qu'ils soient appliqués sans tarder. Le docteur Pierre

432. Association des centres jeunesse du Québec, Collège des médecins du Québec et Protecteur du citoyen, supra note 218 à la p. 7. 
Gagné indique que les parents, les éducateurs, et les professionnels de la santé doivent s'ouvrir à la réalité de la maladie mentale chez l'adolescent. Nous adhérons à ses propos lorsqu'il énonce: «[c]'est en identifiant les individus à haut risque et leur problématique réelle et en intervenant de façon rapide et avec des moyens efficaces qu'on peut espérer voir diminuer le nombre d'adolescents qui se suicident chaque année au Québec» ${ }^{433}$. Nous ajoutons que les intervenants doivent, en plus, s'ouvrir à la réalité juridique qui leur permettra d'atteindre cet objectif.

433. Gagné, supra note 6 à la p. 378. 